\title{
AN ALGEBRAIC STUDY OF MULTIVARIABLE INTEGRATION AND LINEAR SUBSTITUTION
}

\author{
MARKUS ROSENKRANZ, XING GAO, AND LI GUO
}

\section{Contents}

1. Introduction 1

1.1. Motivation 1

1.2. Structure of the Paper 4

1.3. Notation 5

2. Rota-Baxter Hierarchies 6

2.1. Background to the Concept of Rota-Baxter Hierarchy 6

2.2. Examples and Properties of Rota-Baxter Hierarchies 12

3. Rota-Baxter Bialgebras 19

3.1. Conceptualizing Linear Substitutions via Scaled Bialgebras 19

3.2. Integration in Scaled Bialgebras 23

4. The Ring of Partial Integral Operators 30

4.1. Crucial Operator Relations 30

4.2. Construction via Quotient 36

5. Conclusion 40

Acknowledgments $\quad 41$

$\begin{array}{ll}\text { References } & 41\end{array}$

\begin{abstract}
We set up an algebraic theory of multivariable integration, based on a hierarchy of Rota-Baxter operators and an action of the matrix monoid as linear substitutions. Given a suitable coefficient domain with a bialgebra structure, this allows us to build an operator ring that acts naturally on the given Rota-Baxter hierarchy. We conjecture that the operator relations are a noncommutative Gröbner basis for the ideal they generate.
\end{abstract}

\section{INTRODUCTION}

1.1. Motivation. The notion of integral operator plays a fundamental role in Analysis, Physics and Stochastics. Apart from some early beginnings, the systematic study of integral operators and integral equations started in earnest by the end of the nineteenth century, at the hands of towering figures like Hilbert, Fredholm and Volterra.

Despite their indubitable origin in Analysis, it is often profitable to study integral operators in an algebraic setting, i.e. to model them by the crucial notion of Rota-Baxter

Date: July 30, 2018.

MCS: 47G10，16W99，16T10，68W30; 12H05，47G20，13N10，16W70 
algebras [17]. The situation is similar to the case of differential algebra, defined to be an (associative) algebra equipped with a derivation. Its study originated from the algebraic study of differential equations by Ritt [31,32] starting in the 1930s and has since developed into a vast area of mathematics research with broad applications, including the mechanical proof of geometric theorems [25, 44]. An algebraic structure that encodes integration first appeared as a special case of a Rota-Baxter algebra, defined to be an algebra $R$ equipped with a linear operator $P$ on $R$ satisfying the Rota-Baxter axiom

$$
P(u) P(v)=P(u P(v))+P(P(u) v)+\lambda P(u v)
$$

for all $u, v \in R$. Here $\lambda \in R$ is a prefixed constant called the weight, which is zero in the case $P=\int_{0}^{x}$ but nonzero for its discrete analog (partial summation). Let us now name a few important areas where this approach proved to be successful:

- The original formulation of Rota-Baxter algebra (first called Baxter algebras) was in the context of fluctuation theory, when Glen Baxter gave a new proof [4] of the so-called Spitzer identity, based on the Rota-Baxter axiom. This line of research continues to this day; see the references $[12,61,121,163,174]$ in the survey monograph [17].

- The use of Rota-Baxter algebra for problems in combinatorics has been firmly established and widely popularized [38, 39] by Gian-Carlo Rota (which is why they are partly named after him). For a typical example, see [17, §3.3] and the references therein.

- One of the main applications of Rota-Baxter algebras is the operator form of the socalled classical Yang-Baxter equation, named after the physicists Chen-Ning Yang and Rodney Baxter (not Glen Baxter). For an overview and current developments in this area, see the references [14, 26, 46, 127, 164] in [17].

- Another important application in Physics is in the renormalization of perturbative quantum field theory, in particular the algebraic Birkhoff decomposition established by Connes and Kreimer [11]. A survey of current literature in this domain is given in $[17, \S 2.5]$.

- The relation of Rota-Baxter algebras and multiple zeta values and their generalizations is a fascinating topic [20, 21, 45]. For more on this topic, the reader may read $[17, \S 3.2]$ and the literature survey in [17, §3.4].

- Algorithmic tools for solving and factoring linear boundary problems [13, 40] are developed [33, 35, 37] based on integro-differential algebras (Rota-Baxter algebras with a suitable differential structure). The solution of the boundary problem is denoted by the Green's operator, an element in a ring of integral operators.

In this paper, we shall employ Rota-Baxter algebras for creating an algebraic theory of multivariable integation under the action of linear substitutions. As far as we are aware, this is the first time that integral operators and substitutions are studied from a systematic algebraic perspective. 
Our original motivation comes from the last of the application areas listed above: One may build up an abstract theory of linear boundary problems and their Green's operators [30], "abstract" in the sense that it abstracts from the ring-theoretic structures (derivations, Rota-Baxter operators, characters), retaining only their linear skeleton. The advantage of the abstract theory is that it encompasses both linear ordinary differential equations (LODEs) and linear partial differential equations (LPDEs), both scalar and vector problems, both continuos (differential) and discrete (difference) equations. However, unlike the integro-differential setting of [35], it does not-and cannot-provide algorithmic tools.

For developing an algebraic theory of boundary problems for LPDEs, along with the required algorithmic tools, it is first of all necessary to develop a suitable ring of partial integro-differential operators capable of expressing the Green's operators of (some of) these boundary problems. An adhoc example of such an operator ring - together with applications to solving certain simple LPDE boundary problems - can be found in [34].

It must be emphasized that a suitable ring of partial integro-differential operators is not just the tensor product of the corresponding ordinary integro-differential operator rings. Such a ring would be too weak for expressing the Green's operators of even very simple LPDEs like the inhomogeneous wave equation $u_{t t}-u_{x x}=f$ mentioned in [30, §7], and the problems treated in [34]. The reason for this is evident from a geometric perspective: The tensor product provides only axis-parallel integrators while most Green's operators require integration over oblique domains (slanted lines, triangular areas, skewed volumes). This contrasts with differential operators, where any directional derivative can be representedvia the chain rule - as a linear combination of partial ("axis-parallel") derivatives.

For rectifying this defect, it is sufficient to add linear substitutions to the operator ring. Their algebraic interation with the axis-parallel integrators is regulated by (a special case of) the substitution rule of integration. The precise algebraic formulation turns out to be surprisingly delicate, and the current axiomatization (Definition 2.3) should be seen as a first attempt in this direction. Our central question can be stated thus: "What is the algebraic structure of linear substitutions under partial integral operators?" See the Conclusion for some further ideas on how to improve and generalize the current setup.

The presence of linear substitutions also makes the choice of the coefficient algebra much more delicate (see the beginning of Section 3 for a short explanation). To incorporate closure under linear substitutions into the algebraic setting, we require the coefficients to form a Hopf algebra with a suitably compatible Rota-Baxter operator; we call the resulting structure a Rota-Baxter bialgebra (Definition 3.5).

For simplifying the further treatment, we have decided to keep differential operators out of the picture. In other words, we are building up rings of partial integral operators (with linear substitutions and coefficient functions), rather than partial integro-differential operators. In view of the applications for boundary problems, this is clearly not sufficient but it provides a crucial intermediate stage. In fact, the adjunction of partial differential operators (coming from derivations of the base algebra) leads to new operator relations that are much simpler than the ones treated here: Loosely speaking, differential operators "march through" to the far right (unlike integral operators) since the chain rule leads to a full decoupling, as indicated by our earlier remarks above. We shall develop the 
resulting operator ring in a subsequent paper, along with its application to expressing Green's operators of LPDE boundary problems.

Apart from this deliberate restriction, the operator rings constructed in this paper are a vast generalization of the adhoc ring created in $[34,36]$ for the purpose of illustrating certain techniques for solving LPDE boundary problems. To wit, the current ring is not based on the specific model of multivariate analytic functions as in [34], and it allows for a wide class of coefficient algebras (arbitrary Rota-Baxter bialgebras) rather than just constant coefficients.

From the viewpoint of Analysis, one may also pose the following question: Let $R$ be the subring of the ring of linear operators on the complex function space $C^{\infty}\left(\mathbb{R}^{n}\right)$ generated by the partial integral operators, the multiplication operators induced by certain coefficient functions, and all linear substitutions. If $\Gamma$ denotes the set of all these generators, there is an evident map $\mathbb{C}\langle\Gamma\rangle \rightarrow R$ that "evaluates" the indeterminates by the corresponding generators. The kernel $\mathcal{R}$ of this map encodes the ideal of operator relations satisfied in Analysis. If these are known, one has a full algebraization in the sense that $R \cong \mathbb{C}\langle\Gamma\rangle / \mathcal{R}$. While we have not reached this goal in the present paper, the relations $\mathcal{R}^{\prime}$ used in our formulation of the operator ring (Definition 4.8) are clearly an important subset of $\mathcal{R}$, and there are good reasons to conjecture that actually $\mathcal{R}=\mathcal{R}^{\prime}$. However, this will presumably need an analytic proof that has to be approached elsewhere.

1.2. Structure of the Paper. In Section 2 we introduce the main structures needed for the algebraic description of multivariable integration and linear substitutions. After providing some motivation, we start in Subsection 2.1 with an axiomatization of function algebras over a field $K$ with a contravariant action of the matrix monoid $\left(\mathcal{M}_{n}(K), \cdot\right)$. These structures, subsequently named K-hierarchies (Definition 2.1), serve as the basic building blocks for the partial integral operator rings - the latter act on $K$-hierarchies, and they take their coefficients from among them. Every $K$-hierarchy induces a lattice of subalgebras, which provide an algebraic formulation for functions depending on certain prescribed variables. In Subsection 2.2 we add a suitable collection of Rota-Baxter operators to a $K$-hierarchy to arrive at the pivotal notion of a Rota-Baxter hierarchy (Definition 2.3). Its core ingredient is an algebraic formulation of those cases of the substitution rule of integration that are needed for obtaining normal forms in the operator ring. The basic properties of Rota-Baxter hierarchies are then developed (Lemmas 2.7, 2.8, 2.9). The central example of a Rota-Baxter hierarchy is the collection of all smooth multivariate functions (Example 2.5).

The introduction of coefficients is studied in Section 3. Ignoring first the Rota-Baxter structure, we start in Subsection 3.1 by describing an algebraic structure that captures "separated" multivariate functions under linear substitutions. While the coproduct of the bialgebra provides the basic linear substitution $x \mapsto x+y$, proper linear combinations like $x \mapsto x+\lambda y$ can only be achieved via an additional "scaling" structure, thus leading to the notion of scaled bialgebra (Definition 3.1). Intuitively, the elements in such a scaled bialgebra are the univariate functions from which one builds all available 
coefficient functions via linear substitutions. This is made precise by the induced hierarchy (Definition 3.2), which is indeed a $K$-hierarchy (Proposition 3.3), and of course the smooth algebra - along with certain subalgebras - provides the prototypical model for this situation (Example 3.4). Next we combine scaled bialgebras with Rota-Baxter operators (Subsection 3.2). This requires a certain compatibility relation, which is in fact a special case of the substitution rule (Definition 3.5); the induced hierarchy of such a Rota-Baxter bialgebra is then a Rota-Baxter hierarchy (Theorem 3.7), and this holds in particular for the smooth algebra (Example 3.8). They form the appropriate coefficient algebras for the operator ring (Definition 3.9).

Section 4 is devoted to the construction of the operator ring, focusing first on the identification and verification of fundamental operator relations (Subsection 4.1). From the perspective of rewriting, the effect of these relations can be described intuitively as follows: In the first step (Proposition 4.2), one normalizes one-dimensional integrators - meaning integral operators with subsequent linear substitution and coefficient-by ensuring that their associated linear substitution is "minimal" (for integration along the $x_{i}$-axis: identity matrix except beneath the $i$-th diagonal element). Then the normalized one-dimensional integrators are put in ascending order (Proposition 4.4), and repetitions are eliminated (Proposition 4.7). The monomials in the resulting normal form will therefore consist of a strictly ascending list of one-dimensional integrators, as will be shown later (Theorem 4.11). In the final Subsection 4.2 we define the desired ring of partial integral operators and linear substitutions (Definition 4.8). By its very defintion, it is clear that it has a natural action on the given Rota-Baxter hierarchy (Proposition 4.9). The corresponding rewrite system is shown to be Noetherian (Theorem 4.10), meaning the computation of normal forms will always terminate. We conclude by conjecturing that the rewrite system is moreover confluent (Conjecture 4.12), meaning the normal forms are in fact unique.

In the Conclusion we provide some pointers to possible future work. On the one hand, we propose some thoughts on the next obvious steps towards building up an algebraic and algorithmic theory of LPDE boundary problems. On the more speculative side, we discuss also some ideas about potential generalizations and extensions of our theory.

1.3. Notation. The set $\mathbb{N}$ of natural numbers is assumed to include 0 . If $X$ is any set such that $0 \in X$, we write $X^{\times}=\{x \in X \mid x \neq 0\}$ for its nonzero elements.

The vector space of $m \times n$ matrices over a field $K$ is denoted by $K_{n}^{m}$, where $m=1$ or $n=1$ is omitted. The unit vectors of $K^{n}$ or $K_{n}$ are written as $e_{i}$; it will be clear from the context what $n$ is, and whether rows or columns are intended. We denote the corresponding matrix rings by $\mathcal{M}_{n}(K)=K_{n}^{n}$. The $n \times n$ identity matrix is denoted by $I_{n}$. We write $e_{i j} \in \mathcal{M}_{n}(K)$ for the matrix units, with the only nonzero entry 1 in row $i$ and column $j$. Similarly, $d_{i, \lambda}=I_{n}+(\lambda-1) e_{i i}$ denotes the scaling matrix, which is equal to $I_{n}$ except that its $i$-th diagonal entry is $\lambda$. The vertical composition of two matrices $M \in K_{n}^{r}, N \in K_{n}^{s}$ is denoted by $M \oplus N \in K_{n}^{r+s}$.

Given a $K$-algebra $\mathcal{F}$, we write $\operatorname{AlgHom}_{K}(\mathcal{F})$ for the monoid of $K$-algebra endomorphisms on $\mathcal{F}$. The opposite of a monoid or algebra $\mathcal{F}$ is denoted by $\mathcal{F}^{*}$. If $S$ is a semigroup, we use the notation $K[S]$ to refer to the semigroup algebra over $K$. In this 
paper, all (co)algebras are assumed to be (co)commutative, so in particular bialgebras and Hopf algebras are both commutative and cocommutative. In contract, the word ring shall designate unital rings that are not necessarily commutative. We apply the usual notation $[S, T]=S T-T S$ for the commutator of ring elements $S$ and $T$.

In this paper, all Rota-Baxter operators and derivations are of weight zero. Since we think of them as inspired from analysis, we write them as $\int$ and $\partial$. These symbols (along with their embellished variants) will always be used in operator notatation. For example, we write $\left(\partial_{x} f\right)\left(\int^{y} g\right)$ rather than $\partial_{x}(f) \int^{y}(g)$. Products - usually denoted by juxtapositionhave precedence over operators, so $\int^{y} f g$ is to be parsed as $\int^{y}(f g)$ and $\int^{y} f \int^{y} g$ as $\int^{y}\left(f \int^{y} g\right)$. Note that derivations like $\partial_{x}$ are indexed below to indicate their origin from $\frac{\partial}{\partial x}$; in contrast, integrators like $\int^{y}$ are indexed above as a reminder of $\int_{0}^{y}$.

In the sequel, we will use the standard variables $x_{1}, x_{2}, x_{3}, \ldots$ for defining functions of arbitrarily (but finitely) many variables. So a definition like $f\left(x_{2}\right):=x_{2}$ should be carefully distinguished from $f\left(x_{1}\right):=x_{1}$ since the first denotes the $x_{2}$-projection $\left(x_{1}, x_{2}, \ldots\right) \mapsto x_{2}$ but the second the $x_{1}$-projection $\left(x_{1}, x_{2}, \ldots\right) \mapsto x_{1}$. Sometimes it will be convenient to use the abbreviations $x \equiv x_{1}, y \equiv x_{2}, z \equiv x_{3}$. Since we do not use $x$ as a shorthand for the sequence $\left(x_{1}, x_{2}, x_{3}, \ldots\right)$, this will create no confusion. In the scope of this paper we will only deal with linear substitutions, hence we will usually drop the qualification "linear".

\section{Rota-Baxter Hierarchies}

We start by building up the basic operational domains - the algebras which the prospective ring of partial integro-differential operators is to operate on. Such domains will be called Rota-Baxter hierarchies since they encode a notion of multivariable integration in conjunction with an action of the corresponding substitution monoid.

2.1. Background to the Concept of Rota-Baxter Hierarchy. As a motivation to the general definition, let us first look at the classical setting of multivariate smooth functions. In the latter case, we are thinking of functions $f: \mathbb{R}^{n} \rightarrow \mathbb{R}$, for any arity $n \geq 0$, with the nullary ones denoting constants. To simplify the book-keeping we pass to the direct limit

$$
C^{\infty}\left(\mathbb{R}^{\infty}\right):=\bigcup_{n \geq 0} C^{\infty}\left(\mathbb{R}^{n}\right)
$$

of functions depending on arbitrarily (but finitely) many real variables.

Thus we think of $\mathcal{F}:=C^{\infty}\left(\mathbb{R}^{\infty}\right)$ as an algebra with the ascending filtration $\mathcal{F}_{n} \subset \mathcal{F}_{n+1}$ given by $\mathcal{F}_{n}=C^{\infty}\left(\mathbb{R}^{n}\right)$, so we can set up the action of arbitrary-sized real matrices. An ascending sequence of algebras $\left(\mathcal{F}_{n}\right)$ will be called an ascending algebra.

For the rest of this section, let $K$ be a field of characteristic zero so that $\mathbb{Q} \subseteq K$. We write $\mathcal{M}(K)$ for the monoid of all $\infty \times \infty$ matrices $M: \mathbb{N} \times \mathbb{N} \rightarrow K$ that can be written in the form $M=I+\tilde{M}$, where $I$ is the $\infty \times \infty$ identity matrix and where $\tilde{M}$ is any row and column finite matrix (meaning all rows except finitely many and all columns except finitely many are zero). We call $\mathcal{M}(K)$ the (linear) substitution monoid over $K$. Note that 
it has the natural ascending filtration

$$
\mathcal{M}(K)=\bigcup_{n \geq 1}\left(I+\mathcal{M}_{n}(K)\right)
$$

where we use the embedding $\mathcal{M}_{n}(K) \hookrightarrow \mathcal{M}_{n+1}(K)$ that sends $M$ to $\left(\begin{array}{cc}M & 0 \\ 0 & 1\end{array}\right)$. We will also identify finite matrices $M \in \mathcal{M}_{n}(K)$ with their embedding $\left(\begin{array}{cc}M & 0 \\ 0 & I\end{array}\right) \in \mathcal{M}(K)$. In particular, we regard scalars $\lambda \in K$ as $\lambda e_{11}+e_{22}+\cdots \in \mathcal{M}(K)$ rather than $\lambda I$. In the sequel, we will also need the descending chain

$$
\begin{aligned}
\mathcal{M}(K) & =\tilde{\mathcal{M}}_{0}(K) \supset \tilde{\mathcal{M}}_{1}(K) \supset \tilde{\mathcal{M}}_{2}(K) \supset \cdots, \\
\tilde{\mathcal{M}}_{n}(K) & =\left\{\left(\begin{array}{cc}
I_{n} & 0 \\
0 & M
\end{array}\right) \mid M \in \mathcal{M}(K)\right\} \subseteq K[\mathcal{M}]
\end{aligned}
$$

is the subring of matrices acting trivially on $K^{n}$. A rectangular matrix $M \in K_{s}^{r}$ with $r<s$ is identified with the corresponding square matrix $\tilde{M} \in K_{s}^{s}$ obtained from $M$ by adding the unit vectors $e_{r+1}, \ldots, e_{s}$ as additional rows. On the other hand, given $M \in K_{s}^{r}$ with $r>s$, we identify $M$ with $\tilde{M} \in K_{r}^{r}$ by adding zero columns. In particular, any row $v \in K_{n}$ may be viewed as a matrix $v \oplus e_{2} \oplus \cdots \oplus e_{n} \in K_{n}^{n}$, and adjoining a column vector $w \in K^{n}$ to the identity matrix yields the square matrix matrix $\left(I_{n}, v\right) \oplus e_{n+1} \in \mathcal{M}_{n+1}(K)$. As mentioned above, all square matrices are further embedded into $K[\mathcal{M}]$ via $\mathcal{M}_{n}(K) \hookrightarrow \mathcal{M}_{n+1}(K)$.

In the classical setting $K=\mathbb{R}$, the canonical action of monoid $\mathcal{M}(\mathbb{R})$ on the ascending $\mathbb{R}$-algebra $C^{\infty}\left(\mathbb{R}^{\infty}\right)$ is defined as follows. For given $M \in \mathcal{M}_{k}(\mathbb{R})$ and $f \in C^{\infty}\left(\mathbb{R}^{l}\right)$, we set $n=\max (k, l)$ so that we may take $M \in \mathcal{M}_{n}(\mathbb{R})$ and $f \in C^{\infty}\left(\mathbb{R}^{n}\right)$ via the corresponding embeddings. Then we think of $M$ as effecting the change of variables

$$
\begin{gathered}
\bar{x}_{1}=m_{11} x_{1}+\cdots+m_{1 n} x_{n}, \\
\vdots \\
\bar{x}_{n}=m_{n 1} x_{1}+\cdots+m_{n n} x_{n},
\end{gathered}
$$

and we define the action $\mathcal{M}_{n}(\mathbb{R}) \times C^{\infty}\left(\mathbb{R}^{n}\right) \rightarrow C^{\infty}\left(\mathbb{R}^{n}\right)$ by $(M, f) \mapsto f \circ M$. Via the embeddings, this yields the desired action $\mathcal{M}(\mathbb{R}) \times C^{\infty}\left(\mathbb{R}^{\infty}\right) \rightarrow C^{\infty}\left(\mathbb{R}^{\infty}\right)$.

Let us now turn to the general case of a contravariant monoid action $K[\mathcal{M}] \times \mathcal{F} \rightarrow \mathcal{F}$, meaning a monoid homomorphism $K[\mathcal{M}]^{*} \rightarrow \operatorname{AlgHom}_{K}(\mathcal{F})$, where $K[\mathcal{M}]^{*}$ denotes the opposite monoid of $K[\mathcal{M}]$. We require the following natural compatibility condition. For given $M \in \mathcal{M}(K)$, we write $M^{*}$ for the induced mapping $\mathcal{F} \rightarrow \mathcal{F}$. Moreover, we write $M_{\lrcorner n}$ for the $n$-th cut-off substitution, whose first $n$ rows coincide with those of $M$ while the subsequent ones are $e_{n+1}, e_{n+2}, \ldots$ In $C^{\infty}\left(\mathbb{R}^{\infty}\right)$ this means $M_{\lrcorner n}^{*}$ substitutes only in the first $n$ variables while leaving the remaining ones invariant. We call the action straight if $M^{*} f=M_{\lrcorner n}^{*} f$ for all $M \in \mathcal{M}(K)$ and $f \in \mathcal{F}_{n}$. The canonical action on $C^{\infty}\left(\mathbb{R}^{\infty}\right)$ is of course straight while for example shifting the filtration to $\mathcal{F}_{n}:=C^{\infty}\left(\mathbb{R}^{n+1}\right)$ leads to an action that is not straight.

Another crucial property of the classical example $C^{\infty}\left(\mathbb{R}^{\infty}\right)$ is that evaluation of a function $f \in C^{\infty}\left(\mathbb{R}^{n}\right)$ at $x_{n}=\xi \in \mathbb{R}$ leaves a function in $C^{\infty}\left(\mathbb{R}^{n-1}\right)$. In the general case we 
shall require this only for evalation at $\xi=0$, which can be described as the action $E_{n}^{*}$, where $E_{n}:=I-e_{n n} \in \mathcal{M}_{n}(K)$ is the $n$-th evaluation matrix (at zero). Adding this requirement to straightness, we arrive at the following axiomatization of multivariate functions.

Definition 2.1. An ascending $K$-algebra $\left(\mathcal{F}_{n}\right)$ is called a $K$-hierarchy if it has a straight contravariant monoid action of $\mathcal{M}(K)$ such that $M^{*}\left(\mathcal{F}_{n}\right) \subseteq \mathcal{F}_{n}$ for all $M \in \mathcal{M}_{n}(K)$ and $E_{n}^{*}\left(\mathcal{F}_{n}\right) \subseteq \mathcal{F}_{n-1}$. We write $\mathcal{F}$ for the direct limit of $\left(\mathcal{F}_{n}\right)$. By abuse of language, we refer also to $\mathcal{F}$ as a hierarchy.

In detail, a contravariant action means each $M^{*}: \mathcal{F} \rightarrow \mathcal{F}$ is a homomorphism of $K$ algebras that restricts to a homomorphism $M^{*}: \mathcal{F}_{n} \rightarrow \mathcal{F}_{n}$ whenever $M \in \mathcal{M}_{n}(K)$. Moreover, we have the usual action axioms $I^{*}=1_{\mathcal{F}}$ and $(M \tilde{M})^{*}=\tilde{M}^{*} M^{*}$. Note also that we have assumed $\mathcal{F}_{0}=K$, which implies that the action on $\mathcal{F}_{0}$ is trivial (since it fixes $1 \in K$ ).

In a $K$-hierarchy $\left(\mathcal{F}_{n}\right)$, we can define the following dependency lattice for expressing that some functions depend only on certain variables $x_{\alpha_{1}}, \ldots, x_{\alpha_{k}}$. For convenience, let us identify strictly increasing sequences $\alpha_{1}<\cdots<\alpha_{k}$ with finite subsets $\left\{\alpha_{1}, \ldots, \alpha_{k}\right\} \subset \mathbb{N}$; we will use the notation $\left(\alpha_{1}, \ldots, \alpha_{k}\right)$ for either of them. Furthermore, we shall identify permutations $\pi \in S_{n}$ with the permutation matrices $\left(\pi\left(e_{1}\right), \ldots, \pi\left(e_{n}\right)\right) \in \mathcal{M}_{n}(K)$. Let $S_{\infty}:=\bigcup_{n \in \mathbb{N}} S_{n}$ be the group of all permutations with finite support (those fixing all but finitely many elements of $\mathbb{N}$ ). Then we have an embedding $S_{\infty} \hookrightarrow \mathcal{M}(K)$. However, note that the action on column vectors is accordingly

$$
\pi\left(\begin{array}{c}
v_{1} \\
\vdots \\
v_{n}
\end{array}\right)=\left(\begin{array}{c}
v_{\tilde{\pi}(1)} \\
\vdots \\
v_{\tilde{\pi}(n)}
\end{array}\right)
$$

where $\tilde{\pi}$ is the inverse of $\pi$. We introduce the $K$-subalgebras

$$
\mathcal{F}_{\alpha}:=\left\{f \in \mathcal{F} \mid \pi^{*} f \in \mathcal{F}_{k}\right\}
$$

where $\pi:=\pi_{\alpha}: \mathbb{N} \rightarrow \mathbb{N}$ is the unique permutation with finite support sending $j$ to $\alpha_{j}$ for $j=1, \ldots, k$ while restricting to an increasing map $\mathbb{N} \backslash\{1, \ldots, k\} \rightarrow \mathbb{N} \backslash \alpha$. (In fact, the action of $\pi$ outside of $\{1, \ldots, k\}$ is immaterial because of the straightness assumption.) By convention we set $\mathcal{F}_{\emptyset}=\mathcal{F}_{0}$. One checks immediately, using the straightness of the action, that $\alpha \mapsto \mathcal{F}_{\alpha}$ is a monotonic map (in the sense that it preserves inclusions). Hence we may extend it to arbitrary $\alpha \subseteq \mathbb{N}$ by defining

$$
\mathcal{F}_{\alpha}=\bigcup_{n=1}^{\infty} \mathcal{F}_{\left(\alpha_{1}, \ldots, \alpha_{n}\right)} .
$$

This yields a complete bounded lattice $\left(\mathcal{F}_{\alpha}\right)$ with $\mathcal{F}_{\alpha} \sqcup \mathcal{F}_{\beta}=\mathcal{F}_{\alpha \cup \beta}$ and $\mathcal{F}_{\alpha} \sqcap \mathcal{F}_{\beta}=\mathcal{F}_{\alpha \cap \beta}$, with bottom element $\mathcal{F}_{\emptyset}=K$ and top element $\mathcal{F}_{\mathbb{N}}=\mathcal{F}$. Moreover, the lattice is complemented with $\mathcal{F}_{\alpha}^{\prime}=\mathcal{F}_{\mathbb{N} \backslash \alpha}$. Intuitively, $\mathcal{F}_{\alpha}$ captures those functions that depend at most on the variables specified in $\alpha$ and $\mathcal{F}_{\alpha}^{\prime}$ those that do not depend on these variables. 
The usual substitution notation $f\left(M_{11} x_{1}+\cdots+M_{1 n} x_{n}, \ldots, M_{n 1} x_{1}+\cdots+M_{n n} x_{n}\right)$ can be viewed as a convenient shorthand for $M^{*}(f)$, for a given substitution matrix

$$
M=\left(\begin{array}{ccc}
M_{11} & \cdots & M_{1 n} \\
\vdots & \ddots & \vdots \\
M_{n 1} & \cdots & M_{n n}
\end{array}\right)
$$

and $f \in \mathcal{F}_{n}$. While we will not employ this notation in the present paper (a more suitable notation is introduced in Section 4.1), it is certainly useful in a computational context.

The next step is to add Rota-Baxter operators and to describe their interaction with substitutions. This will lead to an algebraization of the well-known substitution rule for integrals. For basic definitions and properties of Rota-Baxter algebras we refer to [17, 18].

Let $R$ be a ring containing $\mathbb{Q}$, and let $(\mathcal{F}, P)$ be a Rota-Baxter algebra over $R$. Then we call $(\mathcal{F}, P)$ ordinary if $P$ is injective and $\operatorname{Im}(P) \dot{+} R=\mathcal{F}$ as $R$-modules. This is an algebraic way of describing $P$ as an integral operator on "univariate functions". In fact, we get an ordinary integro-differential algebra $(\mathcal{F}, d, P)$, where $d: \mathcal{F} \rightarrow \mathcal{F}$ is the unique derivation that sends $P(f)+c$ to $f$, for arbitrary $f \in \mathcal{F}$ and $c \in R$. Hence $1_{\mathcal{F}}-P \circ d$ is an algebra homomorphism $\mathcal{F} \rightarrow R$, which we call the associated evaluation of $(\mathcal{F}, d, P)$; it is the projector corresponding to the direct $\operatorname{sum} \operatorname{Im}(P) \dot{+} R=\mathcal{F}$.

Having an ordinary Rota-Baxter algebra has the added benefit of having the polynomial ring at our disposal. This holds for all ordinary integro-differential algebras [8, Prop. 3], but we give an independent proof here that does not make use of the derivation.

Lemma 2.2. Let $(\mathcal{F}, P)$ be an ordinary Rota-Baxter algebra over $R$. Then $x \mapsto P(1)$ defines an embedding $\left(R[x], \int_{0}^{x}\right) \hookrightarrow(\mathcal{F}, P)$ of Rota-Baxter algebras.

Proof. Since $\left(R[x], \int_{0}^{x}\right)$ is the initial object in the category of Rota-Baxter $R$-algebras [17, Cor. 3.2.4], there is a unique Rota-Baxter morphism $\iota: R[x] \rightarrow \mathcal{F}$, which clearly satisfies $\iota(x)=\iota\left(\int_{0}^{x} 1\right)=P(1)$. It remains to check that $\iota$ is injective, so we show that $\iota(p)=0$ implies $p=0$ for all polynomials $p \in R[x]$. We use induction on the degree of $p$. The induction base $p \in R$ is trivial since by definition $\iota$ acts as the identity on $R$. Now assume the claim for all polynomials of degree less than $k>0$, and take $p=p_{0}+p_{1} x+\cdots+p_{k} x^{k}$ with $\iota(p)=0$. Using the property $P(1)^{i}=i$ ! $P^{i}(1)$ from [17, Thm. 3.3.1], we obtain

$$
-p_{0}=\sum_{i=1}^{k} p_{i} \iota(x)^{i}=\sum_{i=1}^{k} i ! p_{i} P^{i}(1)=P\left(\sum_{i=0}^{k-1}(i+1) ! p_{i+1} P^{i}(1)\right) \in \operatorname{Im}(P),
$$

and $\operatorname{Im}(P) \dot{+} R=\mathcal{F}$ implies that $p_{0}$ as well as the expression on the left-hand side above vanish. Since $P$ is injective, this implies

$$
\iota\left(\sum_{i=0}^{k-1}(i+1) p_{i+1} x^{i}\right)=\sum_{i=0}^{k-1}(i+1) p_{i+1} P(1)^{i}=\sum_{i=0}^{k-1}(i+1) ! p_{i+1} P^{i}(1)=0,
$$

and the induction hypothesis yields $(i+1) p_{i}=0$ for all $i=0, \ldots, k-1$, and hence $p=0$. 
For an ascending algebra $\left(\mathcal{F}_{n}\right)$, it is natural to require an infinite collection of Rota-Baxter operators that we shall write as $\int^{x_{n}}$. Since we think of $\mathcal{F}_{1}$ as univariate functions, we shall require that $\int^{x_{1}}$ is an ordinary Rota-Baxter operator over $K=\mathcal{F}_{0}$. Analogous assumptions are imposed for $\int^{x_{n}}$ so that $\mathcal{F}_{n}$ is an ordinary Rota-Baxter algebra over $\mathcal{F}_{n-1}$. For the RotaBaxter operators $\int^{x_{n}}$ we shall now postulate the substitution rule for integration, which we shall only need for certain particular substitutions.

We introduce the following two special matrices. For $i<n$ we define the general transvection (= horizontal shear) in the $x_{i}$-direction as

$$
T_{i}(v)=\left(\begin{array}{ccccccc}
1 & & & & & & \\
& \ddots & & & & & \\
& & 1 & & & & \\
v_{1} & \cdots & v_{i-1} & 1 & v_{i+1} & \cdots & v_{n} \\
& & & & 1 & & \\
& & & & & \ddots & \\
& & & & & & 1
\end{array}\right) \leftarrow \text { row } i
$$

for a vector conveniently written as $v=\left(v_{1}, \ldots, v_{i-1}, v_{i+1}, \ldots, v_{n}\right)^{\top} \in K^{n-1}$. Similarly, we define the eliminant (= subdiagonal vertical shear)

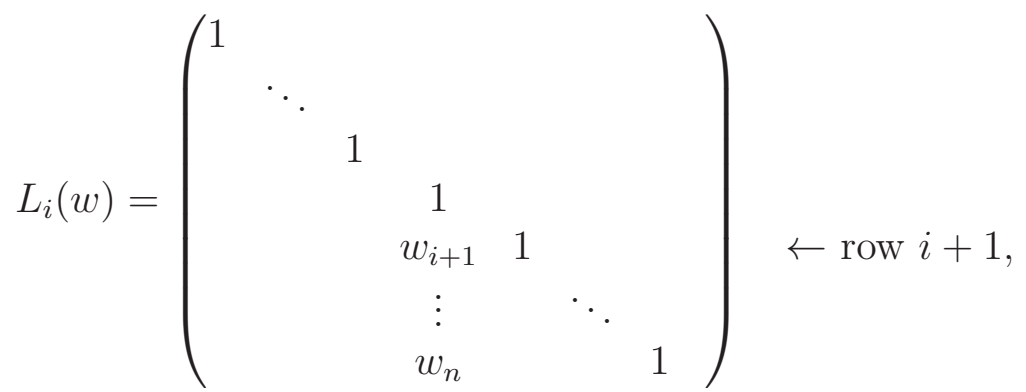

for a vector written as $w=\left(w_{i+1}, \ldots, w_{n}\right)^{\top} \in K^{n-i}$. When using the abbreviated variables $(x, y)=\left(x_{1}, x_{2}\right)$, we shall also write $T_{x}(v)$ and $T_{y}(v)$ for $T_{1}(v)$ and $T_{2}(v)$, respectively, and similar abbreviations will be in force for the eliminants. We note also the composition rule $L_{i}(w) L_{i}(\tilde{w})=L_{i}(w+\tilde{w})$ so that $L_{i}^{-1}(v)=L_{i}(-v)$ for eliminants in the same direction. 
However, for distinct directions we have the rules

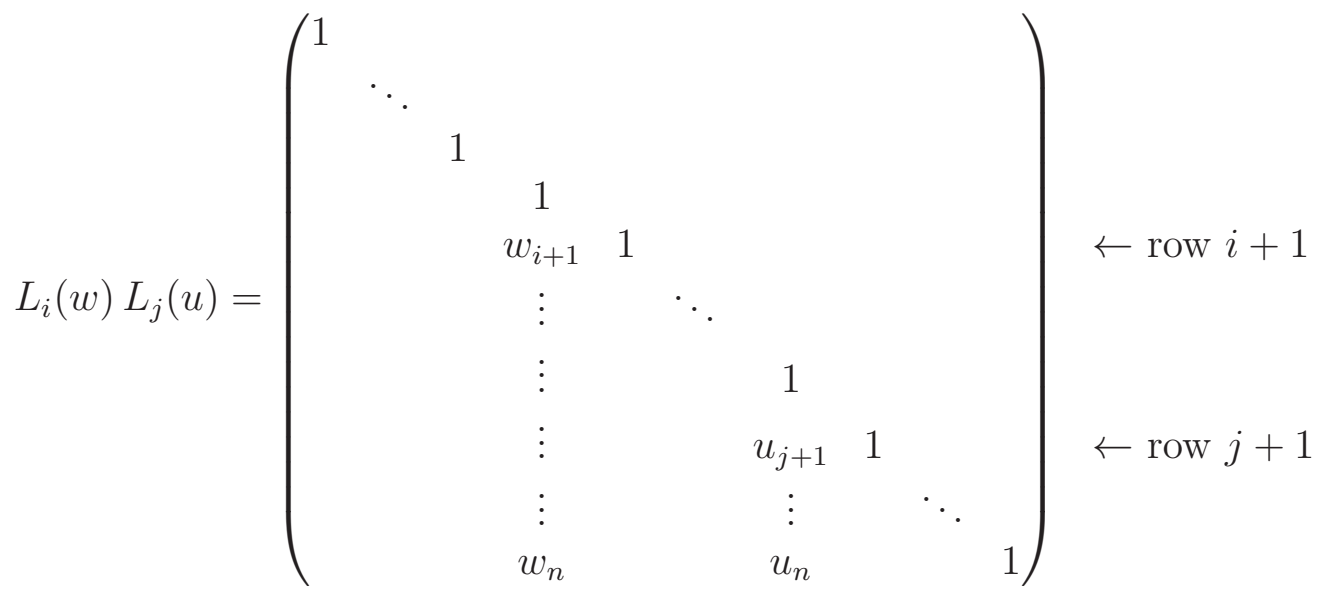

$$
L_{j}(u) L_{i}(w)=L_{i}\left(w^{\prime}\right) L_{j}(u)
$$

with $i<j$ and $w^{\prime}:=L_{j-i}(u) w \in K^{n-i}$. In particular, we note that the $L_{i}(w)$ and $L_{j}(u)$ do not commute.

We can now introduce the key structure that we shall use as an algebraic model of multivariate functions under integration and substitution. Roughly speaking, we shall need the subsitution rule for integration just for the following three matrix types: scalings, transvections, and eliminants.

Definition 2.3. A Rota-Baxter hierarchy $\left(\mathcal{F}_{n}, \int^{x_{n}}\right)_{n \in \mathbb{N}}$ consists of a $K$-hierarchy $\left(\mathcal{F}_{n}\right)$ and commuting Rota-Baxter operators $\left(\int^{x_{n}}\right)$ that satisfy the following axioms:

(a) We have $\int^{x_{n}} \mathcal{F}_{m} \subseteq \mathcal{F}_{m}$ and $\int^{x_{n}} \tilde{\mathcal{M}}_{m}(K)^{*}=\tilde{\mathcal{M}}_{m}(K)^{*} \int^{x_{n}}$ for $n \leq m$.

(b) Every $\left(\mathcal{F}_{n}, \int^{x_{n}}\right)$ is an ordinary Rota-Baxter algebra over $\mathcal{F}_{n-1}$ with evaluation $E_{n}^{*}$.

(c) We have $\tau^{*} \int^{x_{i}}=\int^{x_{j}} \tau^{*}$ for the transposition $\tau=(i j)$.

(d) For $\lambda \in K^{\times}$and $v=\left(0, v^{\prime}\right) \in K \oplus K^{n-2}$ we require the substitution rules

$$
\begin{aligned}
& \int^{x} \lambda^{*}=\lambda^{-1} \lambda^{*} \int^{x}, \\
& \int^{x} T_{x}\left(e_{1}\right)^{*}=\left(1-E_{x}^{*}\right) T_{x}\left(e_{1}\right)^{*} \int^{x}, \\
& \int^{x} L_{x}\left(e_{1}+v\right)^{*} \int^{x}=L_{y}^{-1}\left(v^{\prime}\right)^{*}\left[L_{x}\left(e_{1}\right)^{*}, \int^{x}\right] \int^{y} L_{y}\left(v^{\prime}\right)^{*} .
\end{aligned}
$$

We call (5), (6), (7) the diagonal, horizontal and vertical substitution rule, respectively.

Note that (5) describes the effect of scaling, (6) is essentially a one-dimensional substitution rule while (7) is the significant part of the two-dimensional substitution rule, which will be used for collapsing multiple integrals along the same direction. It turns out that all other instances of the substitution rule needed here can be inferred from the three instances (5)-(7). In particular, we note immediately that they imply the slightly more 
general cases of the three substitution rules

$$
\begin{aligned}
& \int^{x_{i}} d_{i, \lambda}=\lambda^{-1} d_{i, \lambda} \int^{x_{i}} \\
& \int^{x_{j}} T_{j}\left(e_{i}\right)^{*}=\left(1-E_{j}^{*}\right) T_{j}\left(e_{i}\right)^{*} \int^{x_{j}} \\
& \int^{x_{j}} L_{j}\left(e_{i}+v\right)^{*} \int^{x_{j}}=L_{i+1}^{-1}\left(v^{\prime}\right)^{*}\left[L_{j}\left(e_{i}\right)^{*}, \int^{x_{j}}\right] \int^{x_{i+1}} L_{i+1}\left(v^{\prime}\right)^{*}
\end{aligned}
$$

for any $i, j>0$, as one may readily check using conjugation by transpositions. In the vertical rule (10) we require $v=\left(0, v^{\prime}\right) \in K^{i-j+1} \oplus K^{n-i-1}$ and $j \leq i$.

2.2. Examples and Properties of Rota-Baxter Hierarchies. The vertical substitution rule (7) can be formulated in the following equivalent way, which is more symmetric and perhaps more natural (but less economical for our present purposes). This can be useful for proving that something is a Rota-Baxter hierarchy (as we will do in Theorem 3.7).

Lemma 2.4. Let $\Lambda \subset \mathbb{N}^{\times}$be an arbitrary finite index set with minimal element $\lambda \in \Lambda$ and complement $\Lambda^{\prime}=\Lambda \backslash\{\lambda\}$. Then

$$
\int^{x} L_{x}\left(\sum_{i \in \Lambda} e_{i}\right)^{*} \int^{x}=L_{\lambda+1}^{-1}\left(\sum_{i \in \Lambda^{\prime}} e_{i-\lambda}\right)^{*}\left[L_{x}\left(e_{\lambda}\right)^{*}, \int^{x}\right] \int^{x_{\lambda+1}} L_{\lambda+1}\left(\sum_{i \in \Lambda^{\prime}} e_{i-\lambda}\right)^{*}
$$

is equivalent to (7), assuming all other axioms of a Rota-Baxter hierarchy $\left(\mathcal{F}_{n}, \int^{x_{n}}\right)_{n \in \mathbb{N}}$.

Proof. It is easy to see that (11) is necessary. Indeed, let $n$ be the largest index of the set $\Lambda$. We may assume that $\lambda=1$ since otherwise we can set up a permutation $\pi \in S_{n}$ such that $\pi(\lambda)=1$; then conjugation of (11) by $\pi^{*}$ will ensure this. Now setting $v^{\prime}=\sum_{i \in \Lambda^{\prime}} e_{i}$ in (7) immediately yields formula (11). Note that the appearance of the $e_{i-\lambda}=e_{i-1}$ in the latter formula corresponds to the transition from $v \in K^{n-1}$ to $v^{\prime} \in K^{n-2}$ in the former.

For proving that (11) is sufficient, let $v=\left(0, v^{\prime}\right) \in K \oplus K^{n-2}$ be given, and let $\Lambda^{\prime} \subset \mathbb{N}^{\times}$ denote the set of all those $i$ with $v_{i}^{\prime} \neq 0$. Furthermore, set $\lambda=1$ and $\Lambda=\{1\} \cup \Lambda^{\prime}$. We use the scaling matrix $S=S_{3} \cdots S_{n} \in K^{n \times n}$ with

$$
S_{i+2}= \begin{cases}d_{i+2, v_{i}^{\prime}} & \text { if } v_{i} \neq 0 \\ I_{n} & \text { otherwise }\end{cases}
$$

for $1 \leq i \leq n-2$. Then we have $L_{x}\left(e_{1}+v\right)=S L_{x}\left(\sum_{i \in \Lambda} e_{i}\right) S^{-1}$ and hence

$$
L_{x}\left(e_{1}+v\right)^{*}=\left(S_{3}^{-1}\right)^{*} \cdots\left(S_{n}^{-1}\right)^{*} L_{x}\left(\sum_{i \in \Lambda} e_{i}\right)^{*} S_{3}^{*} \cdots S_{n}^{*} .
$$

Since the $S_{j}^{*},\left(S_{j}^{-1}\right)^{*} \in \tilde{\mathcal{M}}_{2}(K)^{*}$ commute with $\int^{x}$, substitution into (11) yields

$$
\begin{aligned}
\int^{x} L_{x}\left(e_{1}+v\right)^{*} \int^{x} & =\left(S^{-1}\right)^{*} L_{y}^{-1}\left(\sum_{i \in \Lambda^{\prime}} e_{i-1}\right)^{*}\left[L_{x}\left(e_{1}\right)^{*}, \int^{x}\right] \int^{y} L_{y}\left(\sum_{i \in \Lambda^{\prime}} e_{i-1}\right)^{*} S^{*} \\
& =\left(S^{-1}\right)^{*} L_{y}^{-1}\left(\sum_{i \in \Lambda^{\prime}} e_{i-1}\right)^{*} S^{*}\left[L_{x}\left(e_{1}\right)^{*}, \int\right] \int^{y}\left(S^{-1}\right)^{*} L_{y}\left(\sum_{i \in \Lambda^{\prime}} e_{i-1}\right)^{*} S^{*},
\end{aligned}
$$


where in the last step $S^{*}\left(S^{-1}\right)^{*}=1$ was inserted after $\int^{y}$, and $S^{*}$ was pushed left because the $S_{j}^{*}$ also commute with $\int^{y}$ and $L_{x}\left(e_{1}\right)^{*}$. Then we obtain $(7)$ since clearly $S L_{y}^{-1}\left(\sum\right) S^{-1}=$ $L_{y}^{-1}\left(v^{\prime}\right)$ as well as $S L_{y}\left(\sum\right) S^{-1}=L_{y}\left(v^{\prime}\right)$.

Let us now look at the most important example of a Rota-Baxter hierarchy - the algebra of smooth functions in several variables. This hierarchy contains also several important subhierarchies, in particular the analytic functions.

Example 2.5. In the classical setting we have $\mathcal{F}_{n}=C^{\infty}\left(\mathbb{R}^{n}\right)$ with Rota-Baxter operators

$$
\int^{x_{n}}: \mathcal{F} \rightarrow \mathcal{F}, \quad f \mapsto \int_{0}^{x_{n}} f\left(x_{1}, \ldots, x_{n-1}, \xi, x_{n+1}, \ldots\right) d \xi
$$

which clearly satisfy the mapping properties required in Item (a) above. Moreover, it is clear that every $\int^{x_{n}}$ is injective, and we have $\mathcal{F}_{n}=\mathcal{F}_{n-1} \dot{+} \operatorname{Im}\left(\int^{x_{n}}\right)$ since

$$
f\left(x_{1}, \ldots, x_{n}\right)=f\left(x_{1} \ldots, x_{n-1}, 0\right)+\int_{0}^{x_{n}} f^{\prime}\left(x_{1}, \ldots, x_{n-1}, \xi\right) d \xi
$$

and since $0=c\left(x_{1}, \ldots, x_{n-1}\right)+\int_{0}^{x_{n}} f\left(x_{1}, \ldots, x_{n-1}, \xi\right) d \xi$ implies $f=0$ upon differentiating with respect to $x_{n}$. Thus every $\left(\mathcal{F}_{n}, \int^{x_{n}}\right)$ is an ordinary Rota-Baxter algebra over $\mathcal{F}_{n-1}$ with evaluation $E_{n}^{*}: x_{n} \mapsto 0$, as required by Item (b). The transposition property of Item (c) is clear, while (8) follows by the substitution $\bar{\xi}=\lambda \xi$ in the integral $\int_{0}^{x_{1}} f\left(\lambda \xi, x_{2}, \ldots\right) d \xi$.

Now let us consider (6). Writing $T \equiv T_{x}\left(e_{1}\right)$, we have

$$
\begin{aligned}
& \int^{x_{1}} T^{*} f\left(x_{1}, x_{2}, x_{3}, \ldots\right)=\int_{0}^{x_{1}} f\left(\xi+x_{2}, x_{2}, x_{3}, \ldots\right) d \xi=\int_{x_{2}}^{x_{1}+x_{2}} f\left(\bar{\xi}, x_{2}, x_{3}, \ldots\right) d \bar{\xi} \\
& =\int_{0}^{x_{1}+x_{2}} f\left(\xi, x_{2}, x_{3}, \ldots\right) d \xi-\int_{0}^{x_{2}} f\left(\xi, x_{2}, x_{3}, \ldots\right) d \xi=\left(1-E_{x}^{*}\right) T^{*} \int^{x_{1}} f\left(x_{1}, x_{2}, x_{3}, \ldots\right)
\end{aligned}
$$

where the second equality employs the change of variables $\bar{\xi}=\xi+x_{2}$.

Finally, let us verify (7). Using the abbreviation $z_{k \ldots l} \equiv z_{k}, \ldots, z_{l}(k \leq l)$ for extracting and manipulating partial vectors with the obvious meaning, we have

$$
\begin{aligned}
& L_{2}\left(v^{\prime}\right)^{*} \int^{x_{1}} L_{x}\left(e_{1}+v\right)^{*} \int^{x_{1}} f\left(x_{1}, \ldots, x_{n}, \ldots\right) \\
& \quad=L_{2}\left(v^{\prime}\right)^{*} \int_{0}^{x_{1}} \int_{0}^{\eta} f\left(\xi, x_{2}+\eta, x_{3 \ldots n}+v_{3 \ldots n} \eta, \ldots\right) d \xi d \eta \\
& \quad=L_{2}\left(v^{\prime}\right)^{*} \int_{0}^{x_{1}} \int_{\xi+x_{2}}^{x_{1}+x_{2}} f\left(\xi, \bar{\eta}, x_{3 \ldots n}+v_{3 \ldots n}\left(\bar{\eta}-x_{2}\right), \ldots\right) d \bar{\eta} d \xi \\
& \quad=\int_{0}^{x_{1}} \int_{\xi+x_{2}}^{x_{1}+x_{2}} f\left(\xi, \eta, x_{3 \ldots n}+v_{3 \ldots n} \eta, \ldots\right) d \eta d \xi \\
& \quad=\int_{0}^{x_{1}} \int_{0}^{x_{1}+x_{2}} \ldots d \eta d \xi-\int_{0}^{x_{1}} \int_{0}^{\xi+x_{2}} \ldots d \eta d \xi
\end{aligned}
$$

where in the second equality the integration sweeps are swapped and the substitution $\bar{\eta}=$ $x_{2}+\eta$ is applied. It is easy to see that the first summand is $L_{x}\left(e_{1}\right)^{*} \int^{x_{1}} \int^{x_{2}} L_{y}\left(v^{\prime}\right)^{*} f$ and the second is $\int^{x_{1}} L_{x}\left(e_{1}\right)^{*} \int^{x_{2}} L_{y}\left(v^{\prime}\right)^{*} f$. 
This concludes the proof that $C^{\infty}\left(\mathbb{R}^{\infty}\right)$ is a Rota-Baxter hierarchy over $\mathbb{R}$. A similar proof will also work for the analytic subhierarchy $C^{\omega}\left(\mathbb{R}^{\infty}\right)$. This may be extended to complex variables as follows. Let $\int^{x}$ be the path integral from 0 to $x \in \mathbb{C}$. If $f \in C^{\omega}\left(\mathbb{C}^{\infty}\right)$ is a multivariate holomorphic function, we use the conjugates $\int^{x_{i}}=(1 i)^{*} \int^{x}(1 i)^{*}$ for creating the hierarchy of Rota-Baxter operators. One sees immediately that $C^{\omega}\left(\mathbb{C}^{\infty}\right)$ is a Rota-Baxter hierarchy over $\mathbb{C}$. (There is also an intermediate case where one considers only complex-valued $C^{\omega}$ functions on $\mathbb{R}^{n}$, but allowing complex substitutions. For example, $e^{i x}=\cos (x)+i \sin (x)$ may be interpreted in that way.)

Remark 2.6. The classical example provides a convenient geometrical interpretation of the three substitution rules (5), (6) and (7). The diagonal rule (5) describes the natural contravariant behavior when stretching or shrinking a coordinate axis.

The horizontal rule (6) says that integrating over the segment $[0, x] \times\{y\}$ after horizontally shearing it to $[y, x+y] \times\{y\}$ can be achieved by integrating over the whole sweep $[0, x+y] \times\{y\}$ minus the surplus $[0, y] \times\{y\}$. Its effect is that all axis-parallel line integrals may be started from the axis, generalizing the usual rule $\int_{a}^{b} \ldots d x=\int_{0}^{b} \ldots d x-$ $\int_{0}^{a} \ldots d x$ on the $x$-axis to all parallel segments.

The vertical rule (7) for $v=0$ may be seen to decompose an integral over the triangle $(0, y),(0, x+y),(x, x+y)$ as an integral over the rectangle $(0,0),(x, 0),(x, x+y),(0, x+y)$ minus an integral over the complement, namely the trapezoid $(0,0),(x, 0),(x, x+y),(0, y)$. Parametrizing the line segment from $(0, y)$ to $(x, x+y)$ as $\eta=s(\xi)$, the former integral is $\int_{0}^{x} \int_{0}^{y} \ldots d \eta d \xi$, the latter is $\int_{0}^{x} \int_{0}^{s(\xi)} \ldots d \eta d \xi$, so the effect is again to offset all integrals from the axes. The case $v \neq 0$ is similar except that the triangle is now tilted against the $x y$-plane, but the same decomposition as before appears in the orthogonal projection.

Finally, we should point out that the special case $\lambda=-1$ of $(5)$ means that $(-1)^{*}$ and $\int^{x}$ anti-commute. Thinking of Definition 2.3 as an axiomatization of the substitution rule of integration for the case of linear substitutions $A \in \mathcal{M}_{n}(\mathbb{R})$, the proper generalization to general "spaces" must be the signed integral over oriented manifolds rather than the socalled area formula of measure theory [14, §3.3][42, Thm. 2.6]. The latter would introduce absolute values whose algebraic treatment would be considerably more awkward.

Clearly, the notion of Rota-Baxter hierarchy gives rise to a category, with the morphisms respecting the linear action as well as the Rota-Baxter operators. In connection with this, several other notions can be built up in a natural fashion but in the present context we shall not need them. Let us only mention that a Rota-Baxter subhierarchy means that corresponding filtered components are Rota-Baxter subalgebras in the usual sense and that the linear action of $K[\mathcal{M}]$ coincides on them.

We proceed now by stating a few elementary consequences of the axioms. Though most of these are fairly obvious in the classical setting, we have to prove them here on the basis of our axioms for making sure that these include all the essential requirements for the theory to be developed.

Lemma 2.7. Every Rota-Baxter hierarchy $\left(\mathcal{F}_{n}, \int^{x_{n}}\right)$ satisfies the following properties. 
(a) For any $\alpha=\left(\alpha_{1}, \ldots, \alpha_{k}\right)$, there is an embedding

$$
\begin{aligned}
\iota_{\alpha}: K\left[X_{\alpha_{1}}, \ldots, X_{\alpha_{k}}\right] & \hookrightarrow \mathcal{F}_{\alpha} \\
X_{\alpha_{j}} & \mapsto x_{\alpha_{j}}:=\int^{x_{\alpha_{j}}} 1,
\end{aligned}
$$

and we have $\pi^{*} p\left(x_{\alpha_{1}}, \ldots, x_{\alpha_{k}}\right)=p\left(x_{\pi\left(\alpha_{1}\right)}, \ldots, x_{\pi\left(\alpha_{k}\right)}\right)$ for all permutations $\pi$ of $\left(\alpha_{1}, \ldots, \alpha_{k}\right)$.

(b) For $\pi \in S_{n}$ and $i \leq n$ we have $\pi^{*} \int^{x_{i}}=\int^{x_{j}} \pi^{*}$ with $j:=\pi(i)$. In particular, all $\int^{x_{i}}: \mathcal{F}_{(i)} \rightarrow \mathcal{F}_{(i)}$ are conjugates of $\int^{x_{1}}$ and hence ordinary Rota-Baxter operators.

(c) We have $\int^{x_{n}} c f=c \int^{x_{n}} f$ for all $c \in \mathcal{F}_{(n)}^{\prime}$ and $f \in \mathcal{F}$. In particular, $\int^{x_{n}} c=c x_{n}$.

(d) The embedding $\iota_{\alpha}$ of Item (a) is a homomorphism of Rota-Baxter algebras in the sense that $\iota_{\alpha} \circ \int_{0}^{X_{\alpha_{j}}}=\int^{x_{\alpha_{j}}} \circ \iota_{\alpha}$ for $j=1, \ldots, k$.

(e) If $M \in K[\mathcal{M}]$ vanishes in the $i$-th column, then $M^{*}(\mathcal{F}) \subseteq \mathcal{F}_{i}^{\prime}$.

(f) We have $E_{i}^{*} \int^{x_{i}}=0$ for all $i>0$.

Proof. (a) It suffices to consider $\alpha=(1, \ldots, n)$ since restricting to a subset of $\alpha$ induces another embedding, and permutations may be restricted accordingly (fixing the elements outside the subset).

By definition, $\iota_{n}:=\iota_{\alpha}$ is a homomorphism of $K$-algebras. We show that it is injective by induction on $n$, with the base case $n=1$ covered by Lemma 2.2. Since $\mathcal{F}_{\alpha}=\mathcal{F}_{n}$ is an ordinary Rota-Baxter algebra over $\mathcal{F}_{n-1}$, applying Lemma 2.2 again yields an embed$\operatorname{ding} \iota:\left(\mathcal{F}_{n-1}\left[X_{n}\right], \int_{0}^{X_{n}}\right) \hookrightarrow\left(\mathcal{F}_{n}, \int^{x_{n}}\right)$ defined by $X_{n} \mapsto x_{n}$. By the induction hypothesis we also have an embedding $\iota_{n-1}: K\left[X_{1}, \ldots, X_{n-1}\right] \hookrightarrow \mathcal{F}_{n-1}$ defined by $X_{i} \mapsto x_{i}(i<n)$. By the universal property of polynomials [5, Lem. 2.15], we obtain a unique $K$-linear map $\iota_{n}: K\left[X_{1}, \ldots, X_{n-1}\right]\left[X_{n}\right] \rightarrow \mathcal{F}_{n}$, which acts on coefficients via $\iota_{n-1}$ and sends $X_{n}$ to $x_{n}$. By [5, Lem. 2.16] and the injectivity of $\iota_{n-1}$, the map $\iota_{n}$ is injective iff

$$
\sum_{i=0}^{k} p_{i}\left(x_{1}, \ldots, x_{n-1}\right) x_{n}^{i}=0 \quad \text { implies } p_{0}, \ldots, p_{k}=0 \text {. }
$$

For showing (12) note that the left-hand side is the image of $\sum_{i} \iota_{n-1}\left(p_{i}\right) X_{n}^{i}$ under the embedding $\iota$, so that $p_{0}, \ldots, p_{k}=0$ follows from the injectivity of $\iota$ and $\iota_{n-1}$.

Now let $\pi \in S_{n}$ be an arbitrary permutation. Then Item (b) of this Lemma (whose proof below is independent) yields

$$
\pi^{*} p\left(x_{1}, \ldots, x_{n}\right)=p\left(\pi^{*} \int^{x_{1}} 1, \ldots, \pi^{*} \int^{x_{n}} 1\right)=p\left(\int^{x_{\pi(1)}} 1, \ldots, \int^{x_{\pi(n)}} 1\right)=p\left(x_{\pi(1)}, \ldots, x_{\pi(n)}\right)
$$

since clearly $\pi^{*} 1=1$.

(b) The general conjugate relation follows from Item (c) Definition 2.3 since every $\pi \in S_{n}$ is a product of transpositions. The last claim follows since $\int^{x_{i}}=\tau^{*} \int^{x_{1}} \tau^{*}$ with $\tau=(1 i)$ is clearly injective and for given $f \in \mathcal{F}_{(i)}$ we have $\tau^{*} f=f_{0}+\int^{x_{1}} f_{1}$ for some $f_{0} \in K$ and $f_{1} \in \mathcal{F}_{(1)}$, which implies $f=f_{0}+\int^{x_{i}} f_{i}$ for $f_{i}=\tau^{*} f_{1} \in \mathcal{F}_{(i)}$, and the decomposition is unique since the one for $\mathcal{F}_{(1)}=\mathcal{F}_{1}$ is.

(c) For a sufficiently large $k \geq n$ we have $f \in \mathcal{F}_{k}$ and $c \in \mathcal{F}_{(\alpha)}$ with $\alpha:=(1, \ldots, k) \backslash(n)$. So if $\pi$ is any permutation taking $\alpha_{i}$ to $i$, we have $\pi^{*} c \in \mathcal{F}_{k-1}$. We choose a permutation with $\pi(n)=k$ so that $\pi^{*} f \in \mathcal{F}_{k}$. Then Item (b) of Definition 2.3 yields $\int^{x_{k}}\left(\pi^{*} c \cdot \pi^{*} f\right)=$ 
$\left(\pi^{*} c\right) \int^{x_{k}} \pi^{*} f$. If $\tilde{\pi}$ is the inverse of $\pi$, left multipication by $\tilde{\pi}^{*}$ together with Item (b) of this Lemma gives the required identity.

(d) Again it suffices to consider $\alpha=(1, \ldots, n)$, so we show that $\iota_{n} \circ \int_{0}^{X_{i}}=\int^{x_{i}} \circ \iota_{n}$ for fixed $n$. In the case $i<n$ we use finite induction on $i$. The induction base $i=1$ is then covered by Lemma 2.2 and Item (c) above, so assume $i>1$. Then we have

$$
\begin{array}{r}
\iota_{n} \circ \int_{0}^{X_{i}}\left(\sum_{j=0}^{k} p_{j}\left(X_{1}, \ldots, X_{n-1}\right) X_{n}^{j}\right)=\iota_{n}\left(\sum_{j=0}^{k} X_{n}^{j} \int_{0}^{X_{i}} p_{j}\left(X_{1}, \ldots, X_{n-1}\right)\right) \\
\quad=\sum_{j=0}^{k} x_{n}^{j} \iota_{n-1}\left(\int_{0}^{X_{i}} p_{j}\left(X_{1}, \ldots, X_{n-1}\right)\right)=\sum_{j=0}^{k} x_{n}^{j} \int^{x_{i}} p_{j}\left(x_{1}, \ldots, x_{n-1}\right) \\
\quad=\int^{x_{i}} \sum_{j=0}^{k} p_{j}\left(x_{1}, \ldots, x_{n-1}\right) x_{n}^{j}=\int^{x_{i}} \circ \iota_{n}\left(\sum_{j=0}^{k} p_{j}\left(X_{1}, \ldots, X_{n-1}\right) X_{n}^{j}\right)
\end{array}
$$

where we have used the induction hypothesis in the third and Item (c) of this Lemma in the fourth equality. It remains to prove $\iota_{n} \circ \int_{0}^{X_{n}}=\int^{x_{n}} \circ \iota_{n}$ on $K\left[X_{1}, \ldots, X_{n}\right]=$ $K\left[X_{1}, \ldots, X_{n-1}\right]\left[X_{n}\right]$. To this end, recall that the embedding $\iota:\left(\mathcal{F}_{n-1}\left[X_{n}\right], \int_{0}^{X_{n}}\right) \hookrightarrow\left(\mathcal{F}_{n}, \int^{x_{n}}\right)$ from the above proof of Item (a) is a Rota-Baxter homomorphism by Lemma 2.2. Hence we obtain

$$
\begin{aligned}
\iota_{n} \circ \int_{0}^{X_{n}}\left(\sum_{j=0}^{k} p_{j}\left(X_{1}, \ldots, X_{n-1}\right) X_{n}^{j}\right)=\iota_{n}\left(\sum_{j=0}^{k} p_{j}\left(X_{1}, \ldots, X_{n-1}\right) X_{n}^{j+1} /(j+1)\right) \\
=\sum_{j=0}^{k} p_{j}\left(x_{1}, \ldots, x_{n-1}\right) x_{n}^{j+1} /(j+1)=\iota \circ \int_{0}^{X_{n}}\left(\sum_{j=0}^{k} p_{j}\left(x_{1}, \ldots, x_{n-1}\right) X_{n}^{j}\right) \\
=\int^{x_{n}} \circ \iota\left(\sum_{j=0}^{k} p_{j}\left(x_{1}, \ldots, x_{n-1}\right) X_{n}^{j}\right)=\int^{x_{n}} \circ \iota_{n}\left(\sum_{j=0}^{k} p_{j}\left(X_{1}, \ldots, X_{n-1}\right) X_{n}^{j}\right)
\end{aligned}
$$

where the Rota-Baxter property of $\iota$ has been employed in the fourth equality.

(e) We may assume $M \in \mathcal{M}_{n}(K)$ and $f \in \mathcal{F}_{n}$ for a sufficiently large $n \geq i$. Setting $\tau=$ $(i n) \in S_{n}$, we see that $M \tau$ has the last column zero so that $M \tau=(M \tau) E_{n}$. Hence we have also $\tau^{*} M^{*} f=E_{n}^{*}(M \tau)^{*} f \in \mathcal{F}_{n-1}$ by the evaluation property of hierarchies in Definition 2.1. Using the cyclic permutation

$$
\gamma=(i \mapsto i+1 \mapsto \cdots \mapsto n-1 \mapsto i) \in S_{n-1},
$$

we see that $\tau \gamma$ is a permutation that sends $k$ to $\alpha_{k}$ for $\alpha=(1, \ldots, i-1, i+1, \ldots, n)$, so by the definition of the dependency lattice we may infer $M^{*} f \in \mathcal{F}_{\alpha} \subseteq \mathcal{F}_{(i)}^{\prime}$ from $(\tau \gamma)^{*} M^{*} f=$ $\gamma^{*} \tau^{*} M^{*} f \in \mathcal{F}_{n-1}$. But the latter follows immediately from $\tau^{*} M^{*} f \in \mathcal{F}_{n-1}$ since $\gamma \in S_{n-1}$.

(f) Take $f \in \mathcal{F}_{n}$. If $n \leq i$ then $f \in \mathcal{F}_{i}$ and hence $E_{i}^{*} \int^{x_{i}} f=0$ follows from Item (b) of Definition 2.3. Otherwise let $\tau$ be the transposition $(n i)$ so that $E_{n}^{*} \int^{x_{n}} \tau^{*} f=0$ by the 
same token. Composing this on the left by $\tau^{*}$ gives $E_{i}^{*} \int^{x_{i}} f=0$ by Item (b) of this Lemma since $E_{i}=\tau E_{n} \tau$.

The horizontal substitution rule (9) can be generalized to the following result about arbitrary transvections.

Lemma 2.8. Let $\left(\mathcal{F}_{n}, \int^{x_{n}}\right)_{n \in \mathbb{N}}$ be a Rota-Baxter hierarchy over a field $K$. If $T=T_{i}(v)$ $i s$ any transvection along the $x_{i}$-axis with $\left(v_{1}, \ldots, v_{i-1}, v_{i+1}, \ldots, v_{n}\right) \in K_{n-1}$, then we have $\int^{x_{i}} T^{*}=\left(1-E_{i}^{*}\right) T^{*} \int^{x_{i}}$.

Proof. The general result follows from the case $i=1$ by conjugation with $(1 i) \in S_{n}$ and Item (c) of Definition 2.3. Hence we may assume $i=1$. If $T_{v}$ denotes the transvection (1) corresponding to $v=\left(v_{2}, \ldots, v_{n}\right) \in K_{n-1}$, we have the obvious relation $T_{v}=T_{v_{2} e_{2}} \cdots T_{v_{n} e_{n}}$ for the composition, so it suffices to consider $T_{a}:=T_{v}$ for $v=a e_{j}(j>1)$. We may furthermore assume that $a \neq 0$ since otherwise $T_{a}=I_{n}$, and the result follows by Item (f) in Lemma 2.7. If $S=d_{j, a}$ denotes the corresponding scaling in $x_{j}$-direction with inverse $\tilde{S}=$ $d_{j, 1 / a}$, we have $T_{a}=\tilde{S} T_{1} S$ and hence

$$
\begin{aligned}
\int^{x_{1}} T_{a}^{*} & =\int{ }^{x_{1}} S^{*} T_{1}^{*} \tilde{S}^{*}=S^{*} \int^{x_{1}} T_{1}^{*} \tilde{S}^{*}=S^{*}\left(1-E_{1}^{*}\right) T_{1}^{*} \int^{x_{1}} \tilde{S}^{*}=S^{*}\left(1-E_{1}^{*}\right) T_{1}^{*} \tilde{S}^{*} \int^{x_{1}}, \\
& =\left(1-E_{1}^{*}\right) T_{a}^{*} \int{ }^{x_{1}}
\end{aligned}
$$

where the second and fourth equality follows from Item (a) of Definition 2.3, the third from (9), and the last from $E_{1} S=S E_{1}$.

The vertical substitution rule can also be generalized in a way that will become important in the next secion - allowing a coefficient function within the integral. For handling this kind of situation we use the technique of slack variables. Before going through the formal proof, it may be helpful to recall this technique from Analysis. In fact, the verification of the vertical substitution rule in Example 2.5 can be generalized as follows:

$$
\begin{aligned}
& L_{2}\left(v^{\prime}\right)^{*} \int^{x_{1}} g\left(x_{1}\right) L_{x}\left(e_{1}+v\right)^{*} \int^{x_{1}} f\left(x_{1}, \ldots, x_{n}\right) \\
& \quad=L_{2}\left(v^{\prime}\right)^{*} \int_{0}^{x_{1}} g(\eta) \int_{0}^{\eta} f\left(\xi, x_{2}+\eta, x_{3 \ldots n}+v_{3 \ldots n} \eta\right) d \xi d \eta \\
& =L_{2}\left(v^{\prime}\right)^{*} \int_{0}^{x_{1}} \int_{\xi+x_{2}}^{x_{1}+x_{2}} g\left(\bar{\eta}-x_{2}\right) f\left(\xi, \bar{\eta}, x_{3 \ldots n}+v_{3 \ldots n}\left(\bar{\eta}-x_{2}\right)\right) d \bar{\eta} d \xi \\
& \quad=\int_{0}^{x_{1}} \int_{\xi+x_{2}}^{x_{1}+x_{2}} \bar{g}\left(x_{1}, \eta, x_{3}, \ldots, x_{n}, x_{2}\right) f\left(\xi, \eta, x_{3 \ldots n}+v_{3 \ldots n} \eta\right) d \eta d \xi \\
& =\int_{0}^{x_{1}} \int_{0}^{x_{1}+x_{2}} \ldots d \eta d \xi-\int_{0}^{x_{1}} \int_{0}^{\xi+x_{2}} \ldots d \eta d \xi
\end{aligned}
$$

The auxiliary function $\bar{g} \in \mathcal{F}_{n+1}$ is defined as $\bar{g}\left(x_{1}, x_{2}, \ldots, x_{n}, x_{n+1}\right):=g\left(x_{2}-x_{n+1}\right)$. Since the substitution induced by $J:=I_{n} \oplus e_{2}$ acts as $x_{n+1} \mapsto x_{2}$, it is now easy to check that the first summand is given by $J^{*} L_{x}\left(e_{1}\right)^{*} \int^{x_{1}} \int^{x_{2}} \bar{g} L_{y}\left(v^{\prime}\right)^{*} f$ and the second by $J^{*} \int^{x_{1}} L_{x}\left(e_{1}\right)^{*} \int^{x_{2}} \bar{g} L_{y}\left(v^{\prime}\right)^{*} f$. Thus we recover the same rule as before, apart from the presence of the multipliers $g, \bar{g}$ and the back substitution effected by $J^{*}$. The whole point 
is that the slack substitution $x_{2} \mapsto x_{n+1}$ allows us to temporarily "freeze" the variable $x_{2}$ so that it is not affected by integration. However, there is a price to pay for this: The identity is now restricted to $\mathcal{F}_{n}$ rather than being valid over all $\mathcal{F}$. This is reflected by the change from $f\left(x_{1}, \ldots, x_{n}, \ldots\right)$ in Example 2.5 to $f\left(x_{1}, \ldots, x_{n}\right)$ in the above verification. Practically speaking, this is not an essential restriction: The slack variable $x_{n+1}$ must be chosen large enough to prevent any conflict with the substitutions or integrals. Let us now prove the same result for general Rota-Baxter hierarchies.

Lemma 2.9. Let $\left(\mathcal{F}_{n}, \int^{x_{n}}\right)_{n \in \mathbb{N}}$ be a Rota-Baxter hierarchy over a field $K$, and let $g \in \mathcal{F}_{1}$ be any coefficient function. Then on $\mathcal{F}_{n}$ we have the identity

$$
\int^{x_{j}} g L_{j}\left(e_{i}+v\right)^{*} \int^{x_{j}}=L_{i+1}^{-1}\left(v^{\prime}\right)^{*}\left(I_{n} \oplus e_{i+1}\right)^{*}\left[L_{j}\left(e_{i}\right)^{*}, \int^{x_{j}}\right] \int^{x_{i+1}} \bar{g} L_{i+1}\left(v^{\prime}\right)^{*}
$$

for any $0<j \leq i$ and $v=\left(0, v^{\prime}\right) \in K^{i-j+1} \oplus K^{n-i-1}$. Here $\bar{g}:=\left(e_{i+1}-e_{n+1}\right)^{*} g \in \mathcal{F}_{n+1}$ is the transform of the coefficient function.

Proof. As before it will suffice to prove the case $i=j=1$ since the general case can then be recovered via conjugation by transpositions. Let $u \in \mathcal{F}_{n}$ be arbitrary but fixed. Since $g \in \mathcal{F}_{1}$, it is invariant under $L_{x}\left(e_{1}+v\right)^{*}$ by the straightness of the action. Moroever, setting $\tilde{g}:=\left(-e_{n+1}\right)^{*} g \in \mathcal{F}_{(n+1)}$ we have $g=E_{n+1}^{*} L_{x}\left(-e_{n}\right)^{*} \tilde{g}$, by straightness again since the first row of the matrix $\left(-e_{n+1} \oplus\left(0 I_{n}\right)\right) L_{x}\left(-e_{n}\right) E_{n+1} \in K^{(n+1) \times(n+1)}$ is $e_{1}$. Using this factorization we obtain

$$
\begin{aligned}
\int^{x} g L_{x}\left(e_{1}+v\right)^{*} \int^{x} u & =\int^{x} L_{x}\left(e_{1}+v\right)^{*} E_{n+1}^{*} L_{x}\left(-e_{n}\right)^{*} \tilde{g} \int^{x} u \\
& =\int^{x} E_{n+1}^{*} L_{x}\left(e_{1}+v-e_{n}\right)^{*} \tilde{g} \int^{x} u,
\end{aligned}
$$

where the first equality uses the multiplicativity of substitutions and the fact that $\int^{x} u \in \mathcal{F}_{n}$ is invariant under $E_{n+1}^{*}$ and $L_{x}\left(-e_{n}\right)^{*}$ by straightness once again; the second equality follows from $\left[L_{x}\left(e_{1}+v\right), E_{n+1}\right]=0$ and (3), where $L_{x}\left(e_{1}+v\right) \in K^{n \times n}$ is embedded into $K^{(n+1) \times(n+1)}$ via the filtration of $\mathcal{M}(K)$. Since $\tilde{g} \in \mathcal{F}_{(n+1)}$, we may move it in and out of the inner $\int^{x}$ by Item (c) of Lemma 2.7, while $E_{n+1} \in \tilde{\mathcal{M}}_{n}(K)$ and Item (a) of Definition 2.3 allows us to extract $E_{n+1}^{*}$ from the outer $\int^{x}$. Then we apply (7) to obtain

$$
\begin{aligned}
\int^{x} g L_{x}\left(e_{1}+v\right)^{*} \int^{x} u & =E_{n+1}^{*} \int^{x} L_{x}\left(e_{1}+v-e_{n}\right)^{*} \int^{x} \tilde{g} u \\
& =E_{n+1}^{*} L_{y}^{-1}\left(v^{\prime}-e_{n}\right)^{*}\left[L_{x}\left(e_{1}\right)^{*}, \int^{x}\right] \int^{y} L_{y}\left(v^{\prime}-e_{n}\right)^{*} \tilde{g} u,
\end{aligned}
$$

and we observe that $L_{y}^{-1}\left(v^{\prime}-e_{n}\right) E_{n+1}=\left(I_{n} \oplus e_{2}\right) L_{y}^{-1}\left(v^{\prime}\right)$ and $L_{y}\left(v^{\prime}-e_{n}\right)^{*} \tilde{g} u=\bar{g} L_{y}\left(v^{\prime}\right)^{*} u$, which may be verified by a short calculation (again using straightness for the latter).

The technique of slack variables gives considerable power to the notion of Rota-Baxter hierarchy. For example, choosing $\mathcal{F}=\bigcup_{n \geq 0} C^{\infty}\left(\mathbb{R}_{+}^{n}\right)$, the convolution of univariate functions $\star: \mathcal{F}_{1} \times \mathcal{F}_{1} \rightarrow \mathcal{F}_{1}$ can be defined by

$$
f \star g:=\left(I_{1} \oplus e_{x}\right)^{*} \int^{y}\left(e_{x}-e_{y}\right)^{*} f e_{y}^{*} g,
$$

which means $(f \star g)(x)=\int^{x} f(x-y) g(y) d y$, recovering the classical definition of the Duhamel convolution. Adding evaluations as in the ordinary case [35, §3], and limits as "evaluations at infinity" [1, §4], one can define and study integral transforms (e.g. Fourier, 
Laplace) in this algebraic framework. We will not pursue these topic in the present paper. For us, the main use of slack variables is to carry around coefficient functions within integral operators. This is a topic that we shall now investigate in some detail.

\section{Rota-Baxter Bialgebras}

Before we can build up the operator rings for Rota-Baxter hierarchies, we must first address the question of suitable coefficient domains. It turns out that such domains not only have an algebra structure (needed for composing multiplication operators) but also a coalgebra structure (needed for expressing basic linear substitutions) and a scaling action (in conjunction with the coalgebra this yields all linear substitutions).

3.1. Conceptualizing Linear Substitutions via Scaled Bialgebras. Again it is helpful to first look at the classical example $C^{\infty}\left(\mathbb{R}^{\infty}\right)$. Intuitively, we would like to simplify an integral operator like $\int^{y} f(x, y)$, acting as $u(x, y) \mapsto \int^{y} f(x, y) u(x, y)$, by pulling out of the integral those parts of $f(x, y)$ that depend only on $x$. For example, if $f(x, y)=(x+y)^{2}$ we would simplify

$$
\int^{y}(x+y)^{2} u(x, y)=x^{2} \int^{y} u(x, y)+2 x \int^{y} y u(x, y)+\int^{y} y^{2} u(x, y) .
$$

However, this kind of simplification is not possible for integrands like $f(x, y)=e^{x y}$ except if we are willing to use infinite expansion like $e^{x y}=\sum_{k}(1 / k !) x^{k} y^{k}$. In the terminology of integral equations, $f(x, y)=(x+y)^{2}$ is called a separated ${ }^{1}$ kernel, as opposed to the non-separated kernel $f(x, y)=e^{x y}$. Since we would like to refrain from using infinite sums (and hence topology), we will only allow separated kernels as coefficients in this paper. This will be made precise in Definition 3.9 below.

The expansion step (14) can be understood as the substitution $x \mapsto x+y$ on $g(x)=x^{2}$ to yield $f(x, y)=x^{2}+2 x y+y^{2}$. In other words, we have used the coproduct $\Delta: K[x] \rightarrow$ $K[x] \otimes K[x] \cong K[x, y]$ defined by $\Delta(x)=x \otimes 1+1 \otimes x$ so that $f=\Delta(g)$. Note that $\Delta$ is an algebra homomorphism; in fact, $K[x]$ has the structure of a bialgebra. For general properties of bialgebras we refer to $[9,12,41]$ and to $[17, \S 2]$. As we will make precise later (Definition 3.5), the coproduct interacts nicely with the Rota-Baxter structure.

Before we look at this interaction in more detail, it is apposite to focus first on the substitution structure of those bialgebras that provide "separated kernels" like the paradigmatic example $K[x, y]$ above. It turns out that we can build a $K$-hierarchy from a given bialgebra $\mathcal{H}$ from just one more ingredient, which we call scaling: an action of the ground field $K$ that will be extended to an action of the full matrix monoid $K[\mathcal{M}]$ on the tensor algebra over $\mathcal{H}$. In the classical example, this is the action $f(x) \mapsto f(\lambda x)$ for a function $f \in C^{\infty}(\mathbb{R})$ and a scalar $\lambda \in \mathbb{R}^{\times}$.

We formulate the basic properties of such an action in terms of the convolution product that we denote here by $\phi$. As stated in the Introduction, all bialgebras are assumed to be commutative and cocommutative. Recall [17, Thm. 2.3.4] that for a $K$-bialgebra $\mathcal{H}$ with product $\nabla$, unit 1 , coproduct $\Delta$ and counit I one defines the associative and commutative

\footnotetext{
${ }^{1}$ In Analysis, the term degenerated is most commonly used. From the viewpoint of Algebra, however, this term sounds too drastic so that we prefer the expression separated.
} 
operation $\phi$ on vector space endomorphism by setting $f \phi g=\nabla(f \otimes g) \Delta$. If $f$ and $g$ are bialgebra endomorphisms, then both $f \notin g$ and $f \circ g$ are. Writing $\operatorname{Bialg} \operatorname{Hom}_{K}(\mathcal{H})$ for the set of bialgebra $K$-endomorphisms, this yields two operations

$$
\text { ф, ०: } \operatorname{Bialg} \operatorname{Hom}_{K}(\mathcal{H}) \times \operatorname{BialgHom}_{K}(\mathcal{H}) \rightarrow \operatorname{BialgHom}_{K}(\mathcal{H}),
$$

which are clearly also $K$-linear. Moreover, one checks that $(f \phi g) \circ h=(f \circ h) \phi(g \circ h)$ and $h \circ(f \circ g)=(h \circ f) \phi(h \circ g)$. Hence $\left(\operatorname{BialgHom}_{K}(\mathcal{H}), \phi, \circ\right)$ is a commutative unital semiring. The neutral element with respect to $\phi$ is the composite $\mathrm{E}:=1 \circ \mathrm{J}$ of the unit $1: K \rightarrow \mathcal{H}$ and the counit $\mathrm{J}: \mathcal{H} \rightarrow K$. Of course, id $\mathrm{H}_{\mathcal{H}}: \mathcal{H} \rightarrow \mathcal{H}$ is the neutral element with respect to $\circ$.

Note that a (multiplicative) group action $K^{\times} \times \mathcal{H} \rightarrow \mathcal{H}$ is by definition a monoid homomorphism $\left(K^{\times}, \cdot, 1\right) \rightarrow\left(\operatorname{AlgHom}_{K}(\mathcal{H}), \circ, \mathrm{id}\right)$, which we shall write $\lambda \mapsto \lambda^{*}$. Clearly, we may extend it to a monoid homomorphism $K \rightarrow \operatorname{Alg} \operatorname{Hom}_{K}(\mathcal{H})$ by setting $0^{*}:=\mathrm{E}$. If the $\lambda^{*}$ are bialgebra homomorphisms, we refer to $K^{\times} \times \mathcal{H} \rightarrow \operatorname{BialgHom}_{K}(\mathcal{H})$ as a group biaction. For a scaling, we want this homomorphism to respect the convolution product.

Definition 3.1. Let $(\mathcal{H}, \nabla, 1, \Delta, \mathrm{J})$ be a $K$-bialgebra. Then a group biaction $K^{\times} \times \mathcal{H} \rightarrow \mathcal{H}$ is called a scaling if the map $(K,+, \cdot) \rightarrow\left(\operatorname{BialgHom}_{K}(\mathcal{H}), \phi, \circ\right)$, given by $\lambda \mapsto \lambda^{*}$ is a semiring homomorphism. In this case, we call $\mathcal{H}$ a scaled bialgebra.

In the sequel, we shall suppress the notation $\circ$ for the composition of endomorphisms. From the definition, we have the additive law $\left(\lambda_{1}+\lambda_{2}\right)^{*}=\lambda_{1}^{*} \phi \lambda_{2}^{*}=\nabla\left(\lambda_{1}^{*} \otimes \lambda_{2}^{*}\right) \Delta$. By induction, this generalizes immediately to

$$
\left(\lambda_{1}+\cdots+\lambda_{n}\right)^{*}=\nabla^{n}\left(\lambda_{1}^{*} \otimes \cdots \otimes \lambda_{n}^{*}\right) \Delta^{n}
$$

for all $n>0$, where $\nabla^{n}$ and $\Delta^{n}$ denote the obvious iterations (see below for the definition). Observe that the image $K^{*} \subseteq \operatorname{BialgHom}_{K}(\mathcal{H})$ of the scaling homomorphism $\lambda \mapsto \lambda^{*}$ is automatically a field. Hence we have a field homomorphism $K \rightarrow K^{*}$, and the group action $K^{\times} \times \mathcal{H} \rightarrow \mathcal{H}$ is faithful. Furthermore, note that $\mathcal{H}$ is a Hopf algebra with the antipode $(-1)^{*}: \mathcal{H} \rightarrow \mathcal{H}$ since this is clearly the convolution inverse of $\mathrm{id}_{\mathcal{H}}=1^{*}$.

We write $\mathfrak{H}(\mathcal{H})=\oplus_{i \geq 0} \mathcal{H}_{i}$ for the tensor algebra with grades $\mathcal{H}_{0}:=K$ and $\mathcal{H}_{i}=\mathcal{H}^{\otimes i}$ for $i>0$. The product on $\mathcal{H}$ is denoted by $\nabla: \mathcal{H} \otimes \mathcal{H} \rightarrow \mathcal{H}$, its iterations by $\nabla^{n}: \mathcal{H}_{n} \rightarrow \mathcal{H}$. Likewise, we write $\Delta^{n}: \mathcal{H} \rightarrow \mathcal{H}_{n}$ for the iterated coproduct defined by $\Delta^{1}=\mathrm{id}_{\mathcal{H}}$ and $\Delta^{n+1}=\left(\Delta^{n} \otimes \operatorname{id}_{\mathcal{H}}\right) \Delta$. Iterating coassociativity one obtains

$$
\left(\Delta^{m_{1}} \otimes \cdots \otimes \Delta^{m_{k}}\right) \Delta^{k}=\Delta^{m_{1}+\cdots+m_{k}}
$$

for all $m_{1}, \ldots, m_{k} \geq 0$ as in [28, Lem. 1.1.9(2)] with slightly different notation. Setting $m_{1}=2, m_{2}=\cdots=m_{n}=1$ yields the alternative recursion $\Delta^{n+1}=\left(\Delta \otimes \mathrm{id}^{n-1}\right) \Delta^{n}$ for computing the iterated coproduct. The counit axioms imply

$$
\left(\mathrm{E}^{\otimes(i-1)} \otimes \mathrm{id} \otimes \mathrm{E}^{\otimes(n-i)}\right) \Delta^{n}=1^{\otimes(i-1)} \otimes \mathrm{id} \otimes 1^{\otimes(n-i)},
$$

where the right-hand side is the embedding $\mathcal{H} \rightarrow \mathcal{H}_{n}$ defined by $f \mapsto 1^{\otimes(i-1)} \otimes f \otimes 1^{\otimes(n-i)}$. One checks also the iterated scaling commutations $\lambda^{*} \nabla^{n}=\nabla^{n}\left(\lambda^{*} \otimes \cdots \otimes \lambda^{*}\right)$ for the product and $\Delta^{n} \lambda^{*}=\left(\lambda^{*} \otimes \cdots \otimes \lambda^{*}\right) \Delta^{n}$ for the coproduct. 
The induced maps on the $n$-fold tensor products are then $\nabla^{n \otimes n}=\left(\nabla^{n}\right)^{\otimes n}: \mathcal{H}_{n}^{\otimes n} \rightarrow \mathcal{H}_{n}$ and $\Delta^{n \otimes n}=\left(\Delta^{n}\right)^{\otimes n}: \mathcal{H}_{n} \rightarrow \mathcal{H}_{n}^{\otimes n}$. Note that $\mathcal{H}_{n}$ is a bialgebra with (iterated) product $\nabla^{n \otimes n} \tau$ and (iterated) coproduct $\tau \Delta^{n \otimes n}$, where $\tau:=\tau_{n}: \mathcal{H}_{n}^{\otimes n} \rightarrow \mathcal{H}_{n}^{\otimes n}$ is the transposition

$$
\left(f_{11} \otimes \cdots \otimes f_{1 n}\right) \otimes \cdots \otimes\left(f_{n 1} \otimes \cdots \otimes f_{n n}\right) \mapsto\left(f_{11} \otimes \cdots \otimes f_{n 1}\right) \otimes \cdots \otimes\left(f_{1 n} \otimes \cdots \otimes f_{n n}\right) .
$$

If $a=\left(a_{1}, \ldots, a_{n}\right)$ is a column vector in $K^{n}$ or a row vector in $K_{n}$ we write $a^{\otimes}=a_{1}^{*} \otimes \cdots \otimes a_{n}^{*}$ for the corresponding scaling map on $\mathcal{H}_{n}$. Likewise, for a matrix $A \in \mathcal{M}_{n}(K)$ we write $A^{\otimes}=A_{1 \bullet}^{\otimes} \otimes \cdots \otimes A_{n \bullet}^{\otimes}$ for the scaling map on $\mathcal{H}_{n}^{\otimes n}$. If $\tilde{A}$ denotes the transpose matrix, we have $\tau A^{\otimes}=\tilde{A}^{\otimes} \tau$ or, in other words, $\tau\left(A_{1 \bullet}^{\otimes} \otimes \cdots \otimes A_{n \bullet}^{\otimes}\right)=\left(A_{\bullet 1}^{\otimes} \otimes \cdots \otimes A_{\bullet}^{\otimes}\right) \tau$.

We can now define the matrix action in terms of the coproduct and the scaling action. Note the appearance of a single $\tau$ in the expression for $M^{*}$ below: It allows us to interpret the left expression $\nabla^{n \otimes n} \tau$ as the product map on $\mathcal{H}_{n}$ or, via $\tau M^{\otimes}=\tilde{M} \tau$, the right expression $\tau \Delta^{n \otimes n}$ on $\mathcal{H}_{n}$ as the corresponding coproduct map.

Definition 3.2. Let $(\mathcal{H}, \nabla, 1, \Delta, \mathrm{J})$ be a scaled bialgebra. Then the induced matrix action $\mathcal{M}_{n}(K) \times \mathcal{H}_{n} \rightarrow \mathcal{H}_{n}$, written $(M, f) \mapsto M^{*} f$, is definded by $M^{*}=\nabla^{n \otimes n} \tau M^{\otimes} \Delta^{n \otimes n}$. The algebra $\mathfrak{H}(\mathcal{H})$ together with the corresponding action $K[\mathcal{M}] \times \mathfrak{H}(\mathcal{H}) \rightarrow \mathfrak{H}(\mathcal{H})$ is called the induced hierarchy for $\mathcal{H}$.

If we define the action $a^{*}: \mathcal{H} \rightarrow \mathcal{H}_{n}$ of a row $a=\left(a_{1}, \ldots, a_{n}\right) \in K_{n}$ by $a^{*}=a^{\otimes} \Delta^{n}$, the finite matrix action $\mathcal{M}_{n}(K) \times \mathcal{H}_{n} \rightarrow \mathcal{H}_{n}$ can be written as

$$
M^{*}\left(f_{1} \otimes \cdots \otimes f_{n}\right)=\left(M_{1 \bullet}^{*} f_{1}\right) \cdots\left(M_{n \bullet}^{*} f_{n}\right),
$$

where juxtaposition on the right-hand side denotes the iterated product in $\mathcal{H}_{n}$. The extension of the finite matrix action to $K[\mathcal{M}] \times \mathfrak{H}(\mathcal{H}) \rightarrow \mathfrak{H}(\mathcal{H})$ is to be understood as in Section 2: Every matrix of $K[\mathcal{M}]$ is of the block form $\left(\begin{array}{cc}A & 0 \\ 0 & I\end{array}\right)$ with $A \in \mathcal{M}_{r}(K)$ and $I$ the $\infty \times \infty$ identity matrix, while every $f \in \mathfrak{H}(\mathcal{H})$ is a finite sum of tensors of the form $f_{1} \otimes \cdots \otimes f_{s} \otimes 1 \otimes 1 \otimes \cdots$, so it suffices to choose $n$ as the maximum of those $r$ and $s$ and then use the action $\mathcal{M}_{n}(K) \times \mathcal{H}_{n} \rightarrow \mathcal{H}_{n}$. Let us now make sure that the induced hierarchy deserves its name.

Proposition 3.3. Let $(\mathcal{H}, \Delta, \mathrm{J})$ be a scaled bialgebra. Then the induced hierarchy $\mathfrak{H}(\mathcal{H})$ is a K-hierarchy in the sense of Definition 2.1.

Proof. The tensor algebra $\mathfrak{H}(\mathcal{H})$ is clearly an ascending algebra $\left(\mathcal{H}_{n}\right)$ with $\mathcal{H}_{n}=\mathcal{H}^{\otimes n}$ and direct limit $\mathfrak{H}(\mathcal{H})$. Moreover, it is clear from the definition of the induced matrix action that $M^{*}\left(\mathcal{H}_{n}\right) \subseteq \mathcal{H}_{n}$ for all $M \in \mathcal{M}_{n}(K)$.

Let us show that $E_{n}^{*}\left(\mathcal{H}_{n}\right) \subseteq \mathcal{H}_{n-1}$. We write $\iota_{j}:=\mathrm{E} \otimes \cdots \otimes \operatorname{id}_{\mathcal{H}} \otimes \cdots \otimes \mathrm{E}: \mathcal{H}_{n} \rightarrow \mathcal{H}_{n}$ with all entries $\mathrm{E}$ except for the $j$-th, which is $\mathrm{id}_{\mathcal{H}}$. Likewise, we write $\eta:=\mathrm{E} \otimes \cdots \otimes \mathrm{E}: \mathcal{H}_{n} \rightarrow \mathcal{H}_{n}$ for the tensor map having only E. Then $E_{n}^{\otimes}=\iota_{1} \otimes \cdots \otimes \iota_{n-1} \otimes \eta$, and (18) yields

$$
E_{n}^{*}\left(f_{1} \otimes \cdots \otimes f_{n-1} \otimes f_{n}\right)=\left(\iota_{1} \Delta^{n} f_{1}\right) \cdots\left(\iota_{n-1} \Delta^{n} f_{n-1}\right)\left(\eta \Delta^{n} f_{n}\right) .
$$

Since $\mathrm{E}: \mathcal{H} \rightarrow K \subseteq \mathcal{H}$, we have $\eta \Delta^{n} f_{n} \in K^{\otimes n} \cong K$, so it suffices to show $\iota_{j} \Delta^{n} f_{j} \in \mathcal{H}_{n-1}$ for all $j<n$. Writing $\tilde{\iota}_{j}: \mathcal{H}_{n-1} \rightarrow \mathcal{H}_{n-1}$ for the corresponding tensor maps with E everywhere except for the $j$-th entry $\operatorname{id}_{\mathcal{H}}$, we have $\iota_{j}=\tilde{\iota}_{j} \otimes \mathrm{E}$ and therefore

$$
\iota_{j} \Delta^{n}=\left(\tilde{\iota}_{j} \otimes \mathrm{E}\right)\left(\Delta^{n-1} \otimes \mathrm{id}_{\mathcal{H}}\right) \Delta=\left(\tilde{\iota}_{j} \Delta^{n-1} \otimes \operatorname{id}_{\mathcal{H}}\right)\left(\operatorname{id}_{\mathcal{H}} \otimes \mathrm{E}\right) \Delta=\tilde{\iota}_{j} \Delta^{n-1} \otimes 1,
$$


where the last identity uses the defining property of the counit in $\mathcal{H}$. But this clearly implies that $\iota_{j} \Delta^{n} f_{j}=\tilde{\iota}_{j} \Delta^{n-1} f_{j} \in \mathcal{H}_{n-1}$ for all $j<n$, as was required.

Next we prove that $\mathcal{M}_{n}(K) \times \mathcal{H}_{n} \rightarrow \mathcal{H}_{n}$ is a contravariant monoid action. For any matrices $M, \tilde{M} \in \mathcal{M}_{n}(K)$ and any function $f:=f_{1} \otimes \cdots \otimes f_{n} \in \mathcal{H}_{n}$ we must show

$$
(M \tilde{M})^{*}\left(f_{1} \otimes \cdots \otimes f_{n}\right)=\tilde{M}^{*} M^{*}\left(f_{1} \otimes \cdots \otimes f_{n}\right) .
$$

We start by computing $M^{*}\left(f_{1} \otimes \cdots \otimes f_{n}\right)$. Using sumless Sweedler notation, we have

$$
\Delta^{n \otimes n} f=\left(f_{1,(1)} \otimes \cdots \otimes f_{1,(n)}\right) \otimes \cdots \otimes\left(f_{n,(1)} \otimes \cdots \otimes f_{n,(n)}\right)=\bigotimes_{i} \bigotimes_{j} f_{i,(j)},
$$

where in the last expression (as in the rest of this proof) all indices range over $\{1, \ldots, n\}$. We have then $M^{\otimes} \Delta^{n \otimes n} f=\bigotimes_{i} \bigotimes_{j} M_{i j}^{*} f_{i,(j)}$ and further

$$
M^{*} f=\bigotimes_{j} \prod_{i} M_{i j}^{*} f_{i,(j)},
$$

with $\prod$ denoting the iterated product of $\mathcal{H}$. Using the fact that $\Delta^{n}$ is a bialgebra morphism, this implies $\Delta^{n \otimes n} M^{*} f=\bigotimes_{j} \prod_{i}\left(M_{i j}^{*}\right)^{\otimes n} \Delta^{n} f_{i,(j)}$. Now observe that the $\tilde{M}_{1 \bullet}^{\otimes}, \ldots, \tilde{M}_{n \bullet}^{\otimes}$ are algebra morphisms so that

$$
\begin{aligned}
\tilde{M}^{\otimes} \Delta^{n \otimes n} M^{*} f & =\bigotimes_{j} \prod_{i}\left(\bigotimes_{k}\left(M_{i j} \tilde{M}_{j k}\right)^{*}\right)\left(\Delta^{n} f_{i,(j)}\right)=\prod_{i} \bigotimes_{j}\left(\bigotimes_{k}\left(M_{i j} \tilde{M}_{j k}\right)^{*}\right)\left(\bigotimes_{k} f_{i,(j)(k)}\right), \\
& =\prod_{i}\left(\bigotimes_{j} \bigotimes_{k}\left(M_{i j} \tilde{M}_{j k}\right)^{*}\right)\left(\bigotimes_{j} \bigotimes_{k} f_{i,(j)(k)}\right)
\end{aligned}
$$

where the second step uses the definition of the product on $\mathcal{H}_{n}^{\otimes n}$. Applying the transposition $\tau$ to this equation will swap $j \leftrightarrow k$ in the double tensor products. However, note that $\bigotimes_{j} \bigotimes_{k} f_{i,(j)(k)}=\Delta^{n \otimes n} \Delta^{n} f_{i}=\Delta^{n^{2}} f_{i}$ by [28, Lem. 1.1.9(2)]. Since $\mathcal{H}$ is cocommutative, we have $\Delta^{N}=\pi \Delta^{N}$ for any $N>0$ and any permutation $\pi: \mathcal{H}_{N} \rightarrow \mathcal{H}_{N}$ of the tensor factors. In the special case of $\pi=\tau$ on $\mathcal{H}_{n^{2}} \cong \mathcal{H}_{n}^{\otimes n}$, this yields $\tau \Delta^{n \otimes n} \Delta^{n}=\Delta^{n \otimes n} \Delta^{n}$, so we can keep the argument $\Delta^{n \otimes n} \Delta^{n} f_{i}$. Since $\tau$ is furthermore a morphism of algebras, $\tilde{M}^{*} M^{*} f$ comes to

$$
\begin{gathered}
\prod_{i} \nabla^{n \otimes n}\left({\underset{k}{k}}_{j} \bigotimes_{j}\left(M_{i j} \tilde{M}_{j k}\right)^{*}\right)\left(\Delta^{n \otimes n} \Delta^{n} f_{i}\right)=\prod_{i}\left(\bigotimes_{k} \nabla^{n}\left(\bigotimes_{j}\left(M_{i j} \tilde{M}_{j k}\right)^{*}\right) \Delta^{n}\right) \Delta^{n} f_{i}, \\
=\prod_{i}\left(\bigotimes_{k}\left(\sum_{j} M_{i j} \tilde{M}_{j k}\right)^{*}\right) \Delta^{n} f_{i}=\prod_{i} \bigotimes_{k}(M \tilde{M})_{i k}^{*} f_{i,(k)},
\end{gathered}
$$

where we have used (15) in the last but one step. Comparing this with (19) and using again the definition of the product on $\mathcal{H}_{n}^{\otimes n}$, the claim follows.

Let us give some important examples of scaled bialgebras, which will also turn out to be admissible coefficient algebras for building a suitable ring of Rota-Baxter operators.

Example 3.4. The prototypical example of a scaled bialgebra is the polynomial ring $K[x]$. Its coproduct is given by $\Delta(x)=x \otimes 1+1 \otimes x$, which implies $\Delta\left(x^{n}\right)=\sum_{k=0}^{n}\left(\begin{array}{l}n \\ k\end{array}\right) x^{k} \otimes x^{n-k}$ 
on the canonical $K$-basis of $K[x]$. In other words, we have $\Delta p(x)=p(x+y)$ under the natural isomorphism $K[x] \otimes K[x] \cong K[x, y]$. This is a well-known example of a Hopf algebra, sometimes also called the binomial bialgebra [17, Ex. 2.2.3.2]. We use the scaling action $K^{\times} \times K[x] \rightarrow K[x]$ given by the substitutions $\lambda^{*} f(x)=f(\lambda x)$. It remains to check that the action $\lambda \mapsto \lambda^{*}$ respects the convolution product. Indeed, on the $K$-basis element $x^{m}$ we have

$$
\begin{aligned}
\left(\lambda^{*} \phi \mu^{*}\right)\left(x^{m}\right) & =\nabla\left(\lambda^{*} \otimes \mu^{*}\right) \Delta\left(x^{m}\right)=\sum_{k=0}^{m}\left(\begin{array}{c}
m \\
k
\end{array}\right) \nabla\left(\lambda^{*} \otimes \mu^{*}\right)\left(x^{k} \otimes x^{m-k}\right) \\
& =\sum_{k=0}^{m}\left(\begin{array}{c}
m \\
k
\end{array}\right) \nabla\left((\lambda x)^{k} \otimes(\mu x)^{m-k}\right)=\sum_{k=0}^{m}\left(\begin{array}{c}
m \\
k
\end{array}\right)(\lambda x)^{k}(\mu x)^{m-k}=(\lambda x+\mu x)^{m} \\
& =(\lambda+\mu)^{*} x^{m}
\end{aligned}
$$

as is required for a scaling. Under the aforementioned isomorphism $K[x]^{\otimes n} \cong K\left[x_{1}, \ldots, x_{n}\right]$, Definition 3.2 yields now the induced matrix action $\mathcal{M}_{n}(K) \times K\left[x_{1}, \ldots, x_{n}\right] \rightarrow K\left[x_{1}, \ldots, x_{n}\right]$ defined via (18) by $M^{*}\left(x_{1}^{k_{1}} \cdots x_{n}^{k_{n}}\right)=M_{1}^{*}\left(x^{k_{1}}\right) \cdots M_{n \bullet}^{*}\left(x^{k_{n}}\right)$ for the matrix $M \in \mathcal{M}_{n}(K)$ and exponent vector $\left(k_{1}, \ldots, k_{n}\right) \in \mathbb{N}^{n}$. Since $\Delta^{n} f(x)=f\left(x_{1}+\cdots+x_{n}\right)$, the row action

$$
\begin{aligned}
M_{i \bullet}^{*} x^{k_{i}} & =\left(M_{i 1}^{*} \otimes \cdots \otimes M_{i n}^{*}\right)\left(x_{1}+\cdots+x_{n}\right)^{k_{i}} \\
& =\sum_{l_{1}+\cdots+l_{n}=k_{i}}\left(\begin{array}{c}
k_{i} \\
l_{1}, \ldots, l_{n}
\end{array}\right)\left(M_{i 1}^{*} \otimes \cdots \otimes M_{i n}^{*}\right)\left(x_{1}^{l_{1}} \cdots x_{n}^{l_{n}}\right) \\
& =\sum_{l_{1}+\cdots+l_{n}=k_{i}}\left(\begin{array}{c}
k_{i} \\
l_{1}, \ldots, l_{n}
\end{array}\right)\left(M_{i 1} x_{1}\right)^{l_{1}} \cdots\left(M_{i n} x_{n}\right)^{l_{n}}=\left(M_{i 1} x_{1}+\cdots M_{i n} x_{n}\right)^{k_{i}},
\end{aligned}
$$

induces the overall action $M^{*}\left(x_{1}^{k_{1}} \cdots x_{n}^{k_{n}}\right)=\prod_{i}\left(\sum_{j} M_{i j} x_{j}\right)^{k_{i}}$ with the intended effect of a linear substitution $x_{i} \mapsto \sum_{j} M_{i j} x_{j}$. In other words, the column $\left(x_{1}, \ldots, x_{n}\right)$ gets multiplied on the left by the matrix $M \in \mathcal{M}_{n}(K)$.

Similar considerations apply to the larger ring of exponential polynomials $K\left[x, e^{K x}\right]$ and its variants (e.g. restricting the exponents to submonoids of $K$, like replacing $e^{K x}$ by $e^{\mathbb{N} x}$ ). Since the coproduct is an algebra morphism, it is sufficient to define it on the algebra generators $x$ and $e^{K x}$. Clearly, $K[x]$ should be a sub-bialgebra, so $\Delta$ coincides on $x$. For the exponential generators, one puts $\Delta e^{\alpha x}=e^{\alpha x} \otimes e^{\alpha x}$; so these are group-like elements unlike the primitive element $x$. Under the isomorphism $K\left[x, e^{K x}\right]^{\otimes n} \cong K\left[x_{1}, e^{K x_{1}}, \ldots, x_{n}, e^{K x_{n}}\right]$ we have again $\Delta^{n} f(x)=f\left(x_{1}, \ldots, x_{n}\right)$ for any $f \in K\left[x, e^{K x}\right]$. Moroever, it is easy to check that $(\lambda+\mu)^{*}=\lambda^{*} \phi \mu^{*}$, so we have a scaled bialgebra. As in the case of the polynomials, one sees that $M^{*}$ acts via the linear substitution $x_{i} \mapsto \sum_{j} M_{i j} x_{j}$.

3.2. Integration in Scaled Bialgebras. Let us now turn to the interaction between the substitution structure and Rota-Baxter operators. Here and henceforth we shall suppress the unit 1 and the product $\nabla$ of a bialgebra $(\mathcal{H}, \nabla, 1, \Delta, \mathrm{J})$. 
Definition 3.5. We call $\left(\mathcal{H}, \Delta, \mathrm{J}, \int\right)$ a Rota-Baxter bialgebra ${ }^{2}$ over $K$ if $\left(\mathcal{H}, \int\right)$ is an ordinary Rota-Baxter algebra over $K$ and $(\mathcal{H}, \Delta, \mathrm{J})$ is a bialgebra such that $\mathrm{E}:=1 \circ \mathrm{J}$ is the projector associated to $\operatorname{Im} \int \dot{+} K=\mathcal{H}$ and the horizontal substitution rule in the form

$$
\Delta \int=\left(\int \otimes \mathrm{id}\right) \Delta+(\mathrm{E} \otimes \mathrm{id}) \Delta \int
$$

is satisfied. We call $\left(\mathcal{H}, \Delta, \mathrm{J}, \int\right)$ a scaled Rota-Baxter bialgebra if it is further endowed with a scaling $K^{\times} \times \mathcal{H} \rightarrow \mathcal{F}, \lambda \mapsto \lambda^{*}$ subject to the diagonal substitution rule $\int \lambda^{*}=\lambda^{-1} \lambda^{*} \int$.

The condition that $\mathrm{E}$ be the projector associated to $\operatorname{Im} \int \dot{+} K=\mathcal{H}$ may also be expressed by $1 \circ \mathrm{J} \circ \int=0$ and $1 \circ \mathrm{J} \circ 1=1$, and this implies the direct sum. Hence we may also define a Rota-Baxter bialgebra as a a bialgebra with a Rota-Baxter operator that satisfies these two conditions along with (20).

As remarked above, a scaled bialgebra has the antipode $S:=(-1)^{*}$, which in the case of a scaled Rota-Baxter algebra satisfies $\int S+S \int=0$ by the diagonal substitution rule. This betrays the oriented nature of this notion of integration: In typical cases, like those described in Example 3.4, one has $S: f(x) \mapsto f(-x)$, so the integral picks up a sign under reflection (see also the remark at the end of Example 2.5).

Since the bialgebra $(\mathcal{H}, \Delta, \mathrm{J})$ is cocommutative by hypothesis, the horizontal substitution rule is also valid in its symmetric variant $\Delta \int=\left(\mathrm{id} \otimes \int\right) \Delta+(\mathrm{id} \otimes \mathrm{E}) \Delta \int$. Using (17) we can write both versions in the form

$$
\Delta \int f=\int^{x} \Delta f+\left(\int f\right)(y)=\int^{y} \Delta f+\left(\int f\right)(x)
$$

for functions $f \in \mathcal{H}_{1} \subset \mathfrak{H}(\mathcal{H})$. Here we have used the notation $h(x)=h \otimes 1$ and $h(y)=1 \otimes h$ for the two embeddings $\mathcal{H} \hookrightarrow \mathcal{H}_{2}$. Moreover, the horizontal substitution rule (20) can be iterated as follows.

Lemma 3.6. If $\left(\mathcal{H}, \Delta, \mathrm{J}, \int\right)$ is a Rota-Baxter bialgebra over $K$, we have

$$
\Delta^{n} \int=\left(\int \otimes \mathrm{id}^{\otimes(n-1)}\right) \Delta^{n}+\left(\mathrm{E} \otimes \mathrm{id}^{\otimes(n-1)}\right) \Delta^{n} \int
$$

for any $n>0$. Using the operations of $\mathfrak{H}(\mathcal{H})$, this may be written as $\Delta^{n} \int=\int^{x} \Delta^{n}+\mathrm{E}_{x} \Delta^{n}$.

Proof. Note that the case $n=1$ is trivial since $\Delta^{1}=$ id and $\mathrm{E} \int=0$. Hence we use induction over $n$ with the base case $n=2$ for $\Delta^{2}=\Delta$, given by the hypothesis (20). Hence assume (22) for a fixed $n \geq 2$; we show it for $n+1$. Using the definition of $\Delta^{n+1}$, the base case and then the induction hypothesis yields

$$
\begin{gathered}
\Delta^{n+1} \int=\left(\Delta^{n} \otimes \mathrm{id}\right)\left(\int \otimes \mathrm{id}\right) \Delta+\left(\Delta^{n} \otimes \mathrm{id}\right)(\mathrm{E} \otimes \mathrm{id}) \Delta \int=\left(\left(\left(\int \otimes \mathrm{id}^{\otimes(n-1)}\right) \Delta^{n}\right) \otimes \mathrm{id}\right) \Delta \\
+\left(\left(\left(\mathrm{E} \otimes \mathrm{id}^{\otimes(n-1)}\right) \Delta^{n} \int\right) \otimes \mathrm{id}\right) \Delta+\left(\Delta^{n} \otimes \mathrm{id}\right)(\mathrm{E} \otimes \mathrm{id}) \Delta \int .
\end{gathered}
$$

\footnotetext{
${ }^{2}$ Note that this notion is distinct from the Rota-Baxter coalgebra in sense of [24]. There may be interesting relations between the two concepts but this investigation will have to wait for future work. At this point, let us just mention that the standard integral on polynomials is a Rota-Baxter bialgebra (see Example 3.8 below) but not a Rota-Baxter coalgebra in the sense of [24]. Note also that the axiom (20) involves the counit, unlike the axiom in [24].
} 
Using (17) and the alternative recursion for $\Delta^{n}$ gives $\left(\mathrm{E} \otimes \mathrm{id}^{\otimes(n-1)}\right) \Delta^{n}=\left(1 \otimes \mathrm{id}^{\otimes(n-1)}\right) \Delta^{n-1}$ and $\left(\Delta^{n} \otimes \mathrm{id}\right)(\mathrm{E} \otimes \mathrm{id}) \Delta \int=1^{\otimes n} \otimes \int$. Combining this with the definition of $\Delta^{n+1}$ in the first summand we get

$$
\Delta^{n+1} \int=\left(\int \otimes \mathrm{id}^{\otimes n}\right) \Delta^{n+1}+\left(\left(\left(1 \otimes \mathrm{id}^{\otimes(n-1)}\right) \Delta^{n-1} \int\right) \otimes \mathrm{id}\right) \Delta+1^{\otimes n} \otimes \int .
$$

Using (20), its middle summand is

$$
\begin{aligned}
& \left(\left(\left(1 \otimes \mathrm{id}^{\otimes(n-1)}\right) \Delta^{n-1}\right) \otimes \mathrm{id}\right)\left(\int \otimes \mathrm{id}\right) \Delta=\left(\left(\left(1 \otimes \mathrm{id}^{\otimes(n-1)}\right) \Delta^{n-1}\right) \otimes \mathrm{id}\right)\left(\Delta \int-(\mathrm{E} \otimes \mathrm{id}) \Delta \int\right) \\
& \quad=1 \otimes \Delta^{n} \int-\left(\left(\left(1 \otimes \mathrm{id}^{\otimes(n-1)}\right) \Delta^{n-1} \mathrm{E}\right) \otimes \mathrm{id}\right) \Delta \int=1 \otimes \Delta^{n} \int-1^{\otimes n} \otimes \int
\end{aligned}
$$

where in the last step we have used $\Delta^{n-1} \mathbf{E}=\mathbf{E}^{\otimes(n-1)}$ and (17). Substituting this into $\Delta^{n+1} \int$, it remains only to check that $\left(\mathrm{E} \otimes \mathrm{id}^{\otimes n}\right) \Delta^{n+1} \int=1 \otimes \Delta^{n} \int$. Applying once again (17), and (16) with $m_{1}=2, m_{2}=n-1$, one sees immediately that $\left(\mathrm{E} \otimes \mathrm{id}^{\otimes n}\right) \Delta^{n+1}=1 \otimes \Delta^{n}$.

Theorem 3.7. Let $\left(\mathcal{H}, \Delta, \mathrm{J}, \int\right)$ be a scaled Rota-Baxter bialgebra. Then we obtain a RotaBaxer hierarchy $\left(\mathfrak{H}(\mathcal{H}), \int^{x_{n}}\right)_{n \in \mathbb{N}}$ with the operators $\int^{x_{n}}=\mathrm{id}^{\otimes(n-1)} \otimes \int$.

Proof. It is easy to see that the $\int^{x_{n}}$ are commuting Rota-Baxter operators on $\mathfrak{H}(\mathcal{H})$. Hence let us now check conditions (a) to (d) of Definition 2.3:

(a) Let $n \leq m$. From the definition of the Rota-Baxter operators, $\int^{x_{n}} \mathcal{H}^{\otimes m} \subseteq \mathcal{H}^{\otimes m}$ holds. For showing $\int^{x_{n}} \tilde{\mathcal{M}}_{m}(K)^{*}=\tilde{\mathcal{M}}_{m}(K)^{*} \int^{x_{n}}$, choose any

$$
\tilde{M}=\left(\begin{array}{cc}
I_{m} & 0 \\
0 & M
\end{array}\right) \quad \text { with } M \in \mathcal{M}_{r}(K)
$$

and $f \in \mathfrak{H}(\mathcal{H})$. We may choose $k \geq m+r$ such that $f \in \mathcal{H}^{\otimes k}$ with $f=f_{1} \otimes \cdots \otimes f_{k}$. By (18) we have

$$
\tilde{M}^{*} f=\left(e_{1}^{*} f_{1}\right) \cdots\left(e_{m}^{*} f_{m}\right)\left(\tilde{M}_{m+1, \bullet}^{*} f_{m+1}\right) \cdots\left(\tilde{M}_{m+r, \bullet}^{*} f_{m+r}\right)
$$


From (17) it follows that $\left(e_{1}^{*} f_{1}\right) \cdots\left(e_{m}^{*} f_{m}\right)=f_{1} \otimes \cdots \otimes f_{m} \in \mathcal{H}^{\otimes k}$. Unfolding the definition of $\tilde{M}_{m+j}^{*}(j=1, \ldots, r)$ and using coassociativity (16) we have

$$
\begin{aligned}
\tilde{M}_{m+j}^{*} f_{m+j} & =\left(\mathrm{E}^{\otimes m} \otimes M_{j \bullet}^{\otimes}\right) \Delta^{m+r} f_{m+j}=\left(\mathrm{id}^{\otimes m} \otimes M_{j \bullet}^{\otimes}\right)\left(\mathrm{E}^{\otimes m} \Delta^{m} \otimes \Delta^{r}\right) \Delta f_{m+j} \\
& =\mathrm{E}\left(f_{m+j,(1)}\right) 1^{\otimes m} \otimes M_{j \bullet}^{*} f_{m+j,(2)},
\end{aligned}
$$

where the last step uses the relation $\mathrm{E}^{\otimes m} \Delta^{m} g=\mathrm{E}(g) \in K \subseteq \mathcal{H}_{m}$ for $g \in \mathcal{H}$, which follows from (17). Altogether we obtain

$$
\tilde{M}^{*} f=\left(\prod_{j=1}^{r} \mathrm{E}\left(f_{m+j,(1)}\right)\right) f_{1} \otimes \cdots \otimes f_{m} \otimes \bigotimes_{j=1}^{r} M_{j \bullet}^{*} f_{m+j,(2)},
$$

from which it is clear that $\int^{x_{n}} \tilde{M}^{*} f=\tilde{M}^{*} \int^{x_{n}} f$ since the Rota-Baxter operator $\int^{x_{n}}$ affects only the prefix $f_{1} \otimes \cdots \otimes f_{m}$. 
(b) We must show that $\left(\mathcal{H}_{n}, \int^{x_{n}}\right)$ is an ordinary Rota-Baxter algebra over $\mathcal{H}_{n-1}$ with evaluation $E_{n}^{*}$. Computing the tensor kernel via [12, Prop. 2.17] we have

$$
\operatorname{Ker} \int^{x_{n}}=\left(\operatorname{Ker~id}^{\otimes(n-1)}\right) \otimes \mathcal{H}+\mathcal{H}_{n-1} \otimes \operatorname{Ker} \int=0 \otimes \mathcal{H}+\mathcal{H}_{n-1} \otimes 0
$$

since $\int$ is injective; we conclude that $\int^{x_{n}}$ is injective as well. Since $\left(\mathcal{H}, \int\right)$ is ordinary we have also $\mathcal{H}=K \dot{+} \operatorname{Im} \int$, which implies

$$
\mathcal{H}_{n}=\mathcal{H}_{n-1} \otimes \mathcal{H}=\left(\mathcal{H}_{n-1} \otimes K\right) \dot{+}\left(\mathcal{H}_{n-1} \otimes \operatorname{Im} \int\right) \cong \mathcal{H}_{n-1} \dot{+} \operatorname{Im} \int^{x_{n}}
$$

so $\left(\mathcal{H}_{n}, \int^{x_{n}}\right)$ is indeed ordinary over $\mathcal{H}_{n-1}$. The projector along the decomposition above is clearly id $^{\otimes(n-1)} \otimes \mathrm{E}$, which sends $f_{1} \otimes \cdots \otimes f_{n-1} \otimes f_{n}$ to $\mathrm{E}\left(f_{n}\right) f_{1} \otimes \cdots \otimes f_{n-1} \in \mathcal{H}_{n-1} \subseteq \mathcal{H}_{n}$. This agrees with

$$
E_{n}^{*}\left(f_{1} \otimes \cdots \otimes f_{n-1} \otimes f_{n}\right)=\left(e_{1}^{*} f_{1}\right) \cdots\left(e_{n-1}^{*} f_{n-1}\right)\left(0^{*} f_{n}\right)
$$

since $\left(e_{1}^{*} f_{1}\right) \cdots\left(e_{n-1}^{*} f_{n-1}\right)=f_{1} \otimes \cdots \otimes f_{n-1}$ again by $(17)$ and $0^{*} f_{n}=\mathrm{E}^{\otimes n} \Delta^{n} f_{n}=\mathrm{E}\left(f_{n}\right) \in K$ as in Item (a) of this proof.

(c) If $\tau=(i j)$ is any transposition, the property $\tau^{*} \int^{x_{i}}=\int^{x_{j}} \tau^{*}$ follows directly from the definition of the Rota-Baxter operators $\int^{x_{n}}$.

(d) Finally, we have to show the three instances of the substitution rule. Among these, the diagonal substitution rule (8) is an immediate consequence of the diagonal substitution rule in $\left(\mathcal{H}, \Delta, \mathrm{J}, \int\right)$ as specified in Definition 3.5.

For the horizontal substitution rule (6) note that $T_{x}\left(e_{1}\right)=\left(e_{1}+e_{2}\right) \oplus e_{2}$ so that

$$
T_{x}\left(e_{1}\right)^{*}(f \otimes g)=\left(e_{1}+e_{2}\right)^{*} f \cdot e_{2}^{*} g=(\Delta f)(1 \otimes g)=f_{(1)} \otimes f_{(2)} g,
$$

using again (17) for the second factor. Since $\int^{x}(f \otimes g)=\left(\int f\right) \otimes g$, using (20) yields

$$
\begin{aligned}
T_{x}\left(e_{1}\right)^{*} \int^{x}(f \otimes g)=\left(\Delta \int f\right)(1 \otimes g) & =\left(\int^{x} \Delta f+1 \otimes \int f\right)(1 \otimes g) \\
=\int f_{(1)} \otimes f_{(2)} g+1 \otimes\left(\int f\right) g & =\int^{x} T_{x}\left(e_{1}\right)^{*}(f \otimes g)+E_{x}^{*} T_{x}\left(e_{1}\right)^{*} \int^{x}(f \otimes g),
\end{aligned}
$$

where the last summand comes from observing that $E_{x}^{*} T_{x}\left(e_{1}\right)^{*}=\left(\begin{array}{ll}0 & 1 \\ 0 & 1\end{array}\right)^{*}$ and hence

$$
E_{x}^{*} T_{x}\left(e_{1}\right)^{*}\left(\int f\right) \otimes g=\left(e_{2}^{*} \int f\right)\left(e_{2}^{*} g\right)=(\mathrm{E} \otimes \mathrm{id}) \Delta \int f \cdot(\mathrm{E} \otimes \mathrm{id}) \Delta g=1 \otimes\left(\int f\right) g,
$$

where (17) was employed for the last step.

It remains to show the vertical substitution rule (7), which will need a bit more effort. By Lemma 2.4, we may assume that $v^{\prime} \in\{0,1\}^{n-2}$. Let us write $w=e_{1}+v \in\{0,1\}^{n-1}$, and let $\Lambda \subset \mathbb{N}^{\times}$the set containing 1 and all indices $i$ with $w_{i-1}=1$. By the definition of $\int^{x}$ and $\int^{y}$, it suffices to show $(7)$ on $\mathcal{H}_{n}$. Hence let $f=f_{1} \otimes \cdots \otimes f_{n} \in \mathcal{H}_{n}$ be arbitrary but fixed. We can write this as $\hat{f}^{0} \hat{f}^{1}$ with $\hat{f}^{j}=\hat{f}_{1}^{j} \otimes \cdots \otimes \hat{f}_{n}^{j}(j=0,1)$ and

$$
\hat{f}_{i}^{0}=\left\{\begin{array}{ll}
1 & \text { if } i \in \Lambda, \\
f_{i} & \text { otherwise, }
\end{array} \quad \text { and } \quad \hat{f}_{i}^{1}= \begin{cases}f_{i} & \text { if } i \in \Lambda, \\
1 & \text { otherwise. }\end{cases}\right.
$$

Observe that $\hat{f}^{0}=1 \otimes 1 \otimes \cdots$ commutes with $\int^{x}$ and $\int^{y}$. Using (17) we obtain also

$$
L_{x}(w)^{*} \int^{x} f=L_{x}(w)^{*}\left(\int f_{1}\right) \otimes f_{2} \otimes \cdots \otimes f_{n}=\hat{f}^{0} e_{1}^{*}\left(\int f_{1}\right) \prod_{i \in \Lambda \backslash\{1\}}\left(e_{1}+e_{i}\right)^{*} f_{i}
$$


and hence $\int^{x} L_{x}(w)^{*} \int^{x} f=\hat{f}^{0} \int^{x} L_{x}(w)^{*} \int^{x} \hat{f}^{1}$. A similar calculation shows that $\hat{f}^{0}$ commutes also with $L_{y}\left(v^{\prime}\right)^{*}, L_{y}^{-1}\left(v^{\prime}\right)^{*}$ and $L_{x}\left(e_{1}\right)^{*}$. Consequently we can pull out $\hat{f}^{0}$ both from the left and right-hand side of (7), and we may thus assume without loss of generality that $\hat{f}^{0}=1 \in \mathcal{H}_{n}$. Let $k$ be the cardinality of $\Lambda$. If $\tau \in S_{n}$ is any permutation sending $\Lambda$ to $\{1, \ldots, k\}$, conjugation by $\tau^{*}$ will reduce (7) to the case $f \in \mathcal{H}_{k} \subseteq \mathcal{H}_{n}$. Hence we may also assume that $k=n$ and $\Lambda=\{1, \ldots, n\}$. Then (23) shows that

$$
L_{x}(w)^{*} \int^{x} f=e_{1}^{*}\left(\int f_{1}\right) \prod_{i>1}\left(e_{1}+e_{i}\right)^{*} f_{i}
$$

Using again (17) we have $e_{1}^{*}\left(\int f_{1}\right)=\left(\int f_{1}\right) \otimes 1 \otimes \cdots \otimes 1 \in \mathcal{H}_{n}$. From the definition of the matrix action we have $\Delta=(2 i)^{*}\left(e_{1}+e_{i}\right)^{*}$, which implies

$$
\begin{gathered}
\int^{x} L_{x}(w)^{*} \int^{x} f=\int^{x}\left(\left(\int f_{1}\right) \prod_{i>1} f_{i,(1)} \otimes \bigotimes_{i>1} f_{i,(2)}\right)=\left(\int\left(\int f_{1}\right) \prod_{i>1} f_{i,(1)}\right) \otimes \underset{i>1}{\bigotimes} f_{i,(2)} \\
=\left(\left(\int f_{1}\right)\left(\int \prod_{i>1} f_{i,(1)}\right)\right) \otimes \bigotimes_{i>1} f_{i,(2)}-\int\left(f_{1} \int \prod_{i>1} f_{i,(1)}\right) \otimes \underset{i>1}{\bigotimes} f_{i,(2)} \\
=\left(\int f_{1}\right) \int^{x}\left(\prod_{i>1} f_{i,(1)} \otimes \bigotimes_{i>1} f_{i,(2)}\right)-\int^{x} f_{1}\left(\left(\int \prod_{i>1} f_{i,(1)}\right) \otimes \underset{i>1}{\bigotimes} f_{i,(2)}\right)
\end{gathered}
$$

where in the last but one step the Rota-Baxter axiom for $\mathcal{H}_{1}$ was applied, while the last step uses the embedding $\mathcal{H}_{1} \subset \mathcal{H}_{n}$. Turning now to the right-hand side of (7), we obtain

and hence by (20) also

$$
L_{y}\left(v^{\prime}\right)^{*} f=f_{1} \otimes f_{2} \prod_{i>2} f_{i,(1)} \otimes \bigotimes_{i>2} f_{i,(2)}
$$

$$
\begin{gathered}
L_{x}\left(e_{1}\right)^{*} \int^{x} \int^{y} L_{y}\left(v^{\prime}\right)^{*} f=L_{x}\left(e_{1}\right)^{*}\left(\left(\int f_{1}\right) \otimes\left(\int f_{2} \prod_{i>2} f_{i,(1)}\right) \otimes \bigotimes_{i>2} f_{i,(2)}\right) \\
=\left(\int f_{1}\right)\left(\Delta\left(\int f_{2} \prod_{i>2} f_{i,(1)}\right) \otimes \bigotimes_{i>2} f_{i,(2)}\right) \\
=\left(\int f_{1}\right) \int^{x}\left(f_{2,(1)} \prod_{i>2} f_{i,(1)(1)} \otimes f_{2,(2)} \prod_{i>2} f_{i,(1)(2)} \otimes \bigotimes_{i>2} f_{i,(2)}\right) \\
\left.\quad+\left(\int f_{1}\right) \otimes\left(\int f_{2} \prod_{i>2} f_{i,(1)}\right) \otimes \bigotimes_{i>2} f_{i,(2)}\right) .
\end{gathered}
$$

Using the antipode $S=(-1)^{*}$, we obtain further

$$
\begin{aligned}
& L_{y}^{-1}\left(v^{\prime}\right)^{*} L_{x}\left(e_{1}\right)^{*} \int^{x} \int^{y} L_{y}\left(v^{\prime}\right)^{*} f \\
& =\left(\int f_{1}\right) \int^{x}\left(f_{2,(1)} \prod_{i>2} f_{i,(1)(1)} \otimes f_{2,(2)} \prod_{i>2} f_{i,(1)(2)} S f_{i,(2)(1)} \otimes \bigotimes_{i>2} f_{i,(2)(2)}\right) \\
& \quad+\left(\int f_{1}\right) \otimes\left(\int f_{2} \prod_{i>2} f_{i,(1)}\right) \prod_{i>2} S f_{i,(2)(1)} \otimes \bigotimes_{i>2} f_{i,(2)(2)}
\end{aligned}
$$

Let us first study the left summand of (25), which we claim to be equal to the first term of (24). For this, it is sufficient to show that

$$
\prod_{i>2} f_{i,(1)(1)} \otimes \prod_{i>2} f_{i,(1)(2)} S f_{i,(2)(1)} \otimes \bigotimes_{i>2} f_{i,(2)(2)}=\prod_{i>2} f_{i,(1)} \otimes 1 \otimes \bigotimes_{i>2} f_{i,(2)} .
$$


From (16) we have $(\Delta \otimes \Delta) \Delta=\Delta^{4}=(\mathrm{id} \otimes \Delta \otimes \mathrm{id}) \Delta^{3}$, which may be used to rewrite the left-hand side as

$$
\begin{aligned}
& \prod_{i>2}(3 i)^{*}(\mathrm{id} \otimes \nabla \otimes \mathrm{id})(\Delta \otimes S \otimes \mathrm{id}) f_{i,(1)} \otimes f_{i,(2)(1)} \otimes f_{i,(2)(2)} \\
& =\prod_{i>2}(3 i)^{*}(\mathrm{id} \otimes \nabla \otimes \mathrm{id})(\mathrm{id} \otimes 2 \otimes S \otimes \mathrm{id})(\Delta \otimes \mathrm{id})(\mathrm{id} \otimes \Delta) \Delta f_{i}, \\
& =\prod_{i>2}(3 i)^{*}(\mathrm{id} \otimes \nabla(\mathrm{id} \otimes S) \otimes \mathrm{id})(\mathrm{id} \otimes \Delta \otimes \mathrm{id}) \Delta^{3} f_{i}=\prod_{i>2}(3 i)^{*}(\mathrm{id} \otimes \mathrm{E} \otimes \mathrm{id}) \Delta^{3} f_{i} \\
& =\prod_{i>2}(3 i)^{*}(\mathrm{id} \otimes \mathrm{E} \otimes \mathrm{id})(\Delta \otimes \mathrm{id}) \Delta f_{i}=\prod_{i>2}(3 i)^{*} f_{i,(1)} \otimes 1 \otimes f_{i,(2)}
\end{aligned}
$$

where the third step applies the identity $\nabla(\mathrm{id} \otimes S) \Delta=\mathrm{E}$, which is true because $(\mathcal{H}, \mathrm{J}, \Delta, S)$ is a Hopf algebra. The last expression follows from (17) and is clearly equal to the righthand side of (27).

We determine now the second term (26) of the commutator on the right-hand side of (7). To start with, we compute

$$
\begin{aligned}
& L_{x}\left(e_{1}\right)^{*} \int^{y} L_{y}\left(v^{\prime}\right)^{*} f=f_{1} \Delta\left(\int f_{2} \prod_{i>2} f_{i,(1)}\right) \otimes \bigotimes_{i>2} f_{i,(2)} \\
& =f_{1} \int^{x}\left(f_{2,(1)} \prod_{i>2} f_{i,(1)(1)} \otimes f_{2,(2)} \prod_{i>2} f_{i,(1)(2)} \otimes \bigotimes_{i>2}^{\bigotimes} f_{i,(2)}\right)+f_{1} \otimes\left(\int f_{2} \prod_{i>2} f_{i,(1)}\right) \otimes \bigotimes_{i>2}^{\otimes} f_{i,(2)},
\end{aligned}
$$

using once again (20); hence the required commutator term $L_{y}^{-1}\left(v^{\prime}\right)^{*} \int^{x} L_{x}\left(e_{1}\right)^{*} \int^{y} L_{y}\left(v^{\prime}\right)^{*} f$ is given by

$$
\begin{gathered}
\left(\int f_{1} \int f_{2,(1)} \prod_{i>2} f_{i,(1)(1)}\right) \otimes f_{2,(2)} \prod_{i>2} f_{i,(1)(2)} S f_{i,(2)(1)} \otimes \bigotimes_{i>2} f_{i,(2)(2)} \\
+\left(\int f_{1}\right) \otimes\left(\int f_{2} \prod_{i>2} f_{i,(1)}\right) \prod_{i>2} S f_{i,(2)(1)} \otimes \bigotimes_{i>2} f_{i,(2)(2)} .
\end{gathered}
$$

We notice that the second summand cancels with the second summand of the first commutator term (25). Therefore it remains to prove

$$
\begin{aligned}
& \left(\int f_{1} \int f_{2,(1)} \prod_{i>2} f_{i,(1)(1)}\right) \otimes f_{2,(2)} \prod_{i>2} f_{i,(1)(2)} S f_{i,(2)(1)} \otimes \bigotimes_{i>2} f_{i,(2)(2)} \\
& \quad=\int^{x} f_{1}\left(\int f_{2,(1)} \prod_{i>2} f_{i,(1)(1)} \otimes f_{2,(2)} \prod_{i>2} f_{i,(1)(2)} S f_{i,(2)(1)} \otimes \bigotimes_{i>2} f_{i,(2)(2)}\right) \\
& \quad=\int^{x} f_{1}\left(\left(\int \prod_{i>1} f_{i,(1)}\right) \otimes \bigotimes_{i>1} f_{i,(2)}\right),
\end{aligned}
$$

where the first equality uses only the definition of $\int^{x}$. But this clearly follows from

$$
f_{2,(1)} \prod_{i>2} f_{i,(1)(1)} \otimes f_{2,(2)} \prod_{i>2} f_{i,(1)(2)} S f_{i,(2)(1)} \otimes \bigotimes_{i>2} f_{i,(2)(2)}=\prod_{i>1} f_{i,(1)} \otimes \bigotimes_{i>1} f_{i,(2)},
$$

which is a trivial consequence of the identity (27) that we have shown above.

This concludes the proof that $\left(\mathfrak{H}(\mathcal{H}), \int^{x_{n}}\right)_{n \in \mathbb{N}}$ is a Rota-Baxter hierarchy. 
Example 3.8. Let us now make sure that the scaled bialgebras of our standard model (Example 3.4) are in fact scaled Rota-Baxter bialgebras under the natural choice of RotaBaxter operator. We do this for the exponential polynomials $K\left[x, e^{K x}\right]$, which includes the plain polynomials $K[x]$ as a Rota-Baxter subhierarchy.

The algebraic definition of the Rota-Baxter operator $\int$ on $K\left[x, e^{K x}\right]$ can be given either in recursive or in summation form; the latter is more amenable for our purposes. Hence we have for $k \in \mathbb{N}, \alpha \in K$ the formulae

$$
\begin{aligned}
\int x^{k} e^{\alpha x} & =\frac{(-1)^{k+1} k !}{\alpha^{k+1}}+\sum_{i=0}^{k} \frac{(-1)^{i} k \underline{\underline{i}}}{\alpha^{i+1}} x^{k-i} e^{\alpha x} \quad(\alpha \neq 0), \\
\int x^{k} & =\frac{x^{k+1}}{k+1}
\end{aligned}
$$

where $k^{i}=k ! /(k-i)$ ! denotes the falling factorial. For verifying that $\left(K\left[x, e^{K x}\right], \int\right)$ is a scaled Rota-Baxter bialgebra we have to check that it satisfies the diagonal substitution rule $\int \lambda^{*}=\lambda^{-1} \lambda^{*} \int(\lambda \neq 0)$ and the horizontal substitution rule (20). The former is immediate, so let us verify the latter in the form (21). For the $K$-basis vector $f=x^{k} e^{\alpha x}$ we compute

$$
\begin{aligned}
\Delta \int f & =\frac{(-1)^{k+1} k !}{\alpha^{k+1}}+\sum_{i=0}^{k} \sum_{j=0}^{k-i}\left(\begin{array}{c}
k-i \\
j
\end{array}\right) \frac{(-1)^{i} k^{\underline{i}}}{\lambda^{i+1}} x^{j} y^{k-i-j} e^{\alpha(x+y)} \\
\int^{x} \Delta f & =\sum_{l=0}^{k}\left(\begin{array}{c}
k \\
l
\end{array}\right) \frac{(-1)^{l+1} l !}{\alpha^{l+1}} y^{k-l} e^{\alpha y}+\sum_{l=0}^{k} \sum_{i=0}^{l}\left(\begin{array}{c}
k \\
l
\end{array}\right) \frac{(-1)^{i} l^{\underline{i}}}{\alpha^{i+1}} x^{l-i} y^{k-l} e^{\alpha(x+y)}, \\
\left(\int f\right)(y) & =\frac{(-1)^{k+1} k !}{\alpha^{k+1}}-\sum_{l=0}^{k} \frac{(-1)^{l+1} k^{l}}{\alpha^{l+1}} y^{k-l} e^{\alpha y}
\end{aligned}
$$

We observe that the constant terms of $\Delta \int f$ cancels with the one of $\left(\int f\right)(y)$, likewise the first term of $\int^{x} \Delta f$ with the second term of $\left(\int f\right)(y)$; thus it remains to show the two double sums equal. But this follows immediately by transforming the index $i$ of the outer sum in $\Delta \int f$ to $l=i+j$ and then swapping the summations. The verification for the $K$-basis vector $f=x^{k}$ is similar but simpler.

Since every ordinary Rota-Baxter algebra $\left(\mathcal{G}, \int\right)$ contains $K[x]$, its induced hierarchy contains the ascending algebra of polynomial rings $K\left[x_{1}, \ldots, x_{n}\right]$. These are closed under all integrators and linear substitutions, so one can always choose $K\left[x_{1}, x_{2}, \ldots\right]$ as the simplest coefficient domain. Another natural choice for the classical example $C^{\infty}\left(\mathbb{R}^{\infty}\right)$ is given by the exponential polynomials discussed in Example 3.8 above. Both of these are instances of admissible coefficient domains in the following sense.

Definition 3.9. A Rota-Baxter hierarchy is called separated if it is of the form $\mathfrak{H}(\mathcal{H})$ for some scaled Rota-Baxter bialgebra $\mathcal{H}$. If $\mathcal{F}$ is a fixed Rota-Baxter hierarchy, an admissible coefficient domain for $\mathcal{F}$ is a separated Rota-Baxter subhierarchy $\mathcal{G} \leq \mathcal{F}$. 
As remarked above, the minimal choice is to take $\mathcal{G}=\mathfrak{H}(K[x])$, which is an admissible coefficient algebra for any Rota-Baxter hierarchy $\left(\mathcal{F}, \int^{x_{n}}\right)_{n \in \mathbb{N}}$. We may view it as an analog of the prime field in any given field.

\section{The Ring of Partial Integral Operators}

We proceed now to the task of setting up a ring of partial integral operators and substitutions acting on a given Rota-Baxter hierarchy, and taking coefficients in any admissible coefficient domain. We will do this in two steps: first identifying first an ideal of suitable operator relations, then constructing the operator ring as a quotient algebra of the free algebra modulo the relation ideal.

Note the parallel development for rings of differential operators, where the $n$-th Weyl algebra $A_{n}(K)$ is built as a quotient of $K\left[x_{1}, \ldots, x_{n} ; \partial_{1}, \ldots, \partial_{n}\right]$ modulo $\left[\partial_{i}, x_{j}\right]=\delta_{i j}$. In the case of a single Rota-Baxter operator (without linear substitutions), the corresponding operator ring has been studied [19] in connection with representations of Rota-Baxter algebras.

4.1. Crucial Operator Relations. Logically speaking, all the operator relations that we shall now compile are in fact consequences of the axioms of Rota-Baxter hierarchies (and admissible coefficient algebras). But we need the operator relations in this special form since it facilitates the algorithmic treatment of partial integral operators and linear substitutions. Ultimately, we hope to have a noncommutative Gröbner basis for the relation ideal (see the end of Section 4.2).

First we need to clarify some notational conventions. Let $\left(\mathcal{F}_{n}, \int^{x_{n}}\right)_{n \in \mathbb{N}}$ be a fixed RotaBaxter hierarchy over $K$. For avoiding confusion with multiplication operators, we will from now on apply the alternative notation $f[M]$ for the action $M^{*}(f)$. If $M=(i j)$ is the matrix of a transposition, we write $f[i j]$ for $f[M]$, and parentheses are also dropped for embedded row vectors $M$. We may identify all elements $f \in \mathcal{F}$ with their induced multiplication operators $\mathcal{F} \rightarrow \mathcal{F}, u \mapsto f u$. Thus the operator $M^{*} f$ denotes the composition $u \mapsto M^{*}(f u)$ rather than the action $f[M]$. The basic operator relation $M^{*} f=f[M] M^{*}$ describes how substitutions interact with multiplication operators. Note that $f[M][\tilde{M}]=f[M \tilde{M}]$.

Let $\mathcal{G}$ be an admissible coefficient domain for $\mathcal{F}$. Then we have $\mathcal{G}=\mathfrak{H}(\mathcal{H}) \leq \mathcal{F}$ for a scaled Rota-Baxter bialgebra $\mathcal{H}$. Since the latter is determined by the coefficient domain as the first tensor grade $\mathcal{H}=\mathcal{G}_{1}$, we may omit reference to $\mathcal{H}$ altogether. If $f \in \mathcal{G}_{1}$ we shall write $f\left(x_{i}\right) \in \mathcal{G}_{(1)}$ as an intuitive shorthand for $(1 i)^{*} f$.

The crucial property of an admissible coefficient domain is that it provides tensor expansion all multivariate functions $g \in \mathcal{F}$ as per

$$
g=\sum_{\mu=1}^{r} g_{1, \mu} \otimes \cdots \otimes g_{n, \mu}
$$

for suitable $g_{1, \mu}, \ldots, g_{n, \mu} \in \mathcal{F}_{1}$, where we shall consistently use Greek indices for the components. For definiteness, we fix an ordered $K$-basis $\left(b_{i}\right)$ for $\mathcal{F}_{1}$, and we use the graded lexicographic ordering for the induced $K$-basis on $\mathcal{F}_{n}$. Then each summand above has the 
form $\lambda_{\mu} b_{i_{1}(\mu)} \otimes \cdots \otimes b_{i_{n}(\mu)}$ so that we may choose $g_{1, \mu}=\lambda_{\mu} b_{i_{1}(\mu)}$ and $g_{j, \mu}=b_{i_{j}(\mu)}$ for $j>1$. Consequently, each $g \in \mathcal{F}_{n}$ is uniquely described by the $n$ component sequences

$$
g\{j\}:=(1 j)^{*}\left(g_{j, 1}, \ldots, g_{j, r}\right) \in \mathcal{G}_{(j)}^{r} \quad(j=1, \ldots, n),
$$

where the number $r \in \mathbb{N}$ depends of course on the element $g \in \mathcal{F}_{n}$; its minimial value is the tensor rank of $g$. Note that we recover (28) by setting $g_{j, \mu}=(g\{j\})_{\mu}$ and replacing the tensor product by the product of $\mathcal{G}$. For $\alpha \subseteq\{1, \ldots, n\}$ we will also use the extended sequence notation

$$
g\{\alpha\}:=\prod_{j \in \alpha} g\{j\} \in \mathcal{G}_{\alpha}^{r}
$$

where the product on the right-hand side is taken componentwise. If $\alpha=\left(i_{1}, \ldots, i_{r}\right)$ we denote this briefly by $g\left\{i_{1}, \ldots, i_{r}\right\}$. For complements we write $g\{\alpha\}^{\prime}:=g\left\{\alpha^{\prime}\right\}$, where $\alpha^{\prime}$ is to be understood as $\{1, \ldots, n\} \backslash \alpha$.

We will employ component sequences in conjunction with the following variant of the Einstein summation convention: Unless stated otherwise, summation is implied over all Greek indices on a component sequence (over the index range of the component sequence). ${ }^{3}$ For example, the expansion (28) can be written as $g=g\{1\}_{\mu} g\{1\}_{\mu}^{\prime}$. Similarly, we have equivalent expansions like $g=g\{1\}_{\mu} g\{1,2\}_{\mu}^{\prime} g\{2\}_{\mu}$. The practical value of this convention will become apparent when component sequences are used in noncommutative operator expressions, as in the following result generalizing Lemma 2.7. Here and henceforth we employ the following notation for sequence substitution: If $\gamma \in \mathcal{F}_{\alpha}^{r}$ is an extended operator sequence and $M \in \mathcal{M}_{n}(K)$ any substitution, we write $\gamma[M]$ for the sequence with components $\gamma_{1}[M], \ldots, \gamma_{r}[M]$. In the sequel, indices $i, j$ range over $\{1, \ldots, n\}$.

Lemma 4.1. Let $\left(\mathcal{F}_{n}, \int^{x_{n}}\right)_{n \in \mathbb{N}}$ be a Rota-Baxter hierarchy over a field $K$, and $\mathcal{G}$ an admissible coefficient domain for $\mathcal{F}$. Let $M \in \mathcal{M}_{n}(K)$ have $j$-th column $e_{i}$ and $g \in \mathcal{G}_{1}$. Then

$$
\int^{x_{j}} g\left(x_{j}\right) M^{*}=\gamma_{\mu}^{\prime}\left(1-E_{j}^{*}\right) M^{*} \int^{x_{i}} \gamma[i j]_{\mu}
$$

for $\gamma^{\prime}=\tilde{g}\{j\}^{\prime}, \gamma=\tilde{g}\{j\}$ and $\tilde{g}=g\left[-M_{i, 1}, \ldots,-M_{i, j-1}, 1,-M_{i, j+1}, \ldots,-M_{i, n}\right]$.

Proof. It suffices to consider $i=j=1$ since the general case follows then by multiplying with $(1 j)^{*}$ from the left and $(1 i)^{*}$ from the right. Hence write $M=\left(\begin{array}{ll}1 & v \\ 0 & A\end{array}\right)$ with $v \in K_{n-1}$ and $A \in \mathcal{M}_{n-1}(K)$. Note that $M=\left(\begin{array}{ll}1 & 0 \\ 0 & A\end{array}\right)\left(\begin{array}{ll}1 & v \\ 0 & I\end{array}\right)$ with $I:=I_{n-1}$ so that

$$
\begin{aligned}
\int^{x_{1}} g & \left(x_{1}\right) M^{*}=\int^{x_{1}} g\left(\begin{array}{ll}
1 & v \\
0 & I
\end{array}\right)^{*}\left(\begin{array}{ll}
1 & 0 \\
0 & A
\end{array}\right)^{*}=\int^{x_{1}}\left(\begin{array}{ll}
1 & v \\
0 & I
\end{array}\right)^{*} \tilde{g}\left(\begin{array}{ll}
1 & 0 \\
0 & A
\end{array}\right)^{*}=\left(1-E_{1}^{*}\right)\left(\begin{array}{ll}
1 & v \\
0 & I
\end{array}\right)^{*} \int^{x_{1}} \tilde{g}\left(\begin{array}{ll}
1 & 0 \\
0 & A
\end{array}\right)^{*} \\
& =\left(1-E_{1}^{*}\right)\left(\begin{array}{ll}
1 & v \\
0 & I
\end{array}\right)^{*} \gamma_{\mu}^{\prime} \int^{x_{1}} \gamma_{\mu}\left(\begin{array}{ll}
1 & 0 \\
0 & A
\end{array}\right)^{*}=\left(1-E_{1}^{*}\right)\left(\begin{array}{ll}
1 & v \\
0 & I
\end{array}\right)^{*} \gamma_{\mu}^{\prime}\left(\begin{array}{ll}
1 & 0 \\
0 & A
\end{array}\right)^{*} \int^{x_{1}} \gamma_{\mu} \\
& =\gamma_{\mu}^{\prime}\left(1-E_{1}^{*}\right) M^{*} \int^{x_{1}} \gamma_{\mu},
\end{aligned}
$$

\footnotetext{
3Incidentally, there is a close relation to sumless Sweedler notation: Writing $\gamma:=\Delta^{n} g \in \mathcal{F}_{n}$ for the (iterated) coproduct, one obtains the relation $\Delta^{n} g=\gamma\{1\}_{\mu} \cdots \gamma\{n\}_{\mu}=g_{(1)} \cdots g_{(n)}$ so that one could identify the "formal symbol" $g_{(i)}$ with the component sequence $\gamma\{i\}$.
} 
where the third equality follows from Lemma 2.8, the fourth employs tensor expansion and Item (c) of Lemma 2.7, the fifth follows from $\gamma \in \mathcal{G}_{(1)}^{r}$ and Item (a) of Definition 2.3, the sixth from $\gamma^{\prime} \in \mathcal{G}_{(1)^{\prime}}^{r}$ and straightness.

Lemma 4.1 allows us to put arbitrary one-dimensional integrators $\int^{x_{i}} f\left(x_{i}\right) M^{*}$ into normal form. All we have to do is to apply one sweep of Gaussian elimination to $M$ for creating zeroes underneath the first pivot element in the $j$-th column of $M$. If the pivot is $M_{i j}$ so that $M_{1 j}=\cdots=M_{i-1, j}=0$, one uses the eliminant

$$
L_{i}(-l)=\left(\begin{array}{ccccccc}
1 & & & 0 & & & \\
& \ddots & & \vdots & & & \\
& & 1 & 0 & & & \\
& & & 1 & & & \\
& & & -l_{i+1} & 1 & & \\
& & & & & \ddots & \\
& & & -l_{n} & & & 1
\end{array}\right) \text {, }
$$

where $l=\left(l_{i+1}, \ldots, l_{n}\right) \in K_{n-i}$ with $l_{k}:=M_{k j} / M_{i j}$. Applying this elimination yields

$$
\tilde{M}:=L_{i}(-l) \cdot M=\left(\begin{array}{ccccc}
M_{11} & \cdots & 0 & \cdots & M_{1 n} \\
\vdots & & \vdots & & \ldots \\
M_{i-1,1} & \cdots & 0 & \cdots & M_{i-1, n} \\
M_{i 1} & \cdots & M_{i j} & \cdots & M_{i n} \\
\tilde{M}_{i+1,1} & \cdots & 0 & \cdots & \tilde{M}_{i+1, n} \\
\vdots & & \vdots & & \vdots \\
\tilde{M}_{n 1} & \cdots & 0 & \ldots & \tilde{M}_{n n}
\end{array}\right)
$$

with $M_{i j} e_{i}$ in its $j$-th column.

Proposition 4.2 (Normalization of One-Dimensional Integrators). Let $\left(\mathcal{F}_{n}, \int^{x_{n}}\right)_{n \in \mathbb{N}}$ be a Rota-Baxter hierarchy over a field $K$, and let $\mathcal{G}$ be an admissible coefficient domain for $\mathcal{F}$. Then for $M \in \mathcal{M}_{n}(K)$ and $g \in \mathcal{G}_{1}$ we have

$$
\int^{x_{j}} g\left(x_{j}\right) M^{*}= \begin{cases}M_{i j}^{-1} \gamma_{\mu}^{\prime}\left(1-E_{j}^{*}\right) \tilde{M}^{*} \int^{x_{i}} \gamma\left[d_{j, 1 / M_{i j}}\right]_{\mu} L_{i}(l)^{*} & \text { if } i \neq \infty, \\ \left(\int^{x_{j}} g\left(x_{j}\right)\right) M^{*} & \text { otherwise, }\end{cases}
$$

where $i=\min \left\{k \mid M_{k j} \neq 0\right\}$, and $\tilde{M} \in \mathcal{M}_{n}(K), l \in K_{n-i}$ as in (29), (30) if $i \neq \infty$. Moreover, we set $\gamma^{\prime}=\tilde{g}\{j\}^{\prime}, \gamma=\tilde{g}\{j\}$ and $\tilde{g}=g\left[-\frac{M_{i, 1}}{M_{i j}}, \ldots,-\frac{M_{i, j-1}}{M_{i j}}, 1,-\frac{M_{i, j+1}}{M_{i j}}, \ldots,-\frac{M_{i, n}}{M_{i j}}\right]$.

Proof. Assume first $i=\infty$. Again it suffices to consider $j=1$ since the general case follows by multiplying with $(1 j)^{*}$ from the left. We have to show $\int^{x_{1}} g M^{*} f=\left(\int^{x_{1}} g\right) M^{*} f$ for all $f \in \mathcal{F}$. We may assume $f \in \mathcal{F}_{k}$ for some $k>n$, and we may also view $M \in \mathcal{M}_{k}(K)$. Setting $\tau=(1 k)$, we obtain a matrix $M \tau \in \mathcal{M}_{k}(K)$ with $k$-th column zero, and Item (e) of Lemma 2.7 implies that $(M \tau)^{*} f=\tau^{*} M^{*} f \in \mathcal{F}_{(k)}^{\prime}$. Then we obtain $\int^{x_{k}}\left(\tau^{*} g\right)\left(\tau^{*} M^{*} f\right)=$ 
$\left(\tau^{*} M^{*} f\right)\left(\int^{x_{k}} \tau^{*} g\right)$ from Item (c) of the same lemma. Multiplying this relation by $\tau^{*}$ from the left yields the desired identity.

Now assume the minimum exists. We use the decomposition (30) to obtain

$$
M^{*}=\left(d_{i, 1 / M_{i j}} \tilde{M}\right)^{*} d_{i, M_{i j}}^{*} L_{i}(l)^{*},
$$

such that the $j$-th column of $d_{i, 1 / M_{i j}} \tilde{M}$ is $e_{i}$. Applying Lemma 4.1 and (8) yields

$$
\begin{aligned}
& \int^{x_{j}} g\left(x_{j}\right) M^{*}=\int^{x_{j}} g\left(d_{i, 1 / M_{i j}} \tilde{M}\right)^{*} d_{i, M_{i j}}^{*} L_{i}(l)^{*} \\
& =\gamma_{\mu}^{\prime}\left(1-E_{j}^{*}\right)\left(d_{i, 1 / M_{i j}} \tilde{M}\right)^{*} \int^{x_{i}} \gamma[i j]_{\mu} d_{i, M_{i j}}^{*} L_{i}(l)^{*} \\
& =\gamma_{\mu}^{\prime}\left(1-E_{j}^{*}\right)\left(d_{i, 1 / M_{i j}} \tilde{M}\right)^{*} \int^{x_{i}} d_{i, M_{i j}}^{*} \gamma\left[d_{j, 1 / M_{i j}}\right]_{\mu} L_{i}(l)^{*} \\
& =M_{i j}^{-1} \gamma_{\mu}^{\prime}\left(1-E_{j}^{*}\right)\left(d_{i, 1 / M_{i j}} \tilde{M}\right)^{*} d_{i, M_{i j}}^{*} \int^{x_{i}} \gamma\left[d_{j, 1 / M_{i j}}\right]_{\mu} L_{i}(l)^{*} \\
& =M_{i j}^{-1} \gamma_{\mu}^{\prime}\left(1-E_{j}^{*}\right) \tilde{M}^{*} \int^{x_{i}} \gamma\left[d_{j, 1 / M_{i j}}\right]_{\mu} L_{i}(l)^{*}
\end{aligned}
$$

which is indeed (31) for $i \neq \infty$.

Before we proceed to the ordering of one-dimensional integrators, let us note the following natural commutativity result for integrals and evalutions along distinct axes.

Corollary 4.3. For $i \neq j$ the operators $\int^{x_{i}}$ and $E_{j}^{*}$ commute.

Proof. Applying Lemma 4.1 with $M=E_{j}$ and $g=1$ yields $\int^{x_{i}} E_{j}^{*}=\left(1-E_{i}^{*}\right) E_{j}^{*} \int^{x_{i}}$, which implies commutativity because of $E_{i} E_{j}=E_{j} E_{i}$ and (f) of Lemma 2.7.

By Proposition 4.2, the basic building blocks are one-dimensional inegrators of the form $\int^{x_{i}} h\left(x_{i}\right) L_{i}(v)^{*}$ along a coordinate axis $x_{i}$. We shall now investigate how two such integrators interact with each other, starting with the case of different coordinate axes. It turns out that we can always reorder them. In this paper, we choose to put them in ascending order.

Proposition 4.4 (Ordering of One-Dimensional Integrators).

Let $\left(\mathcal{F}_{n}, \int^{x_{n}}\right)_{n \in \mathbb{N}}$ be a Rota-Baxter hierarchy over a field $K$, and $\mathcal{G}$ be an admissible coefficient domain for $\mathcal{F}$. Then for $i<j$, vectors $v \in K^{n-i}, w \in K^{n-j}$ and functions $g, h \in \mathcal{G}_{1}$ we have

$$
\int^{x_{j}} h\left(x_{j}\right) L_{j}(w)^{*} \int^{x_{i}} g\left(x_{i}\right) L_{i}(v)^{*}=\left(1-E_{j}^{*}\right) \int^{x_{i}} \eta_{\mu}^{\prime} L_{i}\left(v^{\prime}\right)^{*} \int^{x_{j}} \eta_{\mu} L_{j}(w)^{*},
$$

where we set $v^{\prime}=L_{j-i}^{-1}(w) v=\left(v_{i+1}, \cdots, v_{j}, v_{j+1}-w_{j+1} v_{j}, \ldots, v_{n}-w_{n} v_{j}\right)^{\top} \in K^{n-i}$, as well as $\eta^{\prime}=g\left(x_{i}\right) \tilde{h}\{i\}, \eta=\tilde{h}\{j\}$ and $\tilde{h}=h\left[e_{j}-v_{j} e_{i}\right] \in \mathcal{G}_{(i, j)}$.

Proof. First note that Lemma 4.1 with $g=1$ yields $\int^{x_{i}} L_{j}(w)^{*}=L_{j}(w)^{*} \int^{x_{i}}-\left(L_{j}(w) E_{i}\right)^{*} \int^{x_{i}}$, but the last summand vanishes by (f) of Lemma 2.7 since $L_{j}(w) E_{i}=E_{i} L_{j}(w)$. Hence

$$
\begin{array}{rl}
\int^{x_{j}} & h\left(x_{j}\right) L_{j}(w)^{*} \int^{x_{i}} g\left(x_{i}\right) L_{i}(v)^{*}=\int^{x_{j}} h\left(x_{j}\right) \int^{x_{i}} L_{j}(w)^{*} g\left(x_{i}\right) L_{i}(v)^{*} \\
& =\int^{x_{i}} g\left(x_{i}\right) \int^{x_{j}} h\left(x_{j}\right) L_{i}\left(v^{\prime}\right)^{*} L_{j}(w)^{*}=\int^{x_{i}} g\left(x_{i}\right) \tilde{h}\{i\}_{\mu}\left(1-E_{j}^{*}\right) L_{i}\left(v^{\prime}\right)^{*} \int^{x_{j}} \tilde{h}\{j\}_{\mu} L_{j}(w)^{*} \\
& =\left(1-E_{j}^{*}\right) \int^{x_{i}} g\left(x_{i}\right) \tilde{h}\{i\}_{\mu} L_{i}\left(v^{\prime}\right)^{*} \int^{x_{j}} \tilde{h}\{j\}_{\mu} L_{j}(w)^{*}
\end{array}
$$


where the second step follows from (4) and Item (c) of Lemma 2.7, from $L_{j}(w)_{\lrcorner i}=I_{i}$ and the commutativity of Rota-Baxter operators, the third from Lemma 4.1 with $\tilde{h}=L_{i}\left(v^{\prime}\right)_{j \bullet}^{*} h$ and $L_{i}\left(v^{\prime}\right)_{j \bullet}=-v_{j}^{\prime} e_{i}+e_{j}=e_{j}-v_{j} e_{j}$, the last step from Corollary 4.3 and straightness.

Lemma 4.5. Let $\left(\mathcal{F}_{n}, \int^{x_{n}}\right)_{n \in \mathbb{N}}$ be a Rota-Baxter hierarchy over a field $K$, and $\mathcal{G}$ an admissible coefficient domain for $\mathcal{F}$. Then for $i \in \mathbb{N}, h \in \mathcal{G}_{1}$ and $w \in K^{n-i} \backslash\{0\}$ we have

$$
\int^{x_{i}} h\left(x_{i}\right) L_{i}(w)^{*} \int^{x_{i}}=L_{k}^{-1}\left(w^{\prime}\right)^{*} \eta^{\prime}[k n+1]_{\mu}\left(L_{i}(\bar{w})^{*} \int^{x_{i}}-\int^{x_{i}} L_{i}(\bar{w})^{*}\right) \int^{x_{k}} \eta_{\mu} L_{k}\left(w^{\prime}\right)^{*}
$$

as an identity on $\mathcal{F}_{n}$, where $k=\min \left\{j>i \mid w_{j} \neq 0\right\}$ and $\eta^{\prime}=\tilde{h}\{n+1\}, \eta=\tilde{h}\{k\}$ with

$$
\tilde{h}:=h\left[e_{k} / w_{k}-e_{n+1} / w_{k}\right] / w_{k} \in \mathcal{F}_{(k, n+1)} .
$$

Moreover, for $w=\left(w_{i+1}, \ldots, w_{n}\right)^{\top}$ we have defined

$$
\bar{w}=w_{k} e_{k-i} \in K^{n-i}, \quad w^{\prime}=\left(w_{k+1}, \ldots, w_{n}\right) / w_{k} \in K^{n-k}
$$

as auxiliary vectors.

Proof. It suffices to assume $i=1$; the general case follows by conjugation with $\tau^{*}=(i 1)^{*}$ since $\tau L_{k}\left(w^{\prime}\right) \tau=L_{k}\left(w^{\prime}\right)$ and $\tau L_{1}(\bar{w}) \tau=L_{i}(\bar{w})$. We write $J:=I_{n} \oplus e_{k}$ as an abbreviation. Using the relation $L_{x}(w)^{*}=w_{k}^{*} L_{x}\left(e_{k-1}+\left(0, w^{\prime}\right)^{\top}\right)\left(1 / w_{k}\right)^{*}$, we obtain

$$
\begin{aligned}
& \int^{x} h(x) L_{x}(w)^{*} \int^{x}=w_{k}^{-2} w_{k}^{*} \int^{x} h\left[d_{1,1 / w_{k}}\right] L_{x}\left(e_{k-1}+\left(0, w^{\prime}\right)^{\top}\right)^{*} \int^{x}\left(\frac{1}{w_{k}}\right)^{*} \\
& =w_{k}^{-1} w_{k}^{*} L_{k}^{-1}\left(w^{\prime}\right)^{*} J^{*}\left(L_{x}\left(e_{k-1}\right)^{*} \int^{x}-\int^{x} L_{x}\left(e_{k-1}\right)^{*}\right) \int^{x_{k}} \tilde{h} L_{k}\left(w^{\prime}\right)^{*}\left(\frac{1}{w_{k}}\right)^{*} \\
& =w_{k}^{-1} L_{k}^{-1}\left(w^{\prime}\right)^{*} J^{*}\left(w_{k}^{*} L_{x}\left(e_{k-1}\right)^{*} \int^{x}-w_{k}^{*} \int^{x} L_{x}\left(e_{k-1}\right)^{*}\right) \int^{x_{k}} \tilde{h} L_{k}\left(w^{\prime}\right)^{*}\left(\frac{1}{w_{k}}\right)^{*} \\
& =L_{k}^{-1}\left(w^{\prime}\right)^{*} J^{*}\left(L_{x}(\bar{w})^{*} \int^{x}-\int^{x} L_{x}(\bar{w})^{*}\right) \int^{x_{k}} \tilde{h} w_{k}^{*} L_{k}\left(w^{\prime}\right)^{*}\left(\frac{1}{w_{k}}\right)^{*} \\
& =L_{k}^{-1}\left(w^{\prime}\right)^{*} J^{*}\left(L_{x}(\bar{w})^{*} \int^{x}-\int^{x} L_{x}(\bar{w})^{*}\right) \int^{x_{k}} \tilde{h} L_{k}\left(w^{\prime}\right)^{*}
\end{aligned}
$$

where the first equality uses (8) twice, the second employs (13), the third the commutation of $w_{k} e_{11}+e_{22}+\cdots e_{n n}$ with $L_{k}^{-1}\left(w^{\prime}\right)$ and $J$, the fourth applies again (8) together with $w_{k}^{*} L_{x}\left(e_{k-1}\right)^{*}=L_{x}(\bar{w})^{*} w_{k}^{*}$, and the last $w_{k}^{*} L_{k}\left(w^{\prime}\right)^{*}\left(1 / w_{k}\right)^{*}=L_{k}\left(w^{\prime}\right)^{*}$. Finally, (33) follows by substituting the expansion of $\tilde{h}$, noting that the components $\eta^{\prime}=\tilde{h}\{n+1\} \in \mathcal{F}_{(n+1)}^{r}$ commute with $\int^{x}$ and $L_{x}(\bar{w})^{*}$ and that $J^{*}$ acts on them as $(k n+1)^{*}$.

For stating the last operator relation, we need the following technical result for composing two substitutions. A general result could be formulated but for our present purposes the following special case will be sufficient, where the first substitution is $x_{1} \mapsto a x_{k}+b x_{l}$, and the second is $x_{k} \mapsto c x_{i}+d x_{j}$.

Lemma 4.6. Let $\left(\mathcal{G}_{n}, \int^{x_{n}}\right)_{n \in \mathbb{N}}$ be a separated Rota-Baxter hierarchy over $K$. Fix $h \in \mathcal{G}_{1}$, indices $i<j \leq k<l$, and scalars $a, b, c, d \in K$. Assume $h\left[a e_{k}+b e_{l}\right]=u$ is expanded into $u=\eta_{\mu}^{\prime} \eta_{\mu}$ with $\eta=u\{k\} \in \mathcal{G}_{(k)}^{r}$ and $\eta^{\prime}=u\{l\} \in \mathcal{G}_{(l)}^{r}$. Setting $\eta_{\mu}=u_{\mu}\left(x_{k}\right)$, expand 
also $u_{\mu}\left[c e_{i}+d e_{j}\right]=\tilde{u}_{\mu}$ with $\gamma_{\mu}^{\prime}=\tilde{u}_{\mu}\{j\} \in \mathcal{G}_{(j)}^{s_{\mu}}$ and $\gamma_{\mu}=\tilde{u}\{i\} \in \mathcal{G}_{(i)}^{s_{\mu}}$ into $\tilde{u}_{\mu}=\gamma_{\mu \nu}^{\prime} \gamma_{\mu \nu}$, where summation is only over $\nu$. Then in $\mathcal{G}_{(i, j, l)}$ we have the standard tensor expasion

$$
h\left[\text { ace }_{i}+a d e_{j}+b e_{l}\right]=\eta_{\mu}^{\prime} \gamma_{\mu \nu}^{\prime} \gamma_{\mu \nu}
$$

with the sum over $(\mu, \nu) \in\left\{1, \ldots, s_{1}\right\} \times \cdots \times\left\{1, \ldots, s_{r}\right\} \cong\left\{1, \ldots, s_{1}+\cdots+s_{r}\right\}$.

Proof. Choosing $n \geq l$, write $M=a e_{k}+b e_{l} \in \mathcal{M}_{n}(K)$ and $\tilde{M}=I_{k-1} \oplus\left(c e_{i}+d e_{j}\right) \in \mathcal{M}_{n}(K)$ in the sense of the embedding. Operating $\tilde{M}^{*}$ on $h[M]=\eta_{\mu}^{\prime} \eta_{\mu}$ yields

$$
h\left[a_{c} e_{i}+a d e_{j}+b e_{l}\right]=h[M \tilde{M}]=\eta_{\mu}^{\prime} \eta_{\mu}[\tilde{M}]
$$

since $\tilde{M}^{*}$ is an algebra homomorphism which leaves $\eta_{\mu}^{\prime} \in \mathcal{G}_{(l)}$ invariant (this follows easily from straightness and $\left.\tilde{M}_{l \bullet}=e_{l}\right)$. But $u_{\mu}=\eta_{\mu}[1 k]$ implies $\tilde{u}_{\mu}=\eta_{\mu}[\tilde{M}]$ since the matrices $\tilde{M}$ and $(1 k)\left(\left(c e_{i}+d e_{j}\right) \oplus I_{n-1}\right)$ have the same $k$-th row (again the conclusion follows by straightness). Now (34) follows by substituting the tensor expansion of $\tilde{u}_{\mu}$.

We can now state the last important relation, which will enable us to put one-dimensional integrators into strictly ascending order since two adjacent integrators along the same coordinate axes can be reduces as the following propostion makes precise.

Proposition 4.7 (Coalescence of One-Dimensional Integrators). Let $\left(\mathcal{F}_{n}, \int^{x_{n}}\right)_{n \in \mathbb{N}}$ be a Rota-Baxter hierarchy over a field $K$, and $\mathcal{G}$ an admissible coefficient domain for $\mathcal{F}$. Then for $i \in \mathbb{N}$, and $v, w \in K^{n-i}$ with $w \neq 0$ we have on $\mathcal{F}_{n}$ the identity

$$
\begin{aligned}
& \int^{x_{i}} h\left(x_{i}\right) L_{i}(w)^{*} \int^{x_{i}} g\left(x_{i}\right) L_{i}(v)^{*}=L_{k}^{-1}\left(w^{\prime}\right)^{*} \chi^{\prime \prime}[k n+1]_{\mu} \times \\
& \quad \times\left(L_{i}(\bar{w})^{*} \int^{x_{i}} g\left(x_{i}\right) \chi_{\mu}^{\prime} L_{i}\left(v^{\prime}\right)^{*}-\int^{x_{i}} g\left(x_{i}\right) \chi_{\mu}^{\prime} L_{i}\left(v^{\prime}+\bar{w}\right)^{*}\right) \int^{x_{k}} \chi_{\mu} L_{k}\left(w^{\prime}\right)^{*},
\end{aligned}
$$

where we have set

$$
\begin{aligned}
\tilde{h} & =h\left[-\left(v_{k}^{\prime} / w_{k}\right) e_{i}+e_{j} / w_{k}-e_{n+1} / w_{k}\right] \\
v^{\prime} & =L_{k-i}^{-1}\left(w^{\prime}\right) v=\left(v_{i+1}, \ldots, v_{k}, v_{k+1}-w_{k+1}^{\prime} v_{k}, \ldots, v_{n}-w_{n}^{\prime} v_{k}\right)^{\top} \in K^{n-i} .
\end{aligned}
$$

with component vectors $\chi^{\prime \prime}=\tilde{h}\{n+1\}, \chi^{\prime}=\tilde{h}\{i\}, \chi=\tilde{h}\{k\}$, and $w^{\prime}, \bar{w}$ from Lemma 4.5. Proof. Multiplying (33) by $g\left(x_{i}\right) L_{i}(v)^{*}$ from the right, the right-hand side of (35) becomes

$$
L_{k}^{-1}\left(w^{\prime}\right)^{*} \eta^{\prime}[k n+1]_{\mu}\left(L_{i}(\bar{w})^{*} \int^{x_{i}}-\int^{x_{i}} L_{i}(\bar{w})^{*}\right) \int^{x_{k}} \eta_{\mu} L_{k}\left(w^{\prime}\right)^{*} g\left(x_{i}\right) L_{i}(v)^{*} .
$$

Since $L_{k}\left(w^{\prime}\right)^{*}$ commutes with $g\left(x_{i}\right)$ and (4) swaps eliminants, the right-hand factor is

$$
\begin{gathered}
\int^{x_{k}} \eta_{\mu} L_{k}\left(w^{\prime}\right)^{*} g\left(x_{i}\right) L_{i}(v)^{*}=g\left(x_{i}\right) \int^{x_{k}} \eta_{\mu} L_{i}\left(v^{\prime}\right)^{*} L_{k}\left(w^{\prime}\right)^{*} \\
=g\left(x_{i}\right) \gamma_{\mu \nu}^{\prime}\left(1-E_{k}^{*}\right) L_{i}\left(v^{\prime}\right)^{*} \int^{x_{k}} \gamma_{\mu \nu} L_{k}\left(w^{\prime}\right)^{*}
\end{gathered}
$$

where the last expression (summation only over $\nu$ ) follows from Lemma 4.1 by writing $\eta_{\mu}=u_{\mu}\left(x_{k}\right)$ and defining $\tilde{u}_{\mu}=u_{\mu}\left[e_{k}-v_{k}^{\prime} e_{i}\right]$ with component vectors $\gamma_{\mu}^{\prime}=\tilde{g}\{i\}, \gamma_{\mu}=\tilde{g}\{k\}$. By Lemma 34 we can determine $s \in \mathbb{N}$ and make the identifications $\eta^{\prime}=\chi^{\prime \prime} \in \mathcal{G}_{(n+1)}^{s}$, 
$\gamma^{\prime}=\chi^{\prime} \in \mathcal{G}_{(i)}^{s}$, and $\chi=\gamma \in \mathcal{G}_{(k)}^{s}$. Comparing the above equations with (35) and noting that $g\left(x_{i}\right) \chi_{\mu}^{\prime} \in \mathcal{G}_{(i)}$ commutes with $L_{i}(\bar{w})^{*}$, it is clear that it suffices now to prove that

$$
\left(L_{i}(\bar{w})^{*} \int^{x_{i}}-\int^{x_{i}} L_{i}(\bar{w})^{*}\right) g\left(x_{i}\right) \chi_{\mu}^{\prime} E_{k}^{*}=\left(L_{i}(\bar{w})^{*} \int^{x_{i}} E_{k}^{*}-\int^{x_{i}} L_{i}(\bar{w})^{*} E_{k}^{*}\right) g\left(x_{i}\right) \chi_{\mu}^{\prime}
$$

vanishes, where we have used that $g\left(x_{i}\right) \chi_{\mu}^{\prime} E_{k}^{*}=E_{k}^{*} g\left(x_{i}\right) \chi_{\mu}^{\prime}$ by $g\left(x_{i}\right) \chi_{\mu}^{\prime} \in \mathcal{G}_{(i)}$. But the parenthesis on the right-hand side above is zero by $L_{i}(\bar{w})^{*} E_{k}^{*}=E_{k}^{*}$ and Corollary 4.3 .

We have now collected all essential operator relations for building up the partial integral operators as a quotient ring modulo these relations.

4.2. Construction via Quotient. The raw material for building the operator ring over an admissible coefficient domain $\mathcal{G}$ for some Rota-Baxter hierarchy $\mathcal{F}$ comes from the three base algebras: the coefficient algebra $\mathcal{G}$; the substitutions from $K[\mathcal{M}]$ in the form of a monoid algebra, and the commuting Rota-Baxter operators (a polynomial ring in infinitley many variables). These three algebras are put together by a free product, then we take a suitable quotient modulo the ideal of operator relations.

We denote the free product $[10, \S$ III.6] [16, §29] [23, p. 85] of two $K$-algebras $A$ and $B$ by $A \amalg_{K} B$. Rougly speaking, this consists of $K$-linear combinations of alternating products of elements of $A$ and $B$; for a short introduction see also [15, Example 3.28] where $K$ is the complex field (though the construction works for general fields) or the historical reference [22]. One has natural isomorphisms $A \amalg_{K}\left(B \amalg_{K} C\right) \cong\left(A \amalg_{K} B\right) \amalg_{K} C$ so that we may employ the notation $A \amalg_{K} B \amalg_{K} C$. Incidentally, note that the free product is commutative in the sense that $A \amalg_{K} B \cong B \amalg_{K} A$, despite having a noncommutative multiplication.

Recall that $\mathcal{M}:=\mathcal{M}(K)$ denotes the substitution monoid over $K$, which contains the semigroup $\mathcal{M}^{\times}=\mathcal{M}(K) \backslash\{I\}$. Since its action on $\mathcal{F}$ and $\mathcal{G} \leq \mathcal{F}$ is contravariant, we form the opposite monoid algebra $K[\mathcal{M}]^{*}$ and the associated opposite semigroup algebra $K\left[\mathcal{M}^{\times}\right]^{*}$. Its basis elements will be written as $M^{*}$ with $M \in K[\mathcal{M}]$. For avoiding confusion, we shall write the integrals in the prospective operator ring as $A_{n}(n \in \mathbb{N})$; they form the commutative polynomial ring $K[A]=K\left[A_{n} \mid n>0\right]$. After setting up an appropriate action $\odot$ on the given Rota-Baxter hierarchy $\left(\mathcal{F}_{n}, \int^{x_{n}}\right)_{n \in \mathbb{N}}$, we shall have $A_{n} \odot f=\int^{x_{n}} f$ and $M^{*} \odot f=f[M]$ and $f \odot g=f g$.

Definition 4.8. Let $\left(\mathcal{G}, \int\right)$ be a separated Rota-Baxter hierarchy over a field $K$. Then the ring of partial integral operators over $\mathcal{G}$ is defined as the quotient $K$-algebra

$$
\mathcal{G}\left[\int\right]:=\mathcal{G} \amalg_{K} K[\mathcal{M}]^{*} \amalg_{K} K[A] / \cong
$$

with the congruence $\cong$ generated by Table 1 for the implicit ranges $i, j \in \mathbb{N}$ such that $i<j$ and $M \in K[\mathcal{M}]$, and $g, h \in \mathcal{G}_{1}$. For all other notations and conditions, we refer to the Propositions 4.2, 4.4, and 4.7.

Note that Table 1 is meant to contain various implied congruences as special instances of the ones given, obtained by substituting $g\left(x_{k}\right)=1$ in the first rule; $g\left(x_{j}\right)=1$ in the rules $4,5,6$; and $h\left(x_{j}\right)=1$ or $g\left(x_{i}\right)=1$ or both in the last two rules. Moreover, $v=0$ and 


\begin{tabular}{|ll|}
\hline$M^{*} g \cong g[M] M^{*}$ & $M^{*} A_{i} \cong 0 \quad$ if $M_{i \bullet}=0$ \\
$A_{j} g\left(x_{i}\right) \cong g\left(x_{i}\right) A_{j}$ & $A_{i} g\left(x_{j}\right) \cong g\left(x_{j}\right) A_{i}$ \\
$A_{j} g\left(x_{j}\right) M^{*} \cong M_{i j}^{-1} \gamma_{\mu}^{\prime}\left(1-E_{j}^{*}\right) \tilde{M}^{*} \int^{x_{i}} \gamma\left[d_{j, 1 / M_{i j}}\right]_{\mu} L_{i}(l)^{*}$ & if $i:=\min \left\{k \mid M_{k j} \neq 0\right\} \neq \infty$ \\
$A_{j} g\left(x_{j}\right) M^{*} \cong\left(\int^{x_{j}} g\left(x_{j}\right)\right) M^{*}$ & if $i:=\min \left\{k \mid M_{k j} \neq 0\right\}=\infty$ \\
$A_{j} h\left(x_{j}\right) L_{j}(w)^{*} A_{i} g\left(x_{i}\right) L_{i}(v)^{*} \cong\left(1-E_{j}^{*}\right) A_{i} \eta_{\mu}^{\prime} L_{i}\left(v^{\prime}\right)^{*} A_{j} \eta_{\mu} L_{j}(w)^{*}$ \\
$A_{i} h\left(x_{i}\right) L_{i}(w)^{*} A_{i} g\left(x_{i}\right) L_{i}(v)^{*} \cong L_{k}^{-1}\left(w^{\prime}\right)^{*} \chi^{\prime \prime}[k n+1]_{\mu} \times$ \\
$\quad \times\left(L_{i}(\bar{w})^{*} \int^{x_{i}} g\left(x_{i}\right) \chi_{\mu}^{\prime} L_{i}\left(v^{\prime}\right)^{*}-\int^{x_{i}} g\left(x_{i}\right) \chi_{\mu}^{\prime} L_{i}\left(v^{\prime}+\bar{w}\right)^{*}\right) \int^{x_{k}} \chi_{\mu} L_{k}\left(w^{\prime}\right)^{*}$ \\
$A_{j} g\left(x_{j}\right) A_{j} \cong\left(\int^{x_{j}} g\left(x_{j}\right)\right) A_{j}-A_{j}\left(\int^{x_{j}} g\left(x_{j}\right)\right)$ & \\
\hline
\end{tabular}

TABLE 1. Relations for Partial Integral Operators and Substitutions

hence $L_{i}(v)=I$ is allowed in the last rule. These implicit rewrite rules are also important for the implementation.

The definition of the free product $H=H_{1} \amalg_{K} \cdots \amalg_{K} H_{k}$ yields the coproduct in the category of $K$-algebras, with insertions $H_{k} \hookrightarrow H$. This requires for each factor a unitary splitting $H_{i} \cong K \oplus \tilde{H}_{i}$ as $K$-vector spaces such that the alternating products are built from $\tilde{H}_{i}$ rather than $H_{i}$, with $1_{H}$ being the empty product. In our case, the implicit rules will remove the units $1_{\mathcal{G}} \in \mathcal{G}$ and $I^{*} \in K[\mathcal{M}]^{*}$ and $1_{K[A]} \in K[A]$ in favor of the new unit in $K \subset \mathcal{G}\left[\int\right]$. We use the unitary splittings

$$
\begin{aligned}
\mathcal{G} & =K \oplus \cup_{n>0} \mathcal{G}_{n}, \\
K[\mathcal{M}]^{*} & =K \oplus K\left[\mathcal{M}^{\times}\right]^{*}, \\
K[A] & =K \oplus\{p \in K[A] \mid \operatorname{deg}(p)>0\}
\end{aligned}
$$

for the three factors in the free product (36).

A detailed description of the computational realization of $\mathcal{G}\left[\int\right]$ will be the subject of another paper. For our present purposes it suffices to observe that we can start from the free $K$-algebra generated by a basis $\left(b_{i}\right)_{i \in \mathbb{N}}$ of $\mathcal{G}$ such that $b_{0}=1$, the substitutions $M^{*} \in K[\mathcal{M}]$ and the integrators $\left(A_{n}\right)_{n>0}$. Then one has the following extra rules in addition to the explicit and implicit rules of Table 1:

- Every occurrence of $b_{0}, I^{*}$, and $1_{K[A]}$ is removed, while the empty word is treated as the true unit $1 \in \mathcal{G}\left[\int\right]$.

- After operating on a basis function $b_{i} \in \mathcal{G}$ by multiplication, integration or substitution, the resulting element of $\mathcal{G}$ is again expanded with respect to the basis $\left(b_{i}\right)_{i \in \mathbb{N}}$.

- Within monomials of $K[A]$, one enforces the ordering $A_{1}>A_{2}>\cdots$, thus achieving normal forms $A^{\alpha}=A_{1}^{\alpha_{1}} A_{2}^{\alpha_{2}} \cdots$ for $\alpha: \mathbb{N}^{\times} \rightarrow \mathbb{N}$. In view of Table 1 , reduction ensures $\alpha_{i} \in\{0,1\}$. 
For the subtle interplay between canonical forms and basis expansion, we refer to [37, §4.4], where this is discussed for ordinary integro-differential operators (but the same applies here mutatis mutandis).

Proposition 4.9. Let $\left(\mathcal{F}_{n}, \int^{x_{n}}\right)_{n \in \mathbb{N}}$ be a Rota-Baxter hierarchy over a field $K$, and let $\mathcal{G}$ be an admissible coefficient domain for $\mathcal{F}$. Then the action $\odot: \mathcal{G}\left[\int\right] \times \mathcal{F} \rightarrow \mathcal{F}$ induced by $g \odot f=g f, M^{*} \odot f=f[M]$ and $A_{i} \odot f=\int^{x_{i}} f$ is well-defined.

Proof. As indicated, one may interpret $\mathcal{G}\left[\int\right]$ as a quotient of the free $K$-algebra generated by a $K$-basis $\left(b_{i}\right)_{i \in \mathbb{N}}$ of $\mathcal{G}$, the substitutions $M^{*} \in K[\mathcal{M}]$ and the integrators $\left(A_{n}\right)_{n>0}$. Since the implicit rules and the three extra ones listed above are obvious consequences, it suffices to verify Table 1 for basis functions $g, g_{j}, g_{k}$. Rule 1 is valid because $K[\mathcal{M}]$ acts on $\mathcal{G} \subseteq \mathcal{F}$ via $K$-algebra homomorphisms, by hypothesis (Definition 2.1). Rule 2 follows from $\bar{M}=E_{i} M$ and Item (f) of Lemma 2.7, Rules 3, 4 by Item (c). Rules 5, 6 correspond to Proposition 4.2, Rule 7 to Proposition 4.4, Rule 8 to Proposition 4.7. Finally, Rule 9 follws since each $\left(\mathcal{G}_{n}, \int^{x_{n}}\right)$ is a Rota-Baxter algebra in its own right.

We orient the congruences of Table 1 from left to right. Moreover, we agree that on the right-hand side all matrix products, substitutions and coefficient multiplications are immediately carried out. For example, the result of Rule 3 is a $K$-linear combination of monomials having the form $\mathcal{G} \cdot K[\mathcal{M}]^{*} \cdot A_{i} \cdot \mathcal{G}_{(i)} \cdot K[\mathcal{M}]^{*}$. For showing termination of the induced rewrite system (including the implicit and extra rules), we use the following term order on the underlying free algebra. Every monomial is segmented into $W=w_{0} A_{i_{1}} w_{1} A_{i_{2}} \cdots A_{i_{n}} w_{n}$ such that the words $w_{0}, w_{1}, \ldots, w_{n}$ do not contain any $A$. Note that every word $w_{k}$ with $k>0$ has a corresponding integration index $i_{k}$, and by convention we set $i_{0}:=0$. For any subword $w_{k}$, let $\sigma\left(w_{k}\right)$ denote $w_{k}$ viewed as an element in the following Noetherian partial order on the word monoid over the alphabet $\mathcal{G} \uplus K[\mathcal{M}]^{*}$. We impose no order amongst the $g \in \mathcal{G}$ or amongst the $M^{*} \in K[\mathcal{M}]^{*}$ but we stipulate that $g<M^{*}$ whenever $M \neq I$; this partial order on the alphabet is extended to a graded lexicographic partial order on the corresponding words. Furthermore, let $N\left(w_{k}\right)$ be the total number of nonzero matrix entries $M_{r, i_{k}}$ with $r<i_{k}$ and nonunit matrix entries $M_{i_{k}, i_{k}}$, for all $M^{*}$ occurring in the word $w_{k}$. If $u_{l}$ is another word, we put now $w_{k}<u_{l}$ iff $\left(N(w),-i_{k}, \sigma\left(w_{k}\right)\right)<\left(N(u),-j_{l}, \sigma\left(u_{l}\right)\right)$ in the pure lexicographic sense. Finally, let $U=u_{0} A_{j_{1}} u_{1} A_{j_{2}} \cdots A_{j_{m}} u_{m}$ be a second monomial in the free algebra. Then we define $W<U$ iff $\left(w_{n}, \ldots, w_{1}, w_{0}\right)<\left(u_{m}, \ldots, u_{1}, u_{0}\right)$ in the sense of the graded lexicographic order induced by the previous order given on the components.

Theorem 4.10. Let $\left(\mathcal{G}, \int\right)$ be a separated Rota-Baxter hierarchy over a field $K$. Orienting the rules of Table 1 from left to right, one obtains a Noetherian reduction system.

Proof. We note first that all the rules of Table 1 respect the above term order in the sense that all monomials on the right-hand side are smaller than the monomial on the left-hand side. For Rules $1-4$ this is evident. Rule 5 is only applicable if $M$ does not have the form $L_{j}(v)$ so that $N(w)>0$ for the word on the left-hand side while clearly $N(u)=0$ for all rightmost words of the right-hands side monomials. In Rule 6 we have a drop in the 
number of integrators, so the left-hand side is greater due to our choice of graded order on subword sequences. Looking at Rule 7 we see that the last subword $w$ has $N(w)=0$ before and after reduction but its negated integration index drops from $-i$ to $-j$. The same is true for Rule 8, where the index drops from $-i$ to $-k$. Finally, Rule 9 decreases the number of integrators and hence drops in the graded order on subword sequences.

Next let us convince ourselves that $>$ is a term order. If 1 denotes the empty word, we have $1<W$ for all non-empty words. This is because the empty word has $N(1)=0$ and integration index 0 , and $\sigma(1)$ is the mimimum among all $\sigma$-words. Now we assume $W<U$ and we must show $W V<U V$ and $V W<V U$ for all words $V$ in the free algebra. But this is clear since multiplication by $V$ on either side equally affects $N(w)$ and the integration index of $w$ of each segmental word $w$ within $V$, and the order on the $\sigma(w)$ respects concatenation.

It remains to prove that $>$ is Noetherian. By way of contradiction, assume an infinite descending chain exists. Since $>$ is graded on subword sequences, there must then be an infinite descending chain of words $w_{0} A_{i_{1}} w_{1} A_{i_{2}} \cdots A_{i_{n}} w_{n}$ with fixed sequence length $n$. Since the $N\left(w_{k}\right)$ and the integration indices cannot drop infinitely often, we obtain an infinite descending chain of $\sigma\left(w_{k}\right)$ instances. But this is impossible because the order on the $\sigma\left(w_{k}\right)$ is Noetherian.

In a Noetherian reduction system, every element has at least one normal form. Each normal form may be taken to be a representative of the corresponding congruence class of the quotient algebra (36). In our case, we can go one step further: We can specify monomial normal forms, such that every element of the quotient algebra can be written as a $K$-linear combination of these (congruence classes of) monomomial normal forms. This can be done as follows.

Let us call a word (of the underlying free algebra) a line integrator of index $i$ if is has the form $A_{i} b\left(x_{i}\right) L_{i}(v)^{*}$ with a $K$-basis element $b \in \mathcal{G}_{1}$, where again $b=1$ or $L_{i}(v)=I$ means absence. A volume integrator is word of the form $K=b M^{*} J_{1} \cdots J_{r}$, where $b \in \mathcal{G}$ is a basis element and $M^{*} \in K[\mathcal{M}]^{*}$ with $M_{i_{1}} \bullet \neq 0$ for $r>0$, and where $J_{1}, \ldots, J_{r}$ are line integrators of indices $i_{1}<\cdots<i_{r}$.

Theorem 4.11. Let $\left(\mathcal{G}, \int\right)$ be a separated Rota-Baxter hierarchy over a field $K$. Then every element of $\mathcal{G}\left[\int\right]$ is a K-linear combination of terms $b M^{*} J_{1} \cdots J_{r}$, where $b \in \mathcal{G}$ is a basis element and $M^{*} \in K[\mathcal{M}]^{*}$ with $M_{i_{1}} \bullet \neq 0$ for $r>0$, and where $J_{1}, \ldots, J_{r}$ are integrators of indices $i_{1}<\cdots<i_{r}$.

Proof. We have to show that every word can be reduced to a linear combination of the form stated in the Theorem. Using Rule 1 will ensure all subwords $w_{k}$ in the canonical segmentation $w_{0} A_{i_{1}} w_{1} A_{i_{2}} \cdots A_{i_{n}} w_{n}$ have the form $b_{k} M_{k}^{*}$ for some $b_{k} \in \mathcal{G}$ and $M_{k} \in K[\mathcal{M}]$. By Rules 3 and 4 we can achieve that $b_{k} \in \mathcal{G}_{\left(i_{k}\right)}$ and by Rules $5 / 6$ that $M_{k}=L_{i_{k}}(v)^{*}$ for some $v$; again $v=0$ means absence. Hence all monomials can be reduced to the form $b M^{*} J_{1} \cdots J_{r}$, where $b \in \mathcal{G}$ is any $K$-basis elements and $M^{*} \in K[\mathcal{M}]^{*}$ is arbitrary. If the integration index drops between any neighboring integrators $J_{i}, J_{i+1}$, application of Rule 7 fixes this. Hence we can ensure that the sequence of integration indices is increasing. 
For achieving a stricly increasing sequence, it remains to apply Rule 8 if $w \neq 0$ and Rule 9 otherwise. Finally, Rule 2 is used to guarantee $M_{i_{1}} \bullet \neq 0$ if $r>0$.

In fact, it is our conjecture that the reduction system induced by Table 1 (or perhaps a slight variation of it) is not only Noetherian but also confluent (modulo the $K$-algebra axioms, as usual). In other words, we have a noncommutative Gröbner basis for the relation ideal defined by the noncommutative polynomials corresponding to these rules. As a consequence, the volume integrators would be a $K$-basis of $\mathcal{G}\left[\int\right]$.

Conjecture 4.12. Let $\left(\mathcal{G}, \int\right)$ be a separated Rota-Baxter hierarchy over a field K. Orienting the rules of Table 1 from left to right, one obtains a convergent (i.e. Noetherian and confluent) reduction system.

The confluence proof (of which we have completed significant parts) appears to be rather lengthy and laborious, at least if one follows the well-known strategy of the Diamond Lemma [7]. Moreover, such a proof will have a much more computational flavour than the current article. Therefore we plan to present the confluence result in a separate publication.

\section{Conclusion}

As pointed out earlier, our construction of $\mathcal{G}\left[\int\right]$ is originally motivated from computational algebra: We want to build up algorithmic support for calculations with integral operators, substitutions and function expansions. In particular, we aim to represent, compute and manipulate (e.g. factor) Green's operators for suitable classes of LPDE boundary problems, as explained in the Introduction. This necessitates the following further tasks:

- Adjoining derivations to form partial integro-differential operators with linear substitutions.

- For both operator rings, prove confluence to ensure unique normal forms and hence deciding equality (relative to deciding equality in the given coefficient domain $\mathcal{G}$ ).

- Develop methods for computing and factoring LPDE Green's operators, as for example in [34].

- Study how the classical Green's function can be extracted from the Green's operator of certain LPDE boundary problems.

- Provide an implementation in a computer algebra package.

We plan to address these and related items in future work.

Apart from these rather obvious further developments, the material presented in this paper also contains some more intrinsic topics that might deserve a detailed investigation. Our notion of Rota-Baxter hierarchy $\left(\mathcal{F}, \int^{x_{n}}\right)_{n \in \mathbb{N}}$ is a first attempt to provide an algebraic framework to study integral operators and linear substitutions. But one could also try laying the foundations deeper, for example by using operads with a scaling action. The operad of Rota-Baxter algebras [2,3] has played a key role in understanding splitting of operads initiated by Loday [26]. In our case, note that the operation $f\left(x_{1}\right) \mapsto f\left(x_{1}+x_{2}\right)$ in the hierarchy $\mathcal{F}$ is similar to a coproduct except that it is of type $\mathcal{F}_{1} \rightarrow \mathcal{F}_{2}$ rather than $\mathcal{F}_{1} \rightarrow \mathcal{F}_{1} \otimes \mathcal{F}_{2}$. Working on multivariate functions $f\left(x_{1}, \ldots, x_{n}\right)$ and using similar 
substitutions

$$
\Delta_{i}:=\Delta_{i, n}: \quad x_{j} \mapsto x_{j}(j<i), \quad x_{i} \mapsto x_{i}+x_{i+1}, \quad x_{j} \mapsto x_{j+1}(j>i)
$$

one gets a family of operations $\Delta_{i, n}: \mathcal{F}_{n} \rightarrow \mathcal{F}_{n+1}$ generalizing the coproduct of a bialgebra. One may check that they satisfy the commutation rule $\Delta_{j} \Delta_{i}=\Delta_{i+1} \Delta_{j}$ for $i \geq j$. If one writes $\alpha: \mathcal{F}_{2} \rightarrow \mathcal{F}_{1}$ for the addition map, one has $\Delta_{i, n}(f)=f o_{n, 2, i} \alpha$ for $f \in \mathcal{F}_{n}$. Using the language of PROPs $[27,29,43]$, the commutation rule follows from the axioms and the associativity of $\alpha$. In a similar vein, we can express the scaling action by $d_{i, \lambda}^{*}(f)=f \circ_{n, 1, i} \lambda^{*}$ where $\lambda^{*}: \mathcal{F}_{1} \rightarrow \mathcal{F}_{1}$ is the substitution $x \mapsto \lambda x$. From the $\Delta_{i, n}$ and the $d_{i, \lambda}$ one can then build a Rota-Baxter hierarchy by decomposing matrices into permutation matrices, diagonal matrices and evaluations.

The advantage of this approach is that it would lead naturally to a generalization where $f \circ_{n, m, i} g$ is allowed for any $g \in \mathcal{F}_{m}$, not only for $g=\alpha$ and $g=\lambda^{*}$. For the Rota-Baxter operators we would then require the general substitution rule of multivariable calculus (and for the derivations the general chain rule), as it is satisfied for $C^{\infty}\left(\mathbb{R}^{\infty}\right)$.

Another possible generalization is more geometrically motivated: As mentioned earlier (Remark 2.6), for $C^{\infty}\left(\mathbb{R}^{\infty}\right)$ one can interpret our formulation of the chain rule as integration over certain parallelepipeds. However, this is not sufficient to provide a genuine theory of integration over simplices. In fact, it cannot: The definition of simplices requires some notion of orientation (e.g. coming from the order structure in the case $K=\mathbb{R}$ ) while we have only assumed algebras over a general field $K$. Nevertheless, it seems what we are missing is "not much" in a certain sense. Perhaps oriented sets [6, §2.6, Expl. 9b] could be used for endowing the vertices of a simplex with an orientation. On a more fundamental level, abstract simplicial complexes might provide just the right structure. In either case, the simplex would have one "variable vertex" (running along a fixed 1-cell), to ensure an indefinite integral and hence some kind of Rota-Baxter operator.

This in turn could be compared to the integration of differential forms over simplices, a species of definite integral that is crucial in de Rham cohomology (where $k$-chains are built from $k$-simplices). On the manifold $\mathbb{R}$, the connection between the Rota-Baxter operator of indefinite integration and the definite integration of differential forms is given by the Fundamental Theorem of Calculus. Roughly speaking, its generalization to higher dimensions is Stokes' Theorem. It would be interesting to explore this connection in the light of Rota-Baxter hierarchies.

\section{ACKNOWLEDGMENTS}

This work was supported by the National Science Foundation of US (Grant No. DMS 1001855), the Engineering and Physical Sciences Research Council of UK (Grant No. EP/I037474/1) and the National Natural Science Foundation of China (Grant No. 11371178).

\section{REFERENCES}

[1] H. Albrecher, C. Constantinescu, G. Pirsic, G. Regensburger, and M. Rosenkranz. An algebraic approach to the analysis of gerber-shiu functions. Insurance: Mathematics and Economics, Special Issue on Gerber-Shiu Functions:accpeted, 2009. 18 
[2] C. Bai, O. Bellier, L. Guo, and X. Ni. Splitting of operations, Manin products, and Rota-Baxter operators. Int. Math. Res. Not. IMRN, (3):485-524, 2013. 40

[3] C. Bai, L. Guo, and J. Pei. Splitting of operads and rota-baxter operators on operads. Preprint on arXiv:1306.3046., 2015. 40

[4] G. Baxter. An analytic problem whose solution follows from a simple algebraic identity. Pacific J. Math., 10:731-742, 1960. 2

[5] T. Becker and V. Weispfenning. Gröbner bases, volume 141 of Graduate Texts in Mathematics. Springer, New York, 1993. A computational approach to commutative algebra, In cooperation with Heinz Kredel. 15

[6] F. Bergeron, G. Labelle, and P. Leroux. Combinatorial species and tree-like structures, volume 67 of Encyclopedia of Mathematics and its Applications. Cambridge University Press, Cambridge, 1998. Translated from the 1994 French original by Margaret Readdy, With a foreword by Gian-Carlo Rota. 41

[7] G. M. Bergman. The diamond lemma for ring theory. Advances in Mathematics, 29(2):179-218, August 1978. 40

[8] B. Buchberger and M. Rosenkranz. Transforming problems from analysis to algebra: a case study in linear boundary problems. J. Symbolic Comput., 47(6):589-609, 2012. 9

[9] P. Cartier. A primer of Hopf algebras. In Frontiers in number theory, physics, and geometry. II, pages 537-615. Springer, Berlin, 2007. 19

[10] P. M. Cohn. Universal algebra, volume 6 of Mathematics and its Applications. D. Reidel Publishing Co., Dordrecht-Boston, Mass., second edition, 1981. 36

[11] A. Connes and D. Kreimer. Renormalization in quantum field theory and the Riemann-Hilbert problem. I. The Hopf algebra structure of graphs and the main theorem. Comm. Math. Phys., 210(1):249273, 2000. 2

[12] R. W. Downie. An introduction to the theory of quantum groups. Master's thesis, Eastern Washington University, June 2012. 19, 26

[13] D. G. Duffy. Green's functions with applications. Studies in Advanced Mathematics. Chapman \& Hall, Boca Raton, FL, 2001. 2

[14] L. C. Evans and R. F. Gariepy. Measure theory and fine properties of functions. Studies in Advanced Mathematics. CRC Press, Boca Raton, FL, 1992. 14

[15] U. Franz. Lévy processes on quantum groups and dual groups. In Quantum independent increment processes. II, volume 1866 of Lecture Notes in Math., pages 161-257. Springer, Berlin, 2006. 36

[16] G. Grätzer. Universal algebra. Springer, New York, second edition, 2008. With appendices by Grätzer, Bjarni Jónsson, Walter Taylor, Robert W. Quackenbush, Günter H. Wenzel, and Grätzer and W. A. Lampe. 36

[17] L. Guo. An Introduction to Rota-Baxter Algebras. International Press, 2012. 2, 9, 19, 23

[18] L. Guo and W. Keigher. Baxter algebras and shuffle products. Adv. Math., 150(1):117-149, 2000. 9

[19] L. Guo and Z. Lin. Representations and modules of rota-baxter algebras. Preprint, 2015. 30

[20] M. E. Hoffman. The algebra of multiple harmonic series. J. Algebra, 194(2):477-495, 1997. 2

[21] M. E. Hoffman. Algebraic aspects of multiple zeta values. In Zeta functions, topology and quantum physics, volume 14 of Dev. Math., pages 51-73. Springer, New York, 2005. 2

[22] T. W. Hungerford. The free product of algebras. Illinois J. Math., 12:312-324, 1968. 36

[23] N. Jacobson. Basic algebra. II. W. H. Freeman and Company, New York, second edition, 1989. 36

[24] R.-Q. Jian and J. Zhang. Rota-baxter coalgebras. Preprint, September 2014. 24

[25] E. Kolchin. Differential algebra and algebraic groups, volume 54 of Pure and Applied Mathematics. Academic Press, New York-London, 1973. 2

[26] J.-L. Loday. Dialgebras. In Dialgebras and related operads, volume 1763 of Lect. Notes in Math., pages 7-66. Springer, 2002. 40

[27] J.-L. Loday. Generalized bialgebras and triples of operads. Astérisque, 320:x+116, 2008. 41 
[28] M. Manetti. Differerential graded coalgebras. Lecture notes from a course on deformation theory held at UniRoma 1, 2012. 20, 22

[29] M. Markl. Operads and PROPs. In Handbook of algebra. Vol. 5, volume 5 of Handb. Algebr., pages 87-140. Elsevier/North-Holland, Amsterdam, 2008. 41

[30] G. Regensburger and M. Rosenkranz. An algebraic foundation for factoring linear boundary problems. Ann. Mat. Pura Appl. (4), 188(1):123-151, 2009. DOI:10.1007/s10231-008-0068-3. 3

[31] J. F. Ritt. Differential Equations from the Algebraic Standpoint. American Mathematical Society, New York, 1932. 2

[32] J. F. Ritt. Differential algebra. Dover Publications Inc., New York, 1966. 2

[33] M. Rosenkranz. A new symbolic method for solving linear two-point boundary value problems on the level of operators. J. Symbolic Comput., 39(2):171-199, 2005. 2

[34] M. Rosenkranz and N. Phisanbut. A symbolic approach to boundary problems for linear partial differential equations: Applications to the completely reducible case of the Cauchy problem with constant coeffcients. In $C A S C$, pages 301-314, 2013. 3, 4, 40

[35] M. Rosenkranz and G. Regensburger. Solving and factoring boundary problems for linear ordinary differential equations in differential algebras. Journal of Symbolic Computation, 43(8):515-544, 2008. $2,3,18$

[36] M. Rosenkranz, G. Regensburger, L. Tec, and B. Buchberger. A symbolic framework for operations on linear boundary problems. In V. P. Gerdt, E. W. Mayr, and E. H. Vorozhtsov, editors, Computer Algebra in Scientific Computing. Proceedings of the 11th International Workshop (CASC 2009), volume 5743 of LNCS, pages 269-283, Berlin, 2009. Springer. 4

[37] M. Rosenkranz, G. Regensburger, L. Tec, and B. Buchberger. Symbolic analysis of boundary problems: From rewriting to parametrized Gröbner bases. In U. Langer and P. Paule, editors, Numerical and Symbolic Scientific Computing: Progress and Prospects, pages 273-331. Springer, 2012. 2, 38

[38] G.-C. Rota. Baxter algebras and combinatorial identities (I, II). Bull. Amer. Math. Soc., 75:325-334, 1969. 2

[39] G.-C. Rota. Baxter operators, an introduction. In Gian-Carlo Rota on Combinatorics, Introductory papers and commentaries. Birkhäuser, Boston, 1995. 2

[40] I. Stakgold. Green's functions and boundary value problems. John Wiley \& Sons, New York, 1979. 2

[41] M. E. Sweedler. Hopf algebras. Mathematics Lecture Note Series. W. A. Benjamin, Inc., New York, 1969. 19

[42] T. Traynor. Change of variable for Hausdorff measure. Presented at the Workshop di Teoria della Misura e Analisi Reale, Grado, Italy, September/October 1993. 14

[43] B. Vallette. A Koszul duality for PROPs. Trans. Amer. Math. Soc., 359(10):4865-4943, 2007. 41

[44] W. T. Wu. A constructive theory of differential algebraic geometry based on works of J. F. Ritt with particular applications to mechanical theorem-proving of differential geometries. In Differential geometry and differential equations (Shanghai, 1985), volume 1255 of Lecture Notes in Math., pages 173-189. Springer, Berlin, 1987. 2

[45] D. Zagier. Values of zeta functions and their applications. In First European Congress of Mathematics, Vol. II (Paris, 1992), volume 120 of Progr. Math., pages 497-512. Birkhäuser, Basel, 1994. 2 
School of Mathematics, Statistics and Actuarial Science, University of Kent, CanterBURY CT2 7NF, ENGLAND

E-mail address: M.Rosenkranz@kent.ac.uk

School of Mathematics and Statistics, Key Laboratory of Applied Mathematics and Complex Systems, Lanzhou University, Lanzhou, Gansu, 730000, P.R. China

E-mail address: gaoxing@lzu.edu.cn

Department of Mathematics and Computer Science, Rutgers University, Newark, NJ 07102, US

E-mail address: liguo@rutgers.edu 